\title{
NROB2 regulation during Primary Sclerosing Cholangitis defines a metabolic and pre-malignant reprogramming of Cholangiocyte
}

\section{AUTHORS:}

Christophe Desterke, $\mathrm{PhD}^{1}$; Cyrille Feray, $\mathrm{MD}, \mathrm{PhD}^{2}$

\section{AFFILIATIONS:}

1: Centre Hépato-Biliaire, Hôpital Paul-Brousse, Assistance Publique-Hôpitaux de Paris, Université Paris-Saclay, unité Institut National de la Santé et de la Recherche Médicale UA9, Villejuif, France

2: Centre Hépato-Biliaire, Hôpital Paul-Brousse, Assistance Publique-Hôpitaux de Paris, Université Paris-Saclay, unité Institut National de la Santé et de la Recherche Médicale 1193, Villejuif, France

\section{SUMMARY:}

Primary Sclerosing Cholangitis (PSC) is an idiopathic, cholestatic liver disease that is characterized by persistent, progressive, biliary inflammation leading to cirrhosis. These patients present higher risk for developing bile duct cancers.

Biomedical text-mining related to PSC symptoms like: biliary inflammation, biliary fibrosis, biliary cholestasis was initiated to collect gene associations with this pathophysiology. The text mining work was integrated in distinct omics data such as human transcriptome of PSC liver, Farnesoid $\mathrm{X}$ receptor (FXR) functional liver transcriptome and liver single cell transcriptome of the Abcb4-/- model of PSC. A molecular network implicated in abnormal hepatobiliary system physiology was built and confirming a major implication of $\mathrm{Nr} 0 \mathrm{~b} 2$ and its associated nuclear receptors like FXR in a metabolic cascade that could influence immune response. TNFRSF12A/TWEAK receptor, was found up regulated in PSC liver independently of FXR regulation and TWEAK signaling is known for its implication in pre-conditioning niche of cholangiocarcinoma. NR0B2 deregulation in PSC liver was found independent of gender, age and body mass index surrogates. At single cell level, Nr0b2 up regulation was 
found in cholangiocytes but not in hepatocytes. In affected cholangiocytes, the cell trajectory built on NrOb2 expression, revealed implication of several metabolic pathways for detoxification like sulfur, glutathione derivative and monocarboxylic acid metabolisms. On this cell trajectory it was discovered some molecules potentially implicated in carcinogenesis like: GSTA3, ID2 and mainly TMEM45A a transmembrane molecule from golgi apparatus considered as oncogene in several cancers. All together, these observations found in humanPSC liver and in its murine models allowed to highlight an independent deregulation of NR0B2 with a metabolic and premalignant reprogramming of cholangiocytes.

KEYWORDS: cholangiocyte, primary sclerosing cholangitis, metabolism, Farnesoid X receptor, transcriptome, Small Heterodimer Partner, pre-malignancy, TMEM45A

\section{INTRODUCTION}

Primary sclerosing cholangitis is a progressive, cholestatic hepatic disease of unknown etiology. It is characterized by progressive inflammation, destruction, and fibrosis of the intrahepatic and extrahepatic bile ducts (Lazaridis \& LaRusso, 2016). PSC is a rare disease with an incidence that ranges from 0 to 1.3 cases per 100,000 persons per year (Molodecky et al., 2011). Co-occurrence of inflammatory bowel disease (IBD), most commonly ulcerative colitis (UC), has been reported in up to $80 \%$ of patients with PSC (Mertz, Nguyen, Katsanos, \& Kwok, 2019). The close association between PSC and IBD indicates the involvement of the gut-liver axis. Also, environmental factor like microbiota has emerged as one of the most important potential environmental players in chronic inflammatory diseases like PSC (Rühlemann et al., 2019). Enterohepatic circulation referred to the metabolite exchange between liver and intestine may play an important aspect of the PSC pathophysiology because metabolome of PSC portal blood and bile samples signed a peculiar molecular profile as compared to ones of disease and non-disease controls (Tietz-Bogert et al., 2018, p.).

PSC causes multifocal strictures of the biliary tree, biliary infections, and accumulation of toxic bile acids, which results in an increased expression of adhesion and antigen-presenting molecules and inflammatory mediators. These observations are a good field for emergence of carcinogenesis that could affect biliary tracts (Chalasani et al., 2000; Rizvi, Eaton, \& Gores, 2015). There is no effective treatment which can delay its progression or ameliorate the 
transplant-free survival. More recently, novel pharmacological agents emerged aiming at: modulation of bile composition, immunomodulation, targeting the gut microbiome, targeting fibrosis (Floreani \& De Martin, 2021).

Many aspects of the pathogenesis of PSC remain unclear. Biomedical literature is traditionally used as a way to inform scientists of the relevance of genes in relation to a research topic (Fleuren \& Alkema, 2015). Previously, test of distinct biomedical text mining web tools to integrate gene relations with omics data highlighted 'Génie' algorithm (Fontaine, Priller, Barbosa-Silva, \& Andrade-Navarro, 2011) as a robust and sensitive machine learning tool to rank best literature associated genes. By textmining (Génie) integration in single cell transcriptome it could be possible to highlighted podocytes markers implicated in Focal Segmental Glomeral Sclerosis (Desterke, Lorenzo, \& Candelier, 2021). 'Génie' algorithm also contributed to understand lipidome deregulations during nonalcoholic fatty liver disease (Pirola \& Sookoian, 2021). 'Génie' is a powerful and fast tool that prioritizes the whole gene sets of hundreds of species for any biomedical topic, taking advantage of annotations-linking gene and bibliography (Fontaine et al., 2011). In this work, biomedical text-mining related to PSC symptoms allowed to enrich a gene set of molecules that was used originally to analyze different omics data coming from human PSC liver biopsies and distinct animal models of PSC. Independent deregulation of NR0B2 in PSC allowed to highlight a metabolic and premalignant reprogramming of the affected cholangiocytes.

\section{MATERIAL AND METHODS}

\section{Biomedical Pubmed textmining}

'Génie' Data mining tools performed gene prioritization (Fontaine et al., 2011) by linking scientific litterature from MEDLINE database with gene information from NCBI Gene database and orthologue gene information from different species with HomoloGene database (E. Sayers, 2010; E. W. Sayers et al., 2009). Génie algorithm works by connecting gene identifier and gene orthologue NCBI databases with genes related to input keywords obtained by building a textmining classifier with article abstracts from PUBMED literature ressource. Primary Sclerosing Cholangitis related symptom Medical Subject Headings (MeSH) terms were individually submitted to 'Génie' algorithm on 2021-july-2nd. All abstract of PUBMED 
were taking as background for the analysis of genes corresponding to Homo sapiens coding proteins. All orthologue information were collected during the process, p-value cutoff for abstract selection was fixed to $\mathrm{p}<0.01$ and False Discovery Rate cutoff for gene selection was fixed to $\mathrm{p}<0.01$. This last step of FDR adjustment allowed to correct false positive discovery rate and so to minize the influence of one pecular article in the globality of the analysis of gene enrichment.

\section{Public datasets}

\section{Transcriptome studies}

1/ Human liver biopsies ( $\mathrm{n}=134)$, GSE61256 (Horvath et al., 2014): transcriptome experiments performed on human biopsies were downloaded and annotated with the corresponding annotation platform: GPL11532 used for technology version DNAChipAffymetrix Human Gene 1.1 ST Array (alias HuGene-1_1-st) available at the fellowing address: https://www.ncbi.nlm.nih.gov/geo/query/acc.cgi?acc=GPL11532 (accessed on 2021, july $\left.2^{\text {nd }}\right)$.

2/ Mus musculus liver FXR functional study GSE54557 (Zhan et al., 2014): C57BL/6J Mus musculus liver samples of 10-16 weeks. Transcriptome of liver samples in presence or not of liver FXR knock-out and with or without treatment with GW4064 FXR agonist experience en triplicate. Transcriptome matrix was downloaded and annotated with the annotated platform GPL8321 corresponding to the DNAchip technology: Affymetrix Mouse Genome 430A 2.0 Array (alias Mouse430A_2) available at the following address: https://www.ncbi.nlm.nih.gov/geo/query/acc.cgi?acc=GPL8321 (accessed on 2021, july 2nd).

\section{Single cell transcriptome study}

Mus musculus Abcb4-/- single cell transcriptome liver samples GSE168758 (Reich et al., 2021): Single cell transcriptome libraries of liver cells were prepared with Chromium Single Cell 3' NextGEM Reagent Kit v3.1 (10X Genomics) to performed sequencing on NextSeq 550 (Illumina) and demultiplexing pipeline with CellRanger v5.0.0 (10x Genomics). MTX matrix file format were downloaded at the following address: https://www.ncbi.nlm.nih.gov/geo/query/acc.cgi?acc=GSM5165876 (accessed on 2021, july 2nd). 


\section{Bioinformatics analyses}

Bioinformatics analysis were performed in $\mathrm{R}$ software environment version 4.1.0 under Ubuntu version 20.04 LTS. Graphical representation were performed with ggplot2 graph definition implemented in ggplot2 R-package version 3.3.5 (Wickham, 2009, p. 2).

\section{Transcriptome Analyses}

Unsupervized analysis performed by principal component analysis used FactoMineR Rpackage version 2.4 (Lê, Josse, \& Husson, 2008). Gene Functional enrichment analyses were performed with Toppgene web application (J. Chen, Bardes, Aronow, \& Jegga, 2009). Differential expressed genes were identified with LInear Model from Microarray (LIMMA) algorithm implemented in R Bioconductor package version 3.44.3 (Ritchie et al., 2015). FXR regulation dependency analysis with Pavlidis Template Matching algorithm with retaining significant genes on absolute value of correlation coefficient and with a cutoff p-value less than 0.05 (Pavlidis \& Noble, 2001). Expression heatmap were drawn with pheatmap Rpackage version 1.012 .

\section{Single cell transcriptome analysis}

Single transcriptome analyses at whole liver level were performed with Seurat4 R-package version 4.0.3 (Butler, Hoffman, Smibert, Papalexi, \& Satija, 2018). Briefly, after canonical correlation, filtration (cells expressing minimally 300 genes) and scaling between replicated samples $(\mathrm{n}=4)$ of wildtype and Abcb4-/- groups a normalized single cell object comprising 46.087 transcriptomes was built. Dimension reduction was carried out by principal component analysis on most variable features (50 components) and secondly: t-distributed stochastic neighbor embedding (t-SNE) and Uniform Manifold Approximation and Projection (UMAP) dimensionality reduction was performed on the 30 best components of the PCA. Cell cluster communities were detected graph-based clustering approaches (Xu \& Su, 2015). Abcb-/cholangiocytes identified in clusters $0-5-11$ were select were randomly down sampled to 1/10e of their initial amount to build a matrix finally containing 1316 cells. Single cell trajectory performed on Abcb4-/- cholangiocyte with monocle2 R Bioconductor package version 2.20.0 (Trapnell et al., 2014). Cell hierarchy was construct on alternative expression of Sox 9 and NrOb2 in Abcb4-/- cholangiocytes. Pseudotime transformation on cell trajectory 
was performed with DDRtree algorithm and pseudotime cluster identification was done by pseudotime expression heatmap drawn with best genes found significant

\section{Network analyses}

Gene and function relations identified were collected with functional enrichment and network were built with Cytoscape standalone software version 3.6.0 (Cline et al., 2007) for their visualization. Protein-protein interaction (PPI) network were performed with NetworkAnalyst (Xia, Gill, \& Hancock, 2015) webtools based on interaction network databases: STRING (Szklarczyk et al., 2017) or liver specific DifferentialNet. Functional inference was done on PPI networks with Gene Ontology Biological Process (GO-BP) database (The Gene Ontology Consortium, 2017).

\section{RESULTS}

\section{Biomedical text mining for discovery of gene relations with symptoms of primary} sclerosing cholangitis

Classically, the litterature dealing with genes, as stored in the MEDLINE database (E. W. Sayers et al., 2009) of biomedical references has been used to do gene prioritization. Many methods for gene prioritization using litterature data are based on co-occurence analysis of keywords and extract gene names from abstracts (Krallinger, Valencia, \& Hirschman, 2008). 'Génie' algorithm is based on Naive bayes classifier and take advantage of linking orthologues genes study in many different species (Fontaine et al., 2011). Primary Sclerosing Cholangitis (PSC) is an Idiopathic, Heterogeneous, cholestatic liver disease that is characterized by persistent, progressive, biliary inflammation and fibrosis (Lazaridis \& LaRusso, 2016). Three related MESH terms for indirect characterization of PSC symptomatology were used to process in 'Génie' algorithm and trigger gene related to the disease with the following exact terms: 'biliary inflammation', 'biliary fibrosis', 'biliary stasis'. These respective analyses allowed to collect a common set of 525 ranked genes (workflow, Figure 1 and Supplemental Table 1). NR1H4 alias FXR Farnesoid X receptor was found as the best ranked gene at cross of these three independent text mining analyses 
(Supplemental Table 1). This text mining results allowed to give us a restrictive gene list (525) to investigated downstream omics analyses in context of PSC disorder as it is highlight graphically on the workflow of the article (Figure 1). Starting omics analyses with reduced size gene sets allowed to drastically eliminate background noise and False Discovery Rate in these experiments as it is shown with Gene Set Enrichment Analysis (GSEA) procedure (Subramanian et al., 2005).

\section{Abnormalities in physiology of hepatobiliary system during primary sclerosing cholangitis}

Primary sclerosing cholangitis (PSC) is a chronic hepatic syndrome with fibro-inflammatory affection of the bile ducts. In order to test our txt mining PSC symptom related gene set, a bioinformatics investigation was performed on transcriptome study GSE61256 (Figure 1) (Horvath et al., 2014) which is composed of liver biopsy samples from PSC $(n=14)$ patients as compared to distinct control groups such as: PBC $(n=11)$, normal control $(n=38)$, normal obese $(n=24)$, NAFLD $(n=23)$, NASH $(n=24)$. Data from GSE61256 were reduced in dimension with the 525 PSC symptom related genes but only 507 of them were find provided in these type of experiments. Unsupervised principal component analysis revealed an individual stratification of PSC samples as compared to every other control groups and especially as compared to PBC control which could harbored also these three phenotypes (pvalue=6.89E-4, Figure 2A). With less importance, these genes also discriminates PBC samples from other liver control groups in the second map of PCA analysis (Supplemental Figure 1). These results suggested that in its entirety PSC symptom related gene set obtained by text mining seems to be specific to predict liver sample transcriptome of PSC as compared to divers control groups. Effectively, starting from these 507 molecules supervised 'LInear Model from Microarray' (LIMMA) algorithm for differential expression analysis allowed to characterized a significant expression profile of PSC liver samples as compared to all other pooled control groups (Figure 2B and Supplemental Table 2). Among the best up regulated genes, FOXP3 was found the best one followed by NR0B2, TNFRSF1A and FGF19. A distinct trait of expression of up regulated for PSC samples could be observed on the right of expression heatmap performed with up regulated genes (Figure 2C). Functional enrichment performed with these PSC up regulated genes on Mouse Phenotype database allowed to characterized were found mainly implicated in abnormal liver physiology and abnormal 
hepatobiliary system physiology (Figure 2D). As PSC is a hepatic disorder affecting bile ducts a functional enrichment network was built on affection of hepatobiliary system. On this hepatobiliary system phenotype network we could observed that some of the top up regulated genes in PSC are present such as: FOXP3, TNFRSF12A, TNFRSF1A, LCN2 and NR0B2 (Figure 2E). Starting from PSC symptom related gene found by text mining we could predict with liver samples, a PSC specific up regulated network implicated in hepatobiliary system phenotype.

\section{Hepatic regulation of immune response and intracellular receptor signaling under FXR dependency}

In PSC symptom related genes found by text-mining we could observed that NR1H4 alias Farnesyl X receptor (FXR) was the best ranked gene associated to the literature in relations with input symptom MeSH. FXR is a nuclear receptor implicated in bile acide metabolism and have been widely study in hepatocyte biology such as whole gene ChIP-sequencing experiments (Zhan et al., 2014). Liver transcriptome study were also performed on hepatic functional studies targeting FXR: such as GSE54557 dataset which investigate liver samples of Mus musculus with or without liver FXR knock-out reversed of not by GW4064 agonist treatment (Zhan et al., 2014). Data from GSE61256 were reduced in dimension with the 525 PSC symptom related genes and Pavlidis Template Matching (PTM) algorithm was used to understand FXR regulation dependency: FXR liver knock-out reversed by GW4064 FXR agonist (Figure 3A and Supplemental Table 3): 91 genes were found significantly regulated through FXR dependency in liver samples. These significant genes allowed to significantly stratified experimental samples of FXR liver KO from wildtype ones (WT) and reversion by FXR agonist was obtained only in wildtype samples (p-value=1.66.E-4, Figure 3B). Molecules with positive regulation following FXR liver dependency were selected to draw an expression heatmap (Figure 3C) which implicated a protein-protein interaction (PPI) network mainly implicated in regulation of immune response (nodes in green, Figure 3D) and in intracellular receptor mediated signaling pathway (in blue, Figure 3D). Concerning intracellular receptor signaling, three main blue crosstalk could be observed on the PPI network (Figure 3D): NR1H4, NR1I2 and PPARGC1A which are closely connected. Concerning immune response we could observed (Figure 3C) a liver FXR regulation dependency of CD274 alias PDL1 immune checkpoint. These results suggest that starting 
from PSC symptom related gene list we could predict a hepatic expression profile under FXR regulation dependency and so confirming its potential functional importance in the pathology.

\section{Nrb02 is the best predictive marker regulated in liver of primary sclerosing cholangitis under FXR dependency}

In order to evaluate FXR dependency in PSC related human liver expression profile that we identified we investigated to cross the human study signature (Supplemental Table 2 and Figure 2) with liver FXR functional signature (Supplemental Table 3 and Figure 3). This investigation allowed to highlight 34 common regulated molecules between the two studies (Figure 4A). Proceeding supervised machine learning by leave one out cross validation algorithm (pamr) between human liver PSC samples and other liver control samples (GSE61256), it could possible to defined a predictive molecular ranking (Supplemental Table 2) with a minimal misclassification error of 8.9 percent between samples (Figure 4B). Inside these best molecular ranking to predict human PSC liver we could observed some molecules with an FXR dependent regulation (in pink, Figure 4C), such as: NR0B2, CYP7A1, DGAT2, NR1H4 (alias FXR), TNFRSF1A, BCHE, KLF6 and CEBPA. NR0B2 at the top of PSC prediction with FXR dependency was investigated by univariate analysis stratified on gender of the patients. Effectively, NR0B2 was found up regulated in PSC samples and more sensitively in samples of female gender (Figure 4D). In a biological and clinical multivariate model, we could observed that up regulation of NR0B2 was significantly up regulated in PSC liver samples but independently of the gender, the age and body mass index (BMI) of the patients. These results suggest that the nuclear receptor NR0B2 is the best predictive marker up regulated in PSC liver which harbored an FXR dependent regulation is its regulation is independent of the age, gender and BMI of the patients.

\section{Up regulation of $\mathrm{Nrb02}$ in cholangiocyte compartment of Abcb4-/- liver}

Abcb4-/- (Mdr2) mice are widely used as model for sclerosing cholangitis because it recapitulates the progressive fibrosing cholangitis aspect of human PSC and also develop intrahepatic malignancy (Fickert et al., 2004, 2014). Single cell transcriptome performed in liver of wild type and Abcb4-/- mice models have been provided in GSE168758 dataset (Reich et al., 2021, p. 5). With 4 replicates of liver in groups WT and Abcb4-/- a single cell normalized object was built with 46.087 cells which individually expressed a minimum of 
300 genes. After dimension reduction and clustering 15 cell communities were identified and classed in five main cell types: hepatocytes, cholangiocytes, endothelial cells, macrophages and lymphocytes (Figure 5A and Supplemental Figure 2). Wild type livers harbored more hepatocytes as compared Abcb4-/- livers (in red, Figure 5B) and Abcb4 livers harbored more cholangiocytes than wild type livers (in blue, Figure 5B). Sox9 is a transcription factor implicated in the biliary development (Antoniou et al., 2009). Epcam and Sox9 expressions follow CK19 expression during human intrahepatic biliary tree formation through several developmental stages involving an initial transition of primitive hepatocytes into cholangiocytes (Vestentoft et al., 2011). Sox9 was found over expressed in Abcb4-/- livers as compared to wild type ones (Figure 5C) and especially in cholangiocyte compartment (Figure 5D) which correspond to cell clusters : 0, 5 and 11 (Supplemental Figure 3A). Similarly, an overexpression of $\mathrm{NrOb} 2$ was found in Abcb4-/- livers as compared to wild type ones (Figure 5E). Expression of $\mathrm{NrOb} 2$ was found detected in hepatocyte and cholangiocyte cell compartment in both liver genotypes (Figure 5F). Overexpression of $\mathrm{NrOb} 2$ in liver Abcb4-/- livers concerned cell clusters 0, 5 and 11 (Figure 5G and Supplemental Figure 3B) which were confirmed as expressing Sox9 and Epcam cholangicoytes markers (Supplemental Figure 3A and 3C). Absence of $\mathrm{Nr} 0 \mathrm{~b} 2$ regulation was observed in hepatocyte compartment of Abcb4-/- (cluster 1, Figure 5G). These results suggest that Nr0b2 nuclear receptor was found up regulated in cholangiocyte compartment of the Abcb4-/- liver: mice model of sclerosing cholangitis.

\section{Metabolic regulation during cell trajectory of Abcb4-/- cholangiocytes}

$\mathrm{NrOb} 2$ alias small heterodimer partner is a nuclear receptor identified as a key transcription regulator affecting divers functions implicated in metabolism such as: bile acid metabolism, lipid metabolism, glucose and energy homeostasis (Zhang, Hagedorn, \& Wang, 2011, p. 2010). NrOb2 was found as best predictive marker of PSC in liver with FXR regulation dependency but its overexpression in Abcb4-/- mice model affect cholangiocyte but not hepatocyte cell compartment. Starting from single cell transcriptome of GSE168758 dataset, a random down sampling subset of Abcb4-/- cholangiocytes was selected to build a single cell trajectory based on alternative expression of $\mathrm{NrOb} 2$ and Sox9. With an affected cholangiocyte compartment comprising in total 1316 cells, 238 cells were characterized as Sox9 Nr0b2 double negative, 51 cells were characterized as negative for Sox 9 and positive for $\mathrm{Nr} 0 \mathrm{~b} 2,406$ 
cells were characterized as positive for Sox 9 and negative for $\mathrm{Nr} 0 \mathrm{~b} 2$, and 621 cells were characterized as Sox9 and Nr0b2 double positive (Figure 6A). The alternative expression of Sox9 and NrOb2 reflect a cell heterogeneity in Abcb4-/- the cluster heterogeneity of cholangiocytes clusters 0, 5 and found previously found at whole liver level (Supplemental Figure 4A and 4B). Pseudotime transformation of single cell transcriptome in this affected compartment (Figure 6 and Supplemental Figure 4C) reflect a cell trajectory with progressive modification of Sox9 (Supplemental Figure 4D) and Nr0b2 (Supplemental Figure 4E) expressions. Identification of most significant regulated genes on this cell trajectory (Supplemental Table 4) allowed to identified three distinct regulated gene clusters (Figure 6C). Expressions of Sox 9 and $\mathrm{Nr} 0 \mathrm{~b} 2$ were found closely regulated on the trajectory of the pseudotime green gene cluster (Figure 6C). Among the pseudotime green cluster, expression of Alb, Gsta3, Id2 and Tmem45a were found closely regulated with $\mathrm{Nr} 0 \mathrm{~b} 2$ and Sox9, especially Gsta3 very near Nr0b2 pseudotime regulation (Figure 6C and Supplemental Figure 4F). Protein-protein network performed with genes belonging to green pseudotime gene cluster with functional inference of Gene Ontology Biological Process database allowed to identified a main functional enrichment of positive regulation of metabolism (False Discovery Rate p-value=5.2E-23, Figure 6D). This metabolic functional enrichment allowed to mainly implicated sulfur compound metabolism, glutathione derivative biosynthesis, cellular detoxification and monocarboxylic acid metabolism (Figure 6E) which share some main regulated molecules (Figure 6F). These results suggest that heterogeneity of Nr0b2 expression inside Abcb4-/- cholangiocyte affected compartment defined a cell trajectory implicating an important regulation of metabolism with detoxification process.

\section{DISCUSSION}

Primary sclerosing cholangitis is a chronic, fibro-inflammatory disorder affecting intra/extrahepatic bile ducts without precise etiology and efficient therapy. The resulting cholestasis may progess to cirrhosis and liver failure (Karlsen, Folseraas, Thorburn, \& Vesterhus, 2017). Enterohepatic circulation have been proposed to participate to the pathophysiology of this disease because it could be diagnose a preponderance of inflammatory bowel disease in PSC patients (Boonstra et al., 2012) and also it have been discovery that the gut microbiota of PSC patients is distinct from those inflammatory bowel disease patients and healthy controls (Sabino et al., 2016). Progress remains to be made in the understanding of the physiopathology of this disease in order to elucidate why some of these 
patients have a higher risk of developing cholangiocarcinomas (Rizvi et al., 2015). Few liver transcriptome experiments were available in PSC disease (Horvath et al., 2014): experiments in which it might be interesting to observe cellular mechanisms consecutives to the symptoms of PSC disorder. The integration of biomedical text mining into high-dimensional omics data such as transcriptome data drastically reduces the error of positive false discovery (Desterke et al., 2021). With the aim of increasing the authenticity of discovery of these mechanisms in human liver transcriptome samples from PSC, a biomedical text-mining pipeline has been developed based on a triad of symptoms characterizing PSC: biliary inflammation, biliary fibrosis and biliary stasis (Lazaridis \& LaRusso, 2016). The intersection of these text-mining researches have made it possible to link 525 genes to the symptomatic of PSC disorder. The best literature associated gene by text-mining was NR1H4 alias FXR Farnesoid X receptor a nuclear receptor implicated in bile acid metabolism. Nuclear receptors (NRs) are a unique family of transcription factors (TFs) that are being recognized as key regulators of multiple functions in almost all aspects of mammalian development, metabolism and physiology (Gronemeyer, Gustafsson, \& Laudet, 2004). FXR is highly expressed in liver and is used as bile acid sensor by regulating genes that are critically involved in bile acid homeostasis, including bile acid biosynthesis, conjugation, and entero-hepatic circulation (Sinal et al., 2000). PSC symptom related genes found by biomedical text mining were used to analyzed human liver transcriptome and predicted a stratification of PSC samples as compared to samples from different controls: healthy normal liver, obese healthy donors, NAFLD, NASH and PBC. A PSC expression profile was able to be identified by differential expression analysis which highlighted important over expression of distinct molecules. FOXP3 was found as the best overexpressed gene in liver of PSC patients. FOXP3 transcription factor characterized the phenotype of regulatory T-lymphocyte immune cell sub population (Sakaguchi, Miyara, Costantino, \& Hafler, 2010) and bile acid metabolites have been shown to be implicated in the control regulatory T-lymphocyte cell differentiation (Hang et al., 2019). In genome of PSC patients, polymorphisms found in IL2RA gene affected FOXP3+ regulatory T-cell subpopulation of these patients (Sebode et al., 2014). Among best up regulated genes found in PSC it was found NR0B2 alias small heterodimer partner (SHP). NR0B2 has been identified as key transcription regulator implicated in divers biological function such as: bile acids; cholesterol and lipid metabolism, glucose and energy homeostasis (Zhang et al., 2011). NR0B2 alias Small heterodimer partner (SHP) is a unique nuclear receptor (NR) distinct from other conventional NRs in both its structure and function. The presence of a putative ligand-binding domain (LBD) classifies SHP as a member of the NR 
family, although the endogenous ligand has not been identified (Seol, Choi, \& Moore, 1996) and NR0B2 is known as common heterodimer partner of FXR implicated in bile acid metabolism (Goodwin et al., 2000). Among best up-regulated genes found in PSC liver, FGF19 is implicate in bile acid homeostasis and effectively it is a target of bile acids via FXR in enterocyte and recirculate in portal blood to bind to FGFR4 receptor on hepatocyte (Inagaki et al., 2005). Among best up regulated molecules in liver of PSC, it was found TNFRSF12A which is a receptor from TNF superfamily. TNFRSF12A is also named TWEAK receptor and TWEAK/Fn14 signaling have been associated to promote cholangiocarcinoma niche formation and progression (Dwyer et al., 2021). Through liver functional analysis, FXR regulation dependency was used to build a liver protein-protein interaction network which mainly implicated immune response and nuclear receptor signalling. Through immune response functionality, CD274 (alias PDL1) was found regulated under liver FXR regulation. Effectively, some clinical trials in cancer therapy under penbrolizumab (anti-PDL1) highlighted risk to developed sclerosing cholangitis (Tahboub Amawi, Tremaine, \& Venkatesh, 2020). In next step, human PSC gene signature was investigated with the FXR regulation dependency. NR0B2 was found as the best PSC predictive up regulated gene under FXR regulation and this relation is well known to be implicated in regulatory cascade of bile acid metabolism drive by nuclear receptors which repressed CYP7A1. CYP7A1 catalyzes the rate limiting step in bile acid biosynthesis. NR0B2 is known to repress CYP7A1 via activity inhibition of liver receptor homolog 1 (LRH-1) (Goodwin et al., 2000). In liver from PSC, CYP7A1 was confirmed to be down regulated as compared to liver samples from controls. Among best PSC predictive genes under FXR dependency, DGAT2, Diacylglycerol OAcyltransferase 2 (Brandt, McFie, \& Stone, 2016) implicated in biosynthesis of triglycerides was found repressed in PSC liver and effectively portal blood and bile lipidomics of PSC patients was found peculiar as compared to similar samples from disease controls and nondisease controls especially with increase of free fatty acid detection (Tietz-Bogert et al., 2018). NR0B2 alias SHP was found as the best predictive marker of PSC under FXR regulation dependency and its up regulation in PSC liver was found independent of the age, the gender and Body Mass Index (BMI) of the patients. Mdr2-/- alias Abcb4-/- mice model is widely used as a model of sclerosing cholangitis. It recapitulates the progressive fibrosing cholangitis aspect of human PSC. At single cell level in liver of Abcb4-/- mice, expression of $\mathrm{Nr} 0 \mathrm{~b} 2$ was detected in hepatocyte and cholangiocytes cell compartments but it's over expression as compared to wide type cells was observed only the cholangiocytes which highly expressed Sox9. Based on alternative expression of Sox9 and $\mathrm{Nr} 0 \mathrm{~b} 2$, single cell trajectory was 
built with in Abcb4-/- cholangiocyte cell compartment. Affected cholangiocytes highlighted a cell trajectory cluster comprising a network of molecules implicated in metabolism: sulfur component metabolism, glutathione derivative biosynthesis and monocarboxylic acid metabolism. N-acetylcysteine therapeutical option have been already tested in PSC, by its antimucolytic action it potentially reduce bile viscosity and improved clinical and laboratory parameters of PSC patients in a small study (Ozdil, Cosar, Akkiz, Sandikci, \& Kece, 2011). On NrOb2 cell trajectory of affected cholangiocyte, Gsta3 Glutathione S transferase was found with similar pseudotime expression meaning their closed regulation during cell trajectory of this affected cell compartment. Glutathione S-transferases (GST) like GSTA3 acts as antioxidant enzymes mainly in liver tissue through glutathione peroxidase activity toward phospholipid hydroperoxides (Yang, Sharma, Zimniak, \& Awasthi, 2002). GSTA3 belongs to the GST $\alpha$-class, which converts lipid peroxides to glutathione conjugates (Johansson \& Mannervik, 2001, p. 3-3). It has been shown that glutathione S-transferase A3 have a role in inhibiting hepatic stellate cell activation and rat hepatic fibrosis (H. Chen et al., 2019). Also, glutathione S-transferase A3 knockout mice harbores liver injury, oval cell proliferation and cholangiocarcinogenesis in (Crawford et al., 2017). Inhibitor of DNA binding 2 (ID2) was found also closely regulated on $\mathrm{NrOb} 2$ cell trajectory of Abcb4-/- cholangiocytes. ID2 is a member basic Helix Loop Helix transcription factor family. ID2 like other ID proteins lacks DNA binding domain and so transcription of downstream cell cycle targets like p16, p21(Benezra, Davis, Lockshon, Turner, \& Weintraub, 1990). ID2 like ID1 and ID3 proteins is frequently overexpressed in all biliary tract cancer (BTC) subtypes. Also, ID protein expression is deregulated in many tumors including pancreatic cancer a malignancy somehow related to bile tract cancers. During bile tract cancer, for subgroup of patients who had received chemotherapy, if nuclear expression of ID2 is negative these patients presented a better overall survival as compared those who were nuclear positive (Harder et al., 2013). With closed pseudotime expression on NrOb2 cell trajectory of Abcb4-/- cholangiocyte cell compartment, it was found the membrane molecule Tmem45a. Tmem45a is transmembrane molecule of 275 amino acids, predicted to have five to seven transmembrane domains and localized in the trans Golgi apparatus. This protein is over expressed in many cancers: breast cancer, liver cancer, renal cancer, glioma, head and neck cancer, ductal cancer and ovarian cancer: it could be supposed to act as an oncogene (Flamant et al., 2012; Guo et al., 2015; Lee et al., 2012; Manawapat-Klopfer et al., 2016; Sun et al., 2015; Wrzesiński et al., 2015). During breast and cervical tumors, there was found a higher level of expression for TMEM45A suggesting this molecule could be a biomarker of aggressiveness in these 
malignant pathologies (Manawapat-Klopfer et al., 2016). TMEM45A have been implicated in the proliferation, migration and invasion of glioma cancer cells (Guo et al., 2015; Sun et al., 2015). TMEM45A also confers chemoresistance in hypoxia condition for the cell death induced chemotherapeutic agents of breast cancer (Flamant et al., 2012).

\section{Conclusion:}

In this work we performed integration of biomedical text-mining related to PSC symptom in omics data such as human transcriptome of PSC liver, FXR functional liver transcriptome and liver single cell transcriptome of the Abcb4-/- model of PSC. This integrative work allowed to confirmed major implication of $\mathrm{NrOb} 2$ and its associated nuclear receptors like FXR in a metabolic cascade that could influence immune response. TNFRSF12A/TWEAK receptor, was found up regulated in PSC liver independently of FXR regulation and TWEAK signaling is known for its implication in pre-conditioning niche of cholangiocarcinoma. At single cell level, Nr0b2 up regulation was found in cholangiocytes but not in hepatocytes. In this affected cell compartment cell trajectory defined on Nr0b2 expression, highlighted several metabolic pathways of detoxification like sulfur, glutathione derivative and monocarboxylic acid metabolisms. On this cell trajectory it was discovered some molecules potentially implicated in carcinogenesis like: GSTA3, ID2 and mainly TMEM45A a transmembrane molecule considered as oncogene in several cancers. 


\section{DATA AVAILABILITY}

$\mathrm{R}$ bioinformatics code for single cell analyses performed with Seurat 4 and Monocle 2 packages on dataset GSE168758 are accessible on internet at the following address: www.github.com/cdesterke/pscsc (accessed on 2021, July $10^{\text {th }}$ ).

\section{ACKNOWLEDGMENTS}

Thanks to department 'Bloc OPératoire Augmenté' (BOPA) for access to their facilities.

\section{CONTRIBUIONS}

$\mathrm{CD}$ and $\mathrm{CF}$ : design of the experience, $\mathrm{CD}$ and $\mathrm{CF}$ : written manuscript, $\mathrm{CD}:$ data analysis, $\mathrm{CF}$ : suppervisor and correction of the manuscript 


\section{ABBREVIETIONS}

Abcb4: ATP Binding Cassette Subfamily B Member 4

BMI: body mass index

BTC: biliary tract cancer

DEGs: differential expressed genes

FDR: False Discovery Rate

FXR: Farnesoid X Receptor, alias NR1H4

GO-BP: Gene Ontology Biological Process database

GSEA: Gene Set Enrichment Analysis

GW4064: Farnesoid X Receptor agonist

ID2: inhibitor of DNA binding 2

KO: knock-out

LIMMA: LInear Model from Microarray

MeSH: Medical Subject Headings

NAFLD: Nonalcoholic fatty liver disease

NASH: Nonalcoholic steatohepatitis

NR: Nuclear receptor

NrOb2: Nuclear Receptor Subfamily 0 Group B Member 2, alias SHP: Small Heterodimer Partner

PBC: primary biliary cholangitis

PPI: Protein-protein interaction

PSC: primary sclerosing cholangitis

PTM: Pavlidis Template Matching

t-SNE: t-distributed stochastic neighbor embedding

UMAP: Uniform Manifold Approximation and Projection 


\section{REFERENCES}

Antoniou, A., Raynaud, P., Cordi, S., Zong, Y., Tronche, F., Stanger, B. Z., ... Lemaigre, F. P. (2009). Intrahepatic bile ducts develop according to a new mode of tubulogenesis regulated by the transcription factor SOX9. Gastroenterology, 136(7), 2325-2333. doi:

10.1053/j.gastro.2009.02.051

Benezra, R., Davis, R. L., Lockshon, D., Turner, D. L., \& Weintraub, H. (1990). The protein Id : A negative regulator of helix-loop-helix DNA binding proteins. Cell, 61(1), 49-59. doi: 10.1016/0092-8674(90)90214-y

Boonstra, K., van Erpecum, K. J., van Nieuwkerk, K. M. J., Drenth, J. P. H., Poen, A. C., Witteman, B. J. M., ... Ponsioen, C. Y. (2012). Primary sclerosing cholangitis is associated with a distinct phenotype of inflammatory bowel disease. Inflammatory Bowel Diseases, 18(12), 2270-2276. doi: $10.1002 /$ ibd.22938

Brandt, C., McFie, P. J., \& Stone, S. J. (2016). Biochemical characterization of human acyl coenzyme A : 2-monoacylglycerol acyltransferase-3 (MGAT3). Biochemical and Biophysical Research Communications, 475(3), 264-270. doi: 10.1016/j.bbrc.2016.05.071

Butler, A., Hoffman, P., Smibert, P., Papalexi, E., \& Satija, R. (2018). Integrating single-cell transcriptomic data across different conditions, technologies, and species. Nature Biotechnology, 36(5), 411-420. doi: 10.1038/nbt.4096

Chalasani, N., Baluyut, A., Ismail, A., Zaman, A., Sood, G., Ghalib, R., ... Hoen, H. (2000). Cholangiocarcinoma in patients with primary sclerosing cholangitis : A multicenter casecontrol study. Hepatology (Baltimore, Md.), 31(1), 7-11. doi: 10.1002/hep.510310103

Chen, H., Gan, Q., Yang, C., Peng, X., Qin, J., Qiu, S., ... Peng, Y. (2019). A novel role of glutathione Stransferase $\mathrm{A} 3$ in inhibiting hepatic stellate cell activation and rat hepatic fibrosis. Journal of Translational Medicine, 17(1), 280. doi: 10.1186/s12967-019-2027-8 
Chen, J., Bardes, E. E., Aronow, B. J., \& Jegga, A. G. (2009). ToppGene Suite for gene list enrichment analysis and candidate gene prioritization. Nucleic Acids Research, 37(Web Server issue), W305-311. doi: 10.1093/nar/gkp427

Cline, M. S., Smoot, M., Cerami, E., Kuchinsky, A., Landys, N., Workman, C., ... Bader, G. D. (2007). Integration of biological networks and gene expression data using Cytoscape. Nature Protocols, 2(10), 2366-2382. doi: 10.1038/nprot.2007.324

Crawford, D. R., Ilic, Z., Guest, I., Milne, G. L., Hayes, J. D., \& Sell, S. (2017). Characterization of liver injury, oval cell proliferation and cholangiocarcinogenesis in glutathione S-transferase A3 knockout mice. Carcinogenesis, 38(7), 717-727. doi: 10.1093/carcin/bgx048

Desterke, C., Lorenzo, H. K., \& Candelier, J.-J. (2021). Text Mining Gene Selection to Understand Pathological Phenotype Using Biological Big Data. In N. Helder I. (Éd.), Bioinformatics. Brisbane (AU): Exon Publications. Consulté à l'adresse http://www.ncbi.nlm.nih.gov/books/NBK569563/

Dwyer, B. J., Jarman, E. J., Gogoi-Tiwari, J., Ferreira-Gonzalez, S., Boulter, L., Guest, R. V., ... Forbes, S. J. (2021). TWEAK/Fn14 signalling promotes cholangiocarcinoma niche formation and progression. Journal of Hepatology, 74(4), 860-872. doi: 10.1016/j.jhep.2020.11.018

Fickert, P., Fuchsbichler, A., Wagner, M., Zollner, G., Kaser, A., Tilg, H., ... Trauner, M. (2004). Regurgitation of bile acids from leaky bile ducts causes sclerosing cholangitis in Mdr2 (Abcb4) knockout mice. Gastroenterology, 127(1), 261-274. doi: 10.1053/j.gastro.2004.04.009

Fickert, P., Pollheimer, M. J., Beuers, U., Lackner, C., Hirschfield, G., Housset, C., ... International PSC Study Group (IPSCSG). (2014). Characterization of animal models for primary sclerosing cholangitis (PSC). Journal of Hepatology, 60(6), 1290-1303. doi: 10.1016/j.jhep.2014.02.006 Flamant, L., Roegiers, E., Pierre, M., Hayez, A., Sterpin, C., De Backer, O., ... Michiels, C. (2012). TMEM45A is essential for hypoxia-induced chemoresistance in breast and liver cancer cells. BMC Cancer, 12, 391. doi: 10.1186/1471-2407-12-391 
Fleuren, W. W. M., \& Alkema, W. (2015). Application of text mining in the biomedical domain. Methods (San Diego, Calif.), 74, 97-106. doi: 10.1016/j.ymeth.2015.01.015

Floreani, A., \& De Martin, S. (2021). Treatment of primary sclerosing cholangitis. Digestive and Liver Disease: Official Journal of the Italian Society of Gastroenterology and the Italian Association for the Study of the Liver, S1590-8658(21)00213-9. doi: 10.1016/j.dld.2021.04.028

Fontaine, J.-F., Priller, F., Barbosa-Silva, A., \& Andrade-Navarro, M. A. (2011). Génie : Literature-based gene prioritization at multi genomic scale. Nucleic Acids Research, 39(Web Server issue), W455-461. doi: 10.1093/nar/gkr246

The Gene Ontology Consortium. (2017). Expansion of the Gene Ontology knowledgebase and resources. Nucleic Acids Research, 45(D1), D331-D338. doi: 10.1093/nar/gkw1108 Goodwin, B., Jones, S. A., Price, R. R., Watson, M. A., McKee, D. D., Moore, L. B., ... Kliewer, S. A. (2000). A regulatory cascade of the nuclear receptors FXR, SHP-1, and LRH-1 represses bile acid biosynthesis. Molecular Cell, 6(3), 517-526. doi: 10.1016/s1097-2765(00)00051-4

Gronemeyer, H., Gustafsson, J.-A., \& Laudet, V. (2004). Principles for modulation of the nuclear receptor superfamily. Nature Reviews. Drug Discovery, 3(11), 950-964. doi: 10.1038/nrd1551

Guo, J., Chen, L., Luo, N., Yang, W., Qu, X., \& Cheng, Z. (2015). Inhibition of TMEM45A suppresses proliferation, induces cell cycle arrest and reduces cell invasion in human ovarian cancer cells. Oncology Reports, 33(6), 3124-3130. doi: 10.3892/or.2015.3902

Hang, S., Paik, D., Yao, L., Kim, E., Trinath, J., Lu, J., ... Huh, J. R. (2019). Bile acid metabolites control TH17 and Treg cell differentiation. Nature, 576(7785), 143-148. doi: 10.1038/s41586-019$1785-z$

Harder, J., Müller, M. J., Fuchs, M., Gumpp, V., Schmitt-Graeff, A., Fischer, R., ... Hasskarl, J. (2013). Inhibitor of differentiation proteins do not influence prognosis of biliary tract cancer. World Journal of Gastroenterology, 19(48), 9334-9342. doi: 10.3748/wjg.v19.i48.9334

Horvath, S., Erhart, W., Brosch, M., Ammerpohl, O., von Schönfels, W., Ahrens, M., ... Hampe, J. (2014). Obesity accelerates epigenetic aging of human liver. Proceedings of the National 
Academy of Sciences of the United States of America, 111(43), 15538-15543. doi:

10.1073/pnas.1412759111

Inagaki, T., Choi, M., Moschetta, A., Peng, L., Cummins, C. L., McDonald, J. G., ... Kliewer, S. A. (2005). Fibroblast growth factor 15 functions as an enterohepatic signal to regulate bile acid homeostasis. Cell Metabolism, 2(4), 217-225. doi: 10.1016/j.cmet.2005.09.001

Johansson, A. S., \& Mannervik, B. (2001). Human glutathione transferase A3-3, a highly efficient catalyst of double-bond isomerization in the biosynthetic pathway of steroid hormones. The Journal of Biological Chemistry, 276(35), 33061-33065. doi: 10.1074/jbc.M104539200

Karlsen, T. H., Folseraas, T., Thorburn, D., \& Vesterhus, M. (2017). Primary sclerosing cholangitis-A comprehensive review. Journal of Hepatology, 67(6), 1298-1323. doi:

10.1016/j.jhep.2017.07.022

Krallinger, M., Valencia, A., \& Hirschman, L. (2008). Linking genes to literature : Text mining, information extraction, and retrieval applications for biology. Genome Biology, 9 Suppl 2, S8. doi: $10.1186 / g b-2008-9-s 2-s 8$

Lazaridis, K. N., \& LaRusso, N. F. (2016). Primary Sclerosing Cholangitis. The New England Journal of Medicine, 375(12), 1161-1170. doi: 10.1056/NEJMra1506330

Lê, S., Josse, J., \& Husson, F. (2008). FactoMineR: An R Package for Multivariate Analysis. Journal of Statistical Software, 25(1), 1-18.

Lee, S., Stewart, S., Nagtegaal, I., Luo, J., Wu, Y., Colditz, G., ... Allred, D. C. (2012). Differentially expressed genes regulating the progression of ductal carcinoma in situ to invasive breast cancer. Cancer Research, 72(17), 4574-4586. doi: 10.1158/0008-5472.CAN-12-0636

Manawapat-Klopfer, A., Thomsen, L. T., Martus, P., Munk, C., Russ, R., Gmuender, H., ... Iftner, T. (2016). TMEM45A, SERPINB5 and p16INK4A transcript levels are predictive for development of high-grade cervical lesions. American Journal of Cancer Research, 6(7), 1524-1536. 
Mertz, A., Nguyen, N. A., Katsanos, K. H., \& Kwok, R. M. (2019). Primary sclerosing cholangitis and inflammatory bowel disease comorbidity : An update of the evidence. Annals of Gastroenterology, 32(2), 124-133. doi: 10.20524/aog.2019.0344

Molodecky, N. A., Kareemi, H., Parab, R., Barkema, H. W., Quan, H., Myers, R. P., \& Kaplan, G. G. (2011). Incidence of primary sclerosing cholangitis : A systematic review and meta-analysis. Hepatology (Baltimore, Md.), 53(5), 1590-1599. doi: 10.1002/hep.24247

Ozdil, B., Cosar, A., Akkiz, H., Sandikci, M., \& Kece, C. (2011). New therapeutic option with Nacetylcysteine for primary sclerosing cholangitis : Two case reports. American Journal of Therapeutics, 18(3), e71-74. doi: 10.1097/MJT.0b013e3181c42758

Pavlidis, P., \& Noble, W. S. (2001). Analysis of strain and regional variation in gene expression in mouse brain. Genome Biology, 2(10), RESEARCH0042.

Pirola, C. J., \& Sookoian, S. (2021). The lipidome in nonalcoholic fatty liver disease : Actionable targets. Journal of Lipid Research, 62, 100073. doi: 10.1016/j.jlr.2021.100073

Reich, M., Spomer, L., Klindt, C., Fuchs, K., Stindt, J., Deutschmann, K., ... Keitel, V. (2021). Downregulation of TGR5 (GPBAR1) in biliary epithelial cells contributes to the pathogenesis of sclerosing cholangitis. Journal of Hepatology, S0168-8278(21)00244-0. doi: 10.1016/j.jhep.2021.03.029

Ritchie, M. E., Phipson, B., Wu, D., Hu, Y., Law, C. W., Shi, W., \& Smyth, G. K. (2015). Limma powers differential expression analyses for RNA-sequencing and microarray studies. Nucleic Acids Research, 43(7), e47. doi: 10.1093/nar/gkv007

Rizvi, S., Eaton, J. E., \& Gores, G. J. (2015). Primary Sclerosing Cholangitis as a Premalignant Biliary Tract Disease : Surveillance and Management. Clinical Gastroenterology and Hepatology: The Official Clinical Practice Journal of the American Gastroenterological Association, 13(12), 2152-2165. doi: 10.1016/j.cgh.2015.05.035

Rühlemann, M., Liwinski, T., Heinsen, F.-A., Bang, C., Zenouzi, R., Kummen, M., ... Franke, A. (2019). Consistent alterations in faecal microbiomes of patients with primary sclerosing cholangitis 
independent of associated colitis. Alimentary Pharmacology \& Therapeutics, 50(5), 580-589. doi: 10.1111/apt.15375

Sabino, J., Vieira-Silva, S., Machiels, K., Joossens, M., Falony, G., Ballet, V., ... Raes, J. (2016). Primary sclerosing cholangitis is characterised by intestinal dysbiosis independent from IBD. Gut, 65(10), 1681-1689. doi: 10.1136/gutjnl-2015-311004

Sakaguchi, S., Miyara, M., Costantino, C. M., \& Hafler, D. A. (2010). FOXP3+ regulatory T cells in the human immune system. Nature Reviews. Immunology, 10(7), 490-500. doi: 10.1038/nri2785 Sayers, E. (2010). Entrez Programming Utilities Help (https://www.ncbi.nlm.nih.gov/books/NBK25501/). National Center for Biotechnology Information (US). (https://www.ncbi.nlm.nih.gov/books/NBK25501/). Consulté à l'adresse https://www.ncbi.nlm.nih.gov/books/NBK25501/

Sayers, E. W., Barrett, T., Benson, D. A., Bryant, S. H., Canese, K., Chetvernin, V., ... Ye, J. (2009). Database resources of the National Center for Biotechnology Information. Nucleic Acids Research, 37(Database issue), D5-15. doi: 10.1093/nar/gkn741

Sebode, M., Peiseler, M., Franke, B., Schwinge, D., Schoknecht, T., Wortmann, F., ... Schramm, C. (2014). Reduced FOXP3(+) regulatory T cells in patients with primary sclerosing cholangitis are associated with IL2RA gene polymorphisms. Journal of Hepatology, 60(5), 1010-1016. doi: 10.1016/j.jhep.2013.12.027

Seol, W., Choi, H. S., \& Moore, D. D. (1996). An orphan nuclear hormone receptor that lacks a DNA binding domain and heterodimerizes with other receptors. Science (New York, N.Y.), 272(5266), 1336-1339. doi: 10.1126/science.272.5266.1336

Sinal, C. J., Tohkin, M., Miyata, M., Ward, J. M., Lambert, G., \& Gonzalez, F. J. (2000). Targeted disruption of the nuclear receptor FXR/BAR impairs bile acid and lipid homeostasis. Cell, 102(6), 731-744. doi: 10.1016/s0092-8674(00)00062-3

Subramanian, A., Tamayo, P., Mootha, V. K., Mukherjee, S., Ebert, B. L., Gillette, M. A., ... Mesirov, J. P. (2005). Gene set enrichment analysis : A knowledge-based approach for interpreting 
genome-wide expression profiles. Proceedings of the National Academy of Sciences of the United States of America, 102(43), 15545-15550. doi: 10.1073/pnas.0506580102

Sun, W., Qiu, G., Zou, Y., Cai, Z., Wang, P., Lin, X., ... Hu, G. (2015). Knockdown of TMEM45A inhibits the proliferation, migration and invasion of glioma cells. International Journal of Clinical and Experimental Pathology, 8(10), 12657-12667.

Szklarczyk, D., Morris, J. H., Cook, H., Kuhn, M., Wyder, S., Simonovic, M., ... von Mering, C. (2017). The STRING database in 2017 : Quality-controlled protein-protein association networks, made broadly accessible. Nucleic Acids Research, 45(D1), D362-D368. doi: $10.1093 /$ nar/gkw937

Tahboub Amawi, A. D., Tremaine, W. J., \& Venkatesh, S. K. (2020). Pembrolizumab-Induced Sclerosing Cholangitis. Clinical Gastroenterology and Hepatology: The Official Clinical Practice Journal of the American Gastroenterological Association, S1542-3565(20)31638-4. doi: 10.1016/j.cgh.2020.11.048

Tietz-Bogert, P. S., Kim, M., Cheung, A., Tabibian, J. H., Heimbach, J. K., Rosen, C. B., ... O’Hara, S. P. (2018). Metabolomic Profiling of Portal Blood and Bile Reveals Metabolic Signatures of Primary Sclerosing Cholangitis. International Journal of Molecular Sciences, 19(10), E3188. doi: $10.3390 /$ ijms19103188

Trapnell, C., Cacchiarelli, D., Grimsby, J., Pokharel, P., Li, S., Morse, M., ... Rinn, J. L. (2014). The dynamics and regulators of cell fate decisions are revealed by pseudotemporal ordering of single cells. Nature Biotechnology, 32(4), 381-386. doi: 10.1038/nbt.2859

Vestentoft, P. S., Jelnes, P., Hopkinson, B. M., Vainer, B., Møllgård, K., Quistorff, B., \& Bisgaard, H. C. (2011). Three-dimensional reconstructions of intrahepatic bile duct tubulogenesis in human liver. BMC Developmental Biology, 11, 56. doi: 10.1186/1471-213X-11-56

Wickham, H. (2009). ggplot2 : Elegant Graphics for Data Analysis. Springer-Verlag New York. 
Wrzesiński, T., Szelag, M., Cieślikowski, W. A., Ida, A., Giles, R., Zodro, E., ... Wesoly, J. (2015).

Expression of pre-selected TMEMs with predicted ER localization as potential classifiers of ccRCC tumors. BMC Cancer, 15, 518. doi: 10.1186/s12885-015-1530-4

Xia, J., Gill, E. E., \& Hancock, R. E. W. (2015). NetworkAnalyst for statistical, visual and network-based meta-analysis of gene expression data. Nature Protocols, 10(6), 823-844. doi: $10.1038 /$ nprot.2015.052

Xu, C., \& Su, Z. (2015). Identification of cell types from single-cell transcriptomes using a novel clustering method. Bioinformatics (Oxford, England), 31(12), 1974-1980. doi: 10.1093/bioinformatics/btv088

Yang, Y., Sharma, R., Zimniak, P., \& Awasthi, Y. C. (2002). Role of alpha class glutathione Stransferases as antioxidant enzymes in rodent tissues. Toxicology and Applied Pharmacology, 182(2), 105-115. doi: 10.1006/taap.2002.9450

Zhan, L., Liu, H.-X., Fang, Y., Kong, B., He, Y., Zhong, X.-B., ... Guo, G. L. (2014). Genome-wide binding and transcriptome analysis of human farnesoid $\mathrm{X}$ receptor in primary human hepatocytes. PloS One, 9(9), e105930. doi: 10.1371/journal.pone.0105930

Zhang, Y., Hagedorn, C. H., \& Wang, L. (2011). Role of nuclear receptor SHP in metabolism and cancer. Biochimica Et Biophysica Acta, 1812(8), 893-908. doi: 10.1016/j.bbadis.2010.10.006 


\section{FIGURE LEGENDS}

Figure 1: workflow of bioinformatics analyses: this figure describes the workflow of the bioinformatics process performed on data used in this work.

Figure 2: Abnormal hepatobiliary system physiology in liver biopsies of Primary sclerosing cholangitis liver biopsy: from A to $\mathrm{E}$ : investigations on transcriptome dataset GSE61256; A/ principal component analysis performed with 507 detected symptom related genes on liver biopsy transcriptome from distinct group of diseases and controls, PSC: primary sclerosing cholangitis, PBC: primary biliary cholangitis, NAFLD: Nonalcoholic fatty liver disease, NASH: Nonalcoholic steatohepatitis; B/ Volcanoplot of differential expressed genes (DEGs) between PSC liver and other liver biopsies, C/ Expression heatmap of significant up regulated genes in PSC liver samples; D/ Functional enrichment performed on Mouse Phenotype database with genes up regulated in PSC liver samples; E/ Functional enrichment network with PSC up regulated genes and implicated in hepatobiliary mouse phenotype.

Figure 3: Hepatic regulation of immune response and intracellular receptor signaling under FXR dependency: from A to D: investigations on transcriptome dataset GSE54557; A/ qqplot of p-values obtained by Pavlidis Template Matching (PTM) algorithm with symptom related genes for liver FXR regulation dependency; B/ Principal component analysis performed with PSC symptom related genes under liver FXR regulation dependency, KO: knock-out, GW4064: FXR agonist; C/ Expression heatmap for PSC symptom related genes found positively correlated to hepatic FXR regulation; D/ Protein-protein interaction network based on PSC symptom related genes up regulated in PSC liver samples with colored function inference of Gene Ontology Biological Process (GO-BP) database: in green enrichment of immune response function and in blue intracellular receptor signaling function.

Figure 4: Nrb02 is the best predictive marker regulated in liver of primary sclerosing cholangitis under FXR dependency: A/Venn diagram merging FXR liver regulation dependency of PSC related genes found up regulated in PSC human liver biopsies; B/ Supervised machine learning misclassification plot by class obtained with PSC symptom related genes to stratified PSC liver samples from other control samples in GSE61256; C/ Ranked predictive scores obtained by machine learning on GSE61256 dataset with PSC symptom related genes, FXR hepatic regulation dependency is highlighted in pink for concerned genes; $\mathrm{D} / \mathrm{Boxplot}$ of $\mathrm{Nr} 0 \mathrm{~b} 2$ expression regulation through liver group samples and stratified on gender (GSE61256); E/ Multivariate model based on NrOb2 expression: exponential coefficients and their confident intervals (95 percent) were drawn for each covariates.

Figure 5: Up regulation of $\mathrm{Nrb02}$ in cholangiocyte compartment of Abcb4-/- liver: from A to G: investigations on whole liver single cell transcriptome dataset GSE168758; A/ Uniform Manifold Approximation and Projection (UMAP) dimension reduction with identification of the distinct cell compartments in whole liver samples: colors reflect 15 distinct cluster cell communities; B/ UMAP with genotype stratification: WT: wildtype, Abcb4-/-: Abcb4 knock-out; C/ Violinplot of Sox9 expression in whole liver stratified on genotypes; D/ Sox9 feature plot stratified on genotypes: blue for positive expression; E/ Violinplot of $\mathrm{NrOb} 2$ expression in whole liver stratified on genotypes; F/ NrOb2 feature plot 
stratified on genotypes: blue for positive expression; G/ violin of Nr0b2 expression stratified on cluster cell communities and genotypes.

Figure 6: metabolic regulation during cell trajectory of Abcb4-/- cholangiocytes: from A to F: downsampling of Abcb4-/- identified cholangiocytes in liver single cell transcriptome dataset GSE168758; A/ Pie chart of cell stratification depending of alternative expression of NrOb2 and Sox9 in cholangiocyte of Abcb4-/- liver samples; B/ Abcb4-/- cholangiocyte single cell trajectory identified on altenative expression of $\mathrm{NrOb} 2$ and Sox9; C/ Pseudotime expression heatmap of genes found significantly regulated on NrOb2-Sox9 single cell trajectory of Abcb4-/- cholangiocytes: green cluster follow Sox9 and Nr0b2 pseudotime expression; D/ protein-protein interaction network identified on pseudotime green cluster: blue color characterized network inference of positive regulation of metabolism process; E/ Barplot of functional enrichment performed on pseudotime green cluster with Gene Ontology Biological Process database, FDR: False Discovery Rate; F/Functional enrichment network performed with best metabolism function enriched on pseudotime green cluster. 


\section{SUPPLEMENTAL MATERIAL Supplemental Figures}

Supplemental Figure 1: Stratification of PSC and PBC liver transcriptomes by unsupervised analysis: Second principal map of principal component analysis performed on liver transcriptomes of dataset GSE61260 with PCS related genes found by text mining.

Supplemental Figure 2: Single cell heterogeneity of clusters identified in WT and Abcbc4-/- livers: A/ whole liver UMAP dimension reduction with summary of the cell cluster indentifications; B/ Cldn7 featureplot which identified cholangiocyte cell subgroup; C/ Apoc4 featureplot which identified hepatocyte cell subgroup; D/ Kdr featureplot which identified endothelial cell subgroup; E/ Cd68 featureplot which identified macrophage cell subgroup; F/ Cd8a featureplot which identified T-lymphocyte cell subgroup; G/ Ms4a1 Kdr featureplot which identified B-lymphocyte cell subgroup.

Supplemental Figure 3: Single cell differential expression of Sox9, Nr0b2, Epcam in cholangiocyte clusters of WT and Abcb4-/- livers: A/ Violinplot of Sox9 expression in cholangiocyte cluters 0-5-11 stratified on phenotype wildtype versus Abcb4-/-; B/ Violinplot of $\mathrm{Nr} 0 \mathrm{~b} 2$ expression in cholangiocyte cluters $0-5-11$ stratified on phenotype wildtype versus Abcb4-/-; C/ Violinplot of Epcam expression in cholangiocyte cluters 0-5-11 stratified on phenotype wildtype versus Abcb4-/-;

Supplemental Figure 4: Pseudotime transformation of cholangiocyte cell trajectory in Abcb4 -/- livers: A/t-SNE plot of Abcb4-/- cholangiocyte with group stratification based on alternative expression of Sox 9 and $\mathrm{NrOb} 2$; B/ t-SNE plot of Abcb4-/- cholangiocyte with group stratification identified in Seurat clustering: clusters 0-5-11; C/ pseudotime tree with group stratification identified in Seurat clustering: clusters 0-5-11; D/ pseudotime tree with expression of sox 9 as dot size and group stratification as dot color; E/ pseudotime tree with expression of $\mathrm{Nr} 0 \mathrm{~b} 2$ as dot size and group stratification as dot color; F/ pseudotime expression plot for markers found closely regulated with $\mathrm{NrOb} 2$ on pseudotime cell trajectory

\section{Supplemental Tables}

Supplemental Table 1: Textmining table of the common 525 ranked genes found in Pubmed with symptom keywords: this table describes the 525 ranked genes obtained by text mining with 'Génie' algorithm against the three MESH terms: biliary inflammation, biliary fibrosis, biliary stasis, respective ranks, p-values and collected PMID identifiers for each gene and each MESH term are presented

Supplemental Table 2: Symptoms related genes found differentially expressed in liver from primary sclerosing cholangitis patients: Differential expressed gene analysis results (logarithm Fold Change, Average Epression and Adjust p-values) are presented with respective machine learning predictive score for supervized sample categories of GSE61256 dataset: PSC versus other liver samples)

Supplemental Table 3: Pavlidis Template Matching for FXR dependency in liver transcriptome: Pavlidis template analysis results to describe FXR regulation dependency of PSC genes in liver samples of GSE54557 dataset. For each gene R-Pearson correlation coefficient and its respective $\mathrm{p}$-value are presented in this table. 
Supplemental Table 4: Best one hundred genes found to be significant pseudotime trajectory of Abcb4-/- cholangiocytes : Best one hundred ranked genes found as significant on pseudotime cell trajectory of cholangiocytes Abcb4-/- based on alternative expression of Nr0b2 and Sox9 in GSE168758 
525 genes related to PSC symptomatology (textmining)

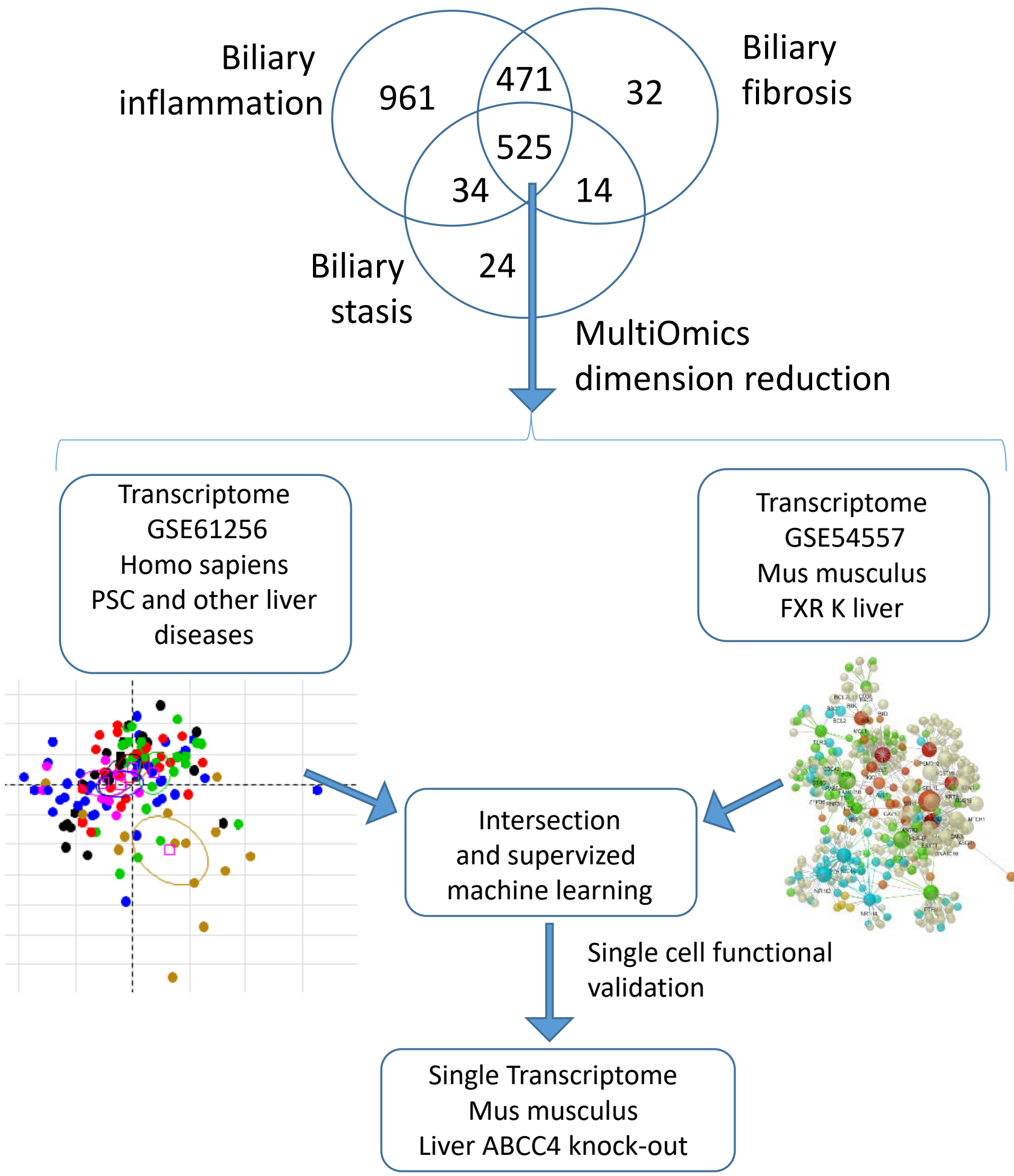

Whole liver heterogeneity

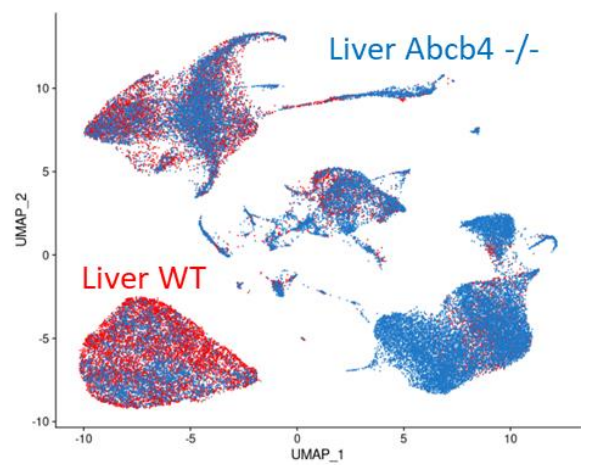

Cholangiocyte trajectory

Figure 1 

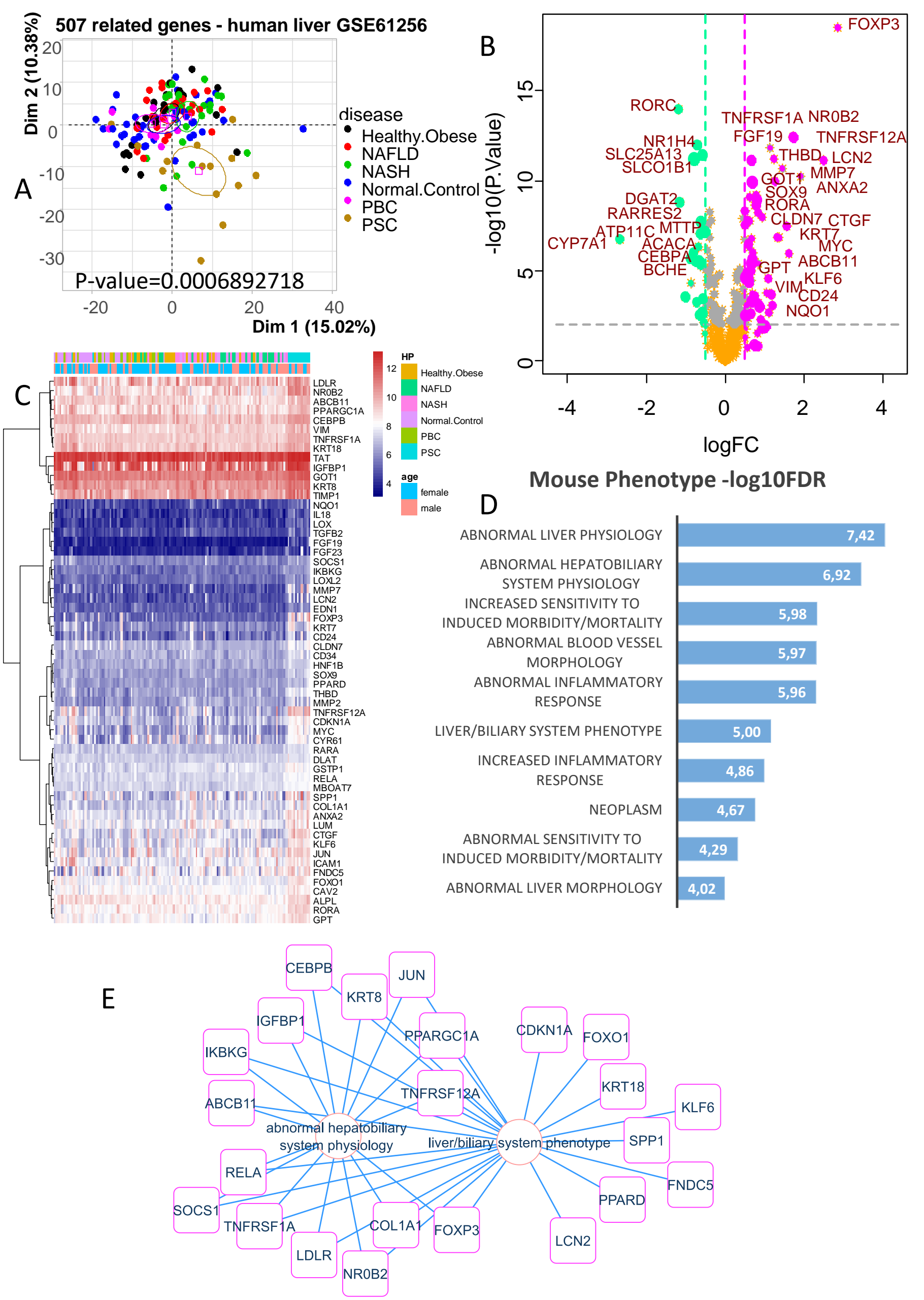
A

PTM in liver FXR KO

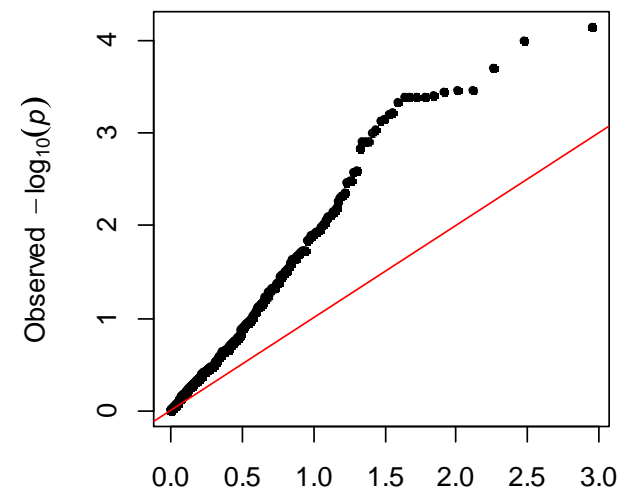

Expected $-\log _{10}(p)$
B

FXR liver dependency for PSC related genes GSE54557

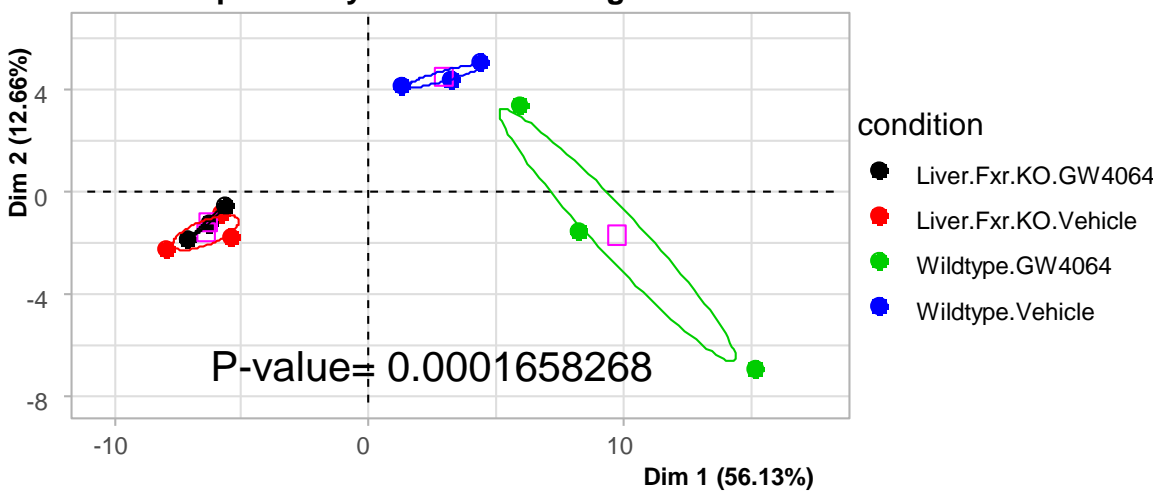

C
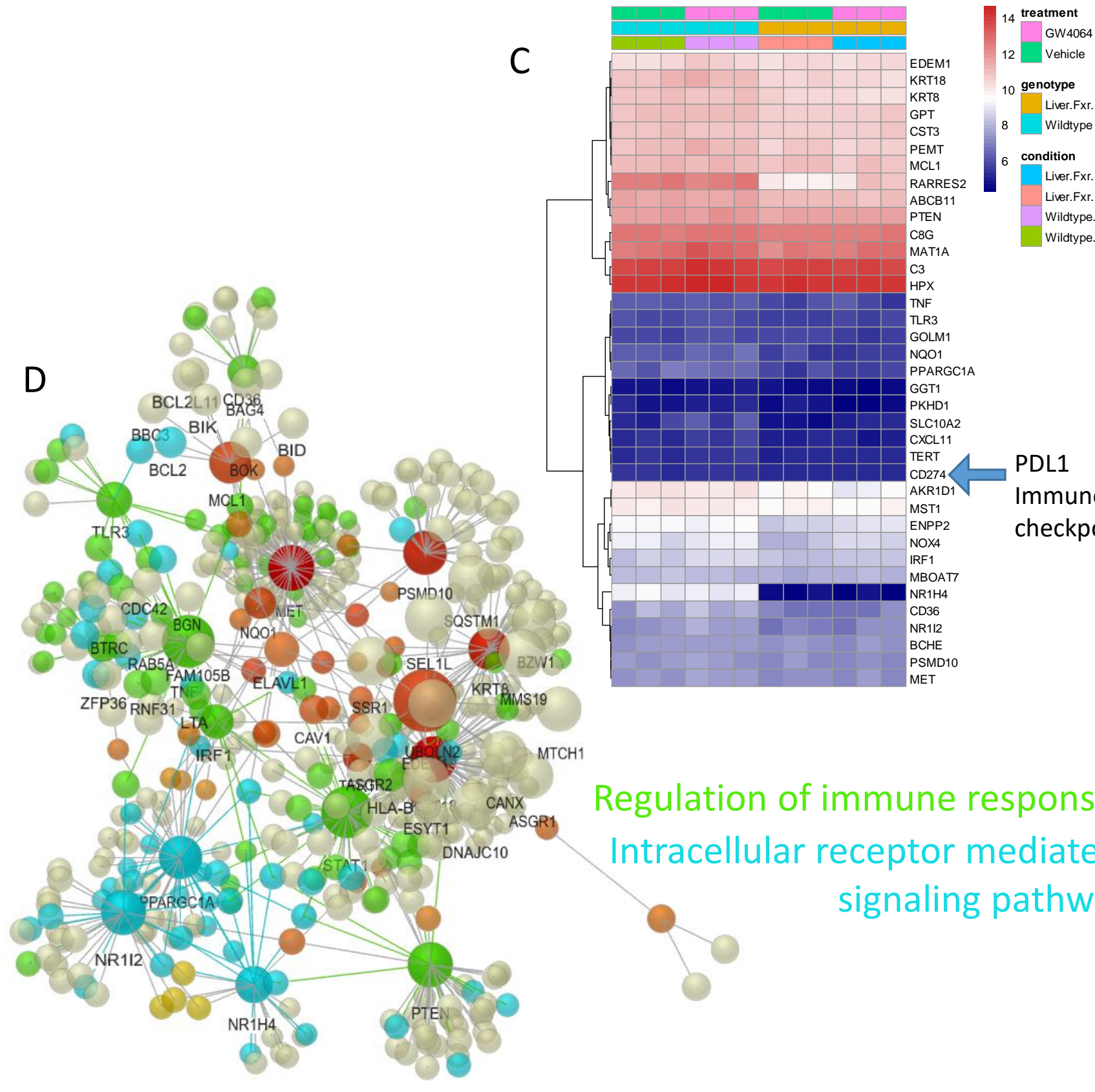

$$
\begin{aligned}
& \begin{array}{l|l}
\text { KRT18 } & 10 \text { genotype } \\
\text { KRT8 } &
\end{array} \\
& \begin{array}{ll}
\text { KRT8 } & 10 \text { Liver.Fxr.KO } \\
\text { GPT } &
\end{array} \\
& 8 \quad \text { Wildtype } \\
& 6 \text { condition }
\end{aligned}
$$

Regulation of immune response Intracellular receptor mediated

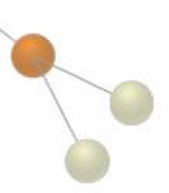

Figure 3 

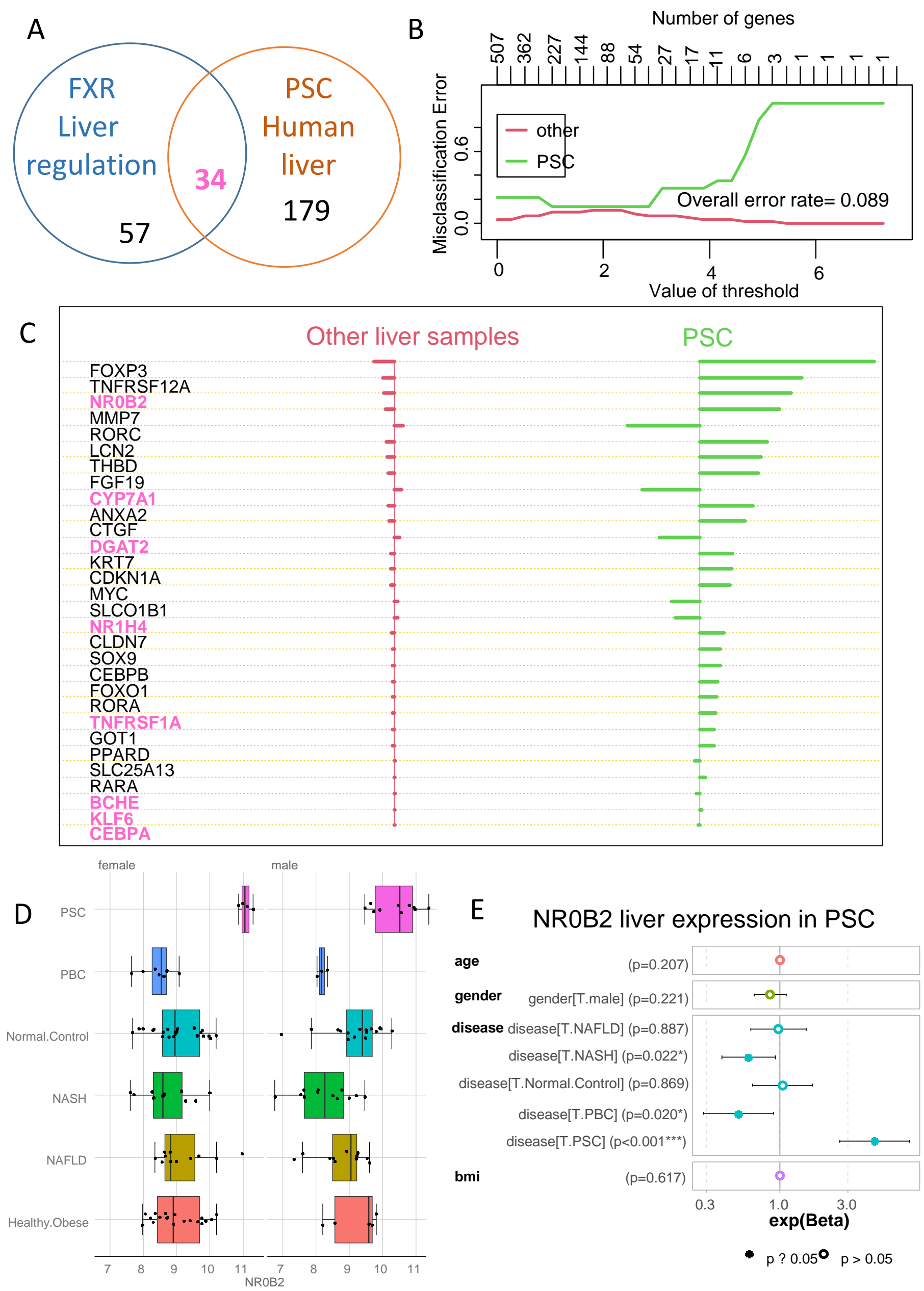

Figure 4 
A
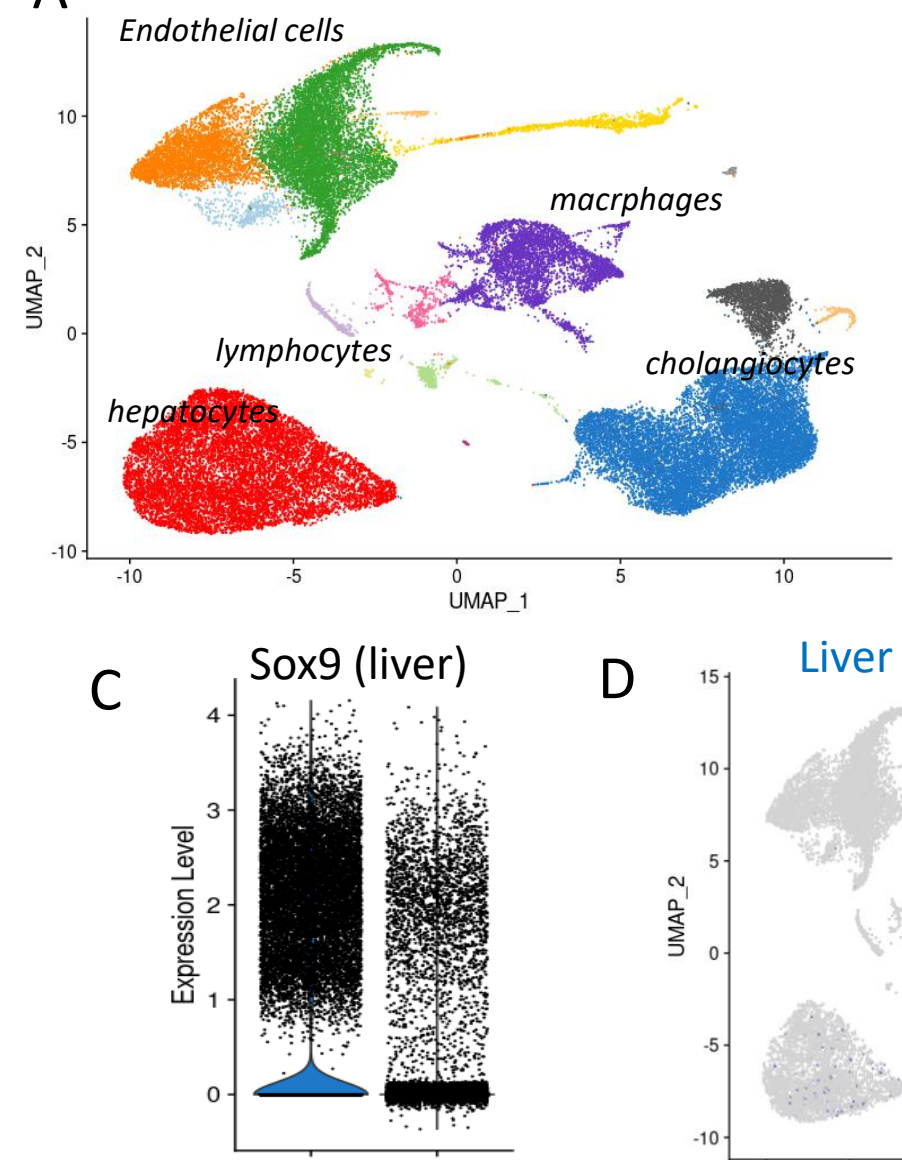

Abcb4-/- WT

E

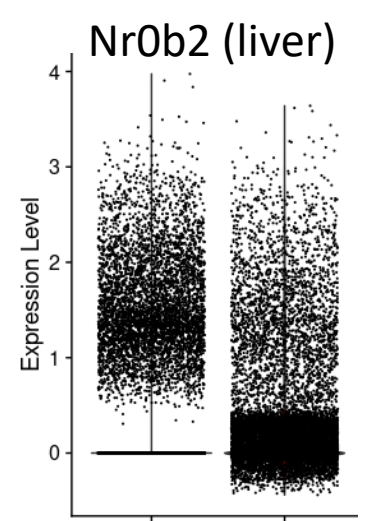

Abcb4-/- WT
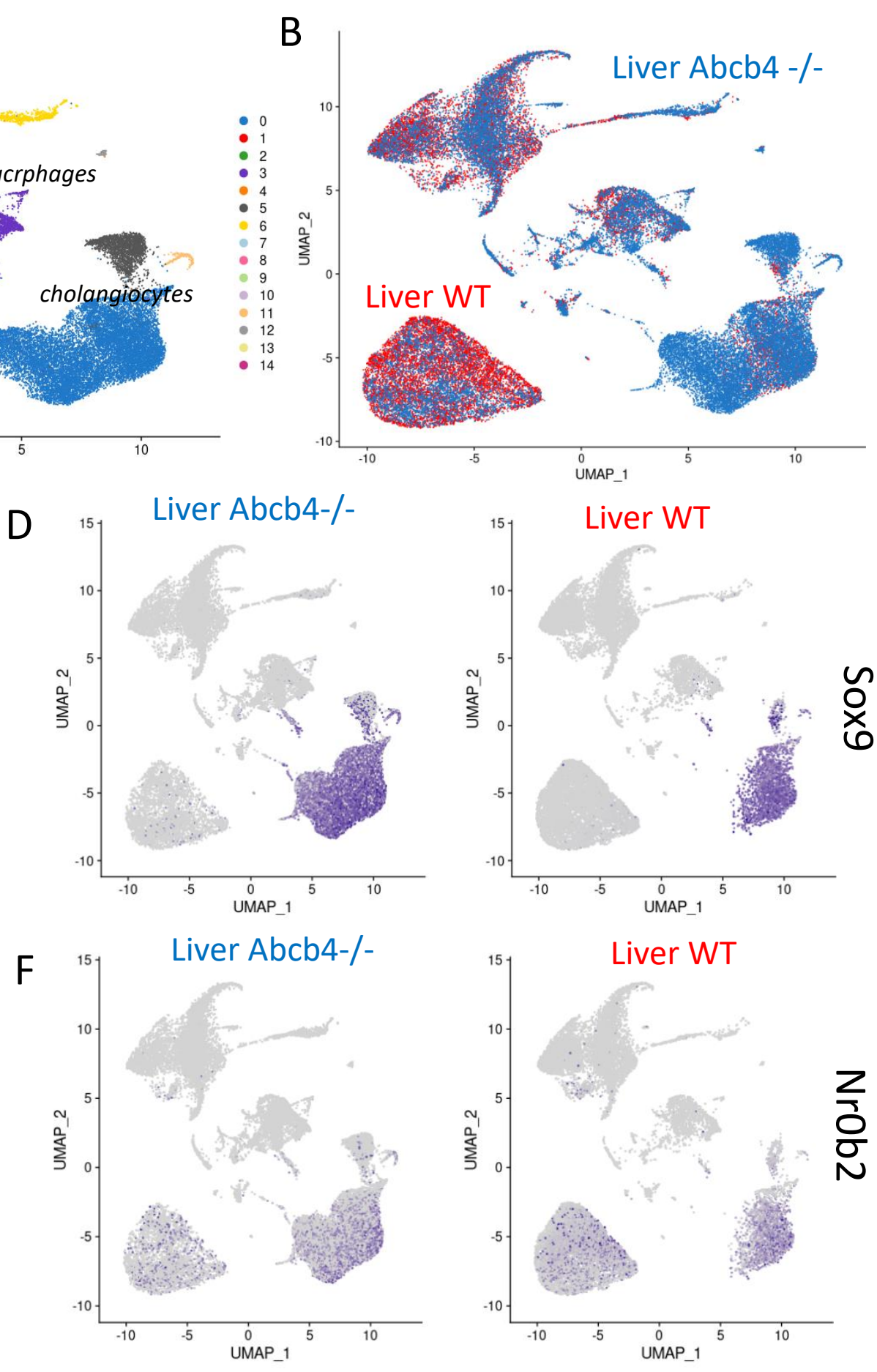

G

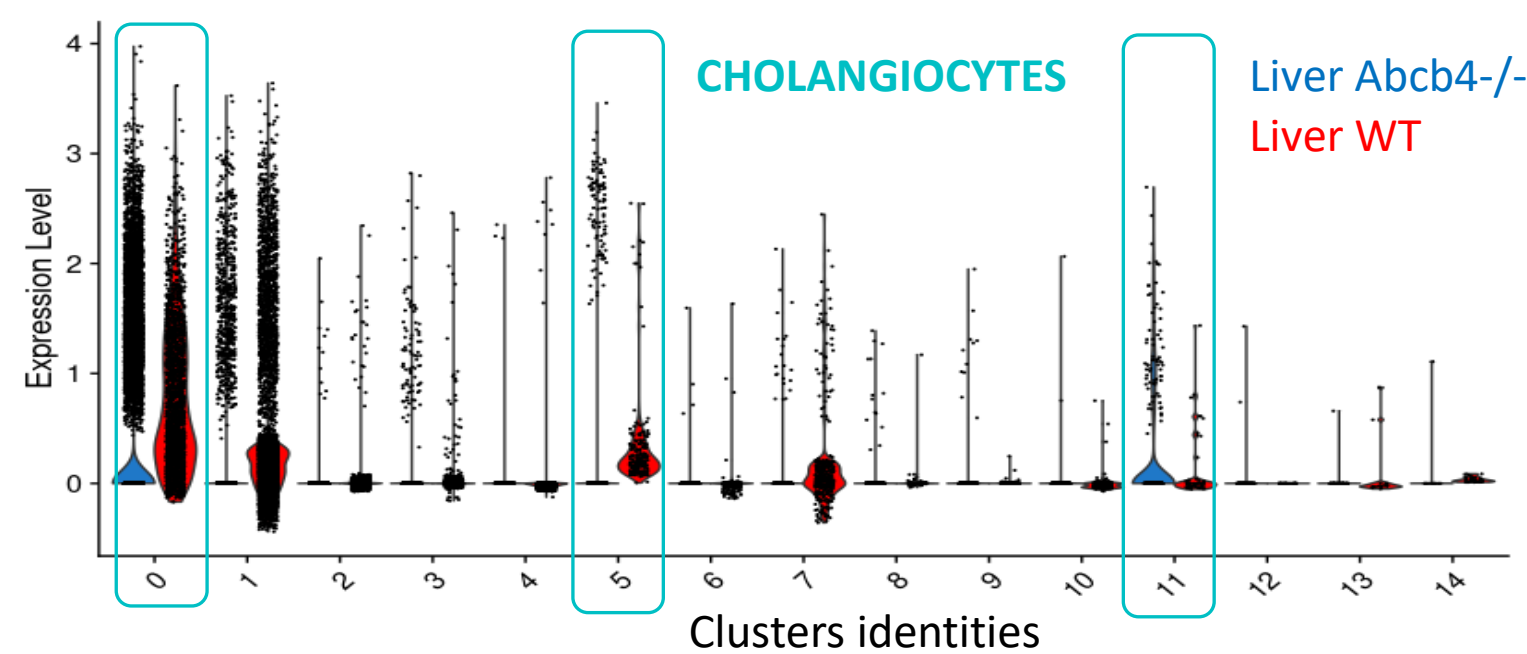

Figure 5 


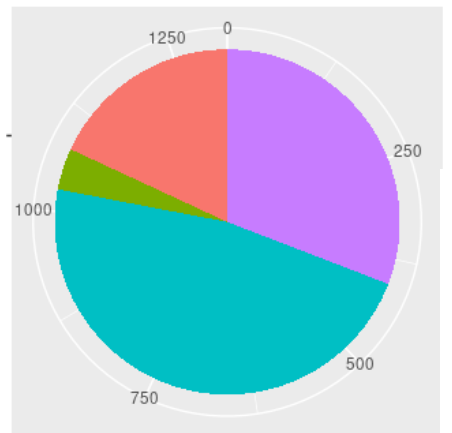

A

C
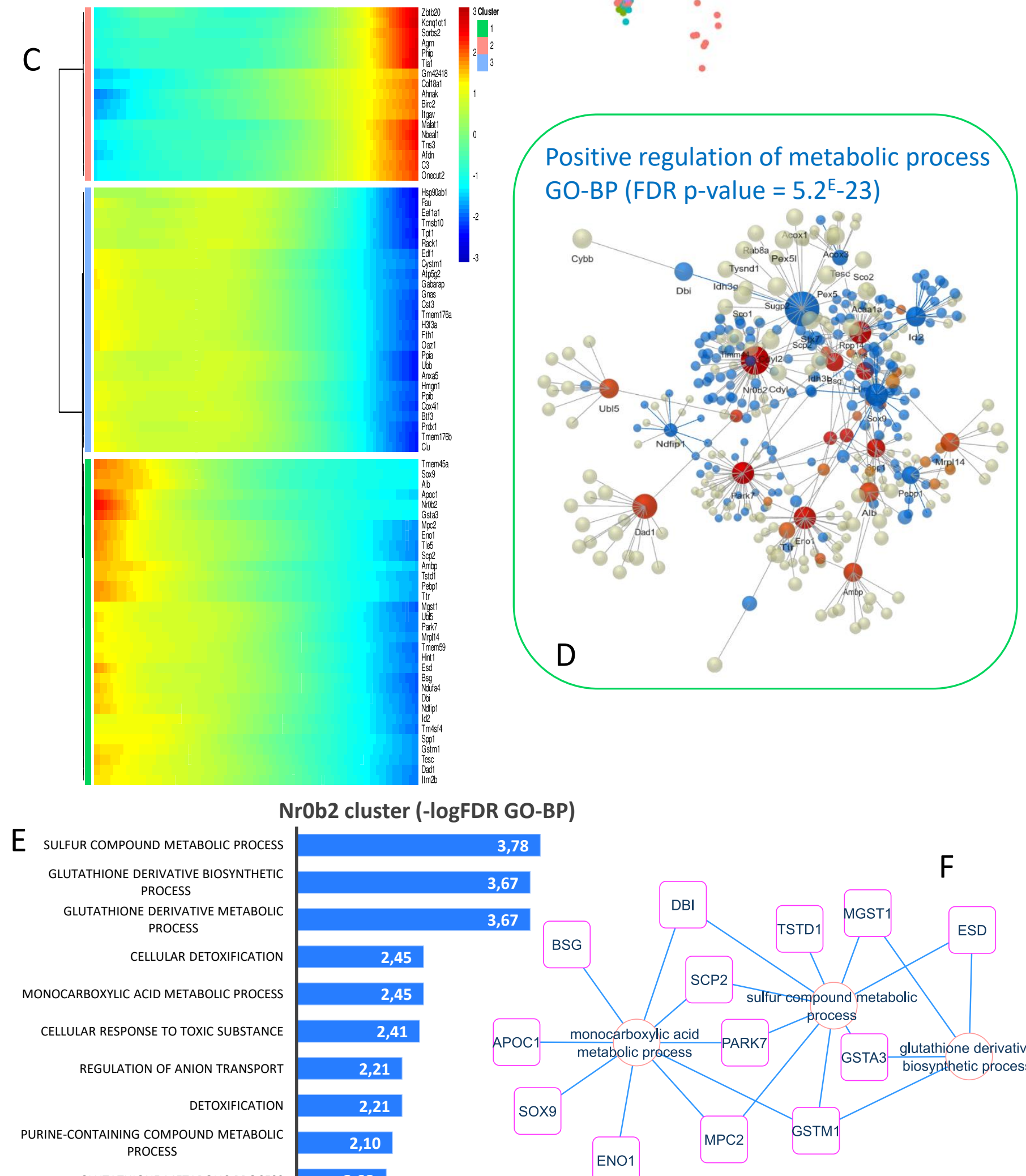

NrOb2 cluster (-logFDR GO-BP)

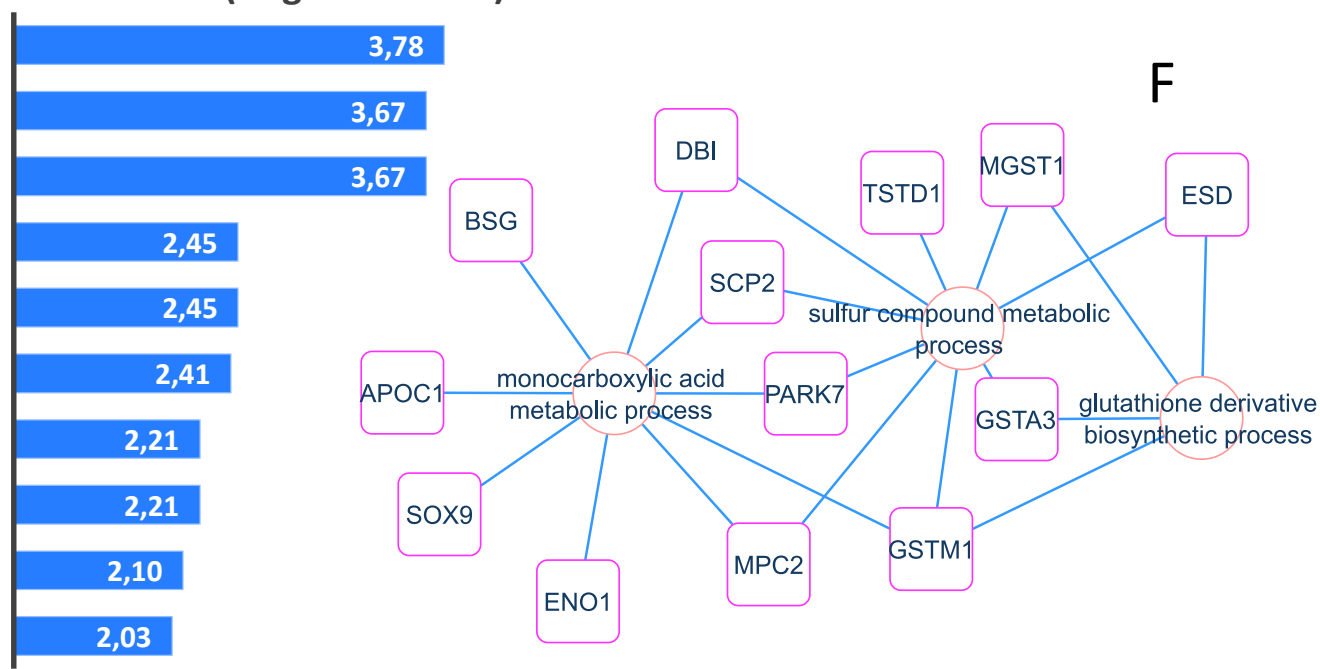

Figure 6

Sox9-NrOb2Sox9-NrOb2+ Sox9+NrOb2Sox9+NrOb2+

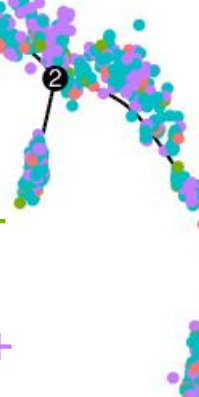

B
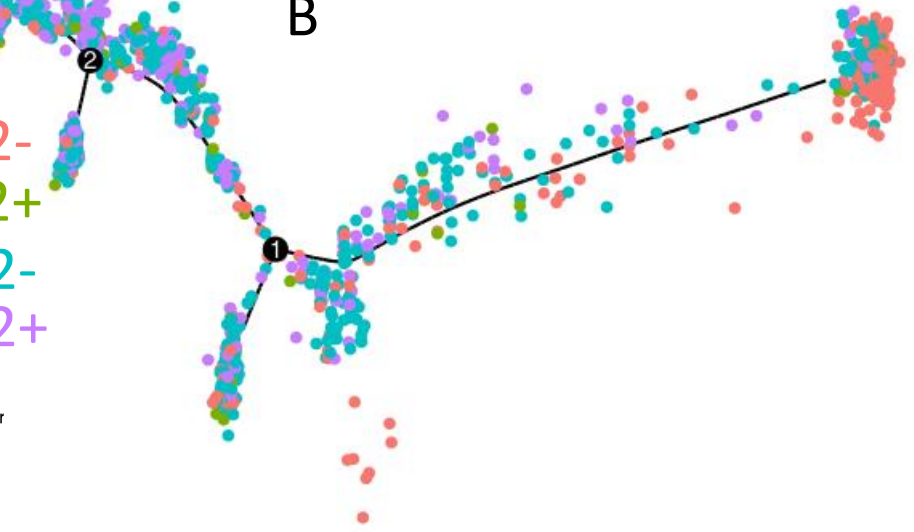

Positive regulation of metabolic process

GO-BP (FDR p-value $=5.2^{\mathrm{E}}-23$ ) 


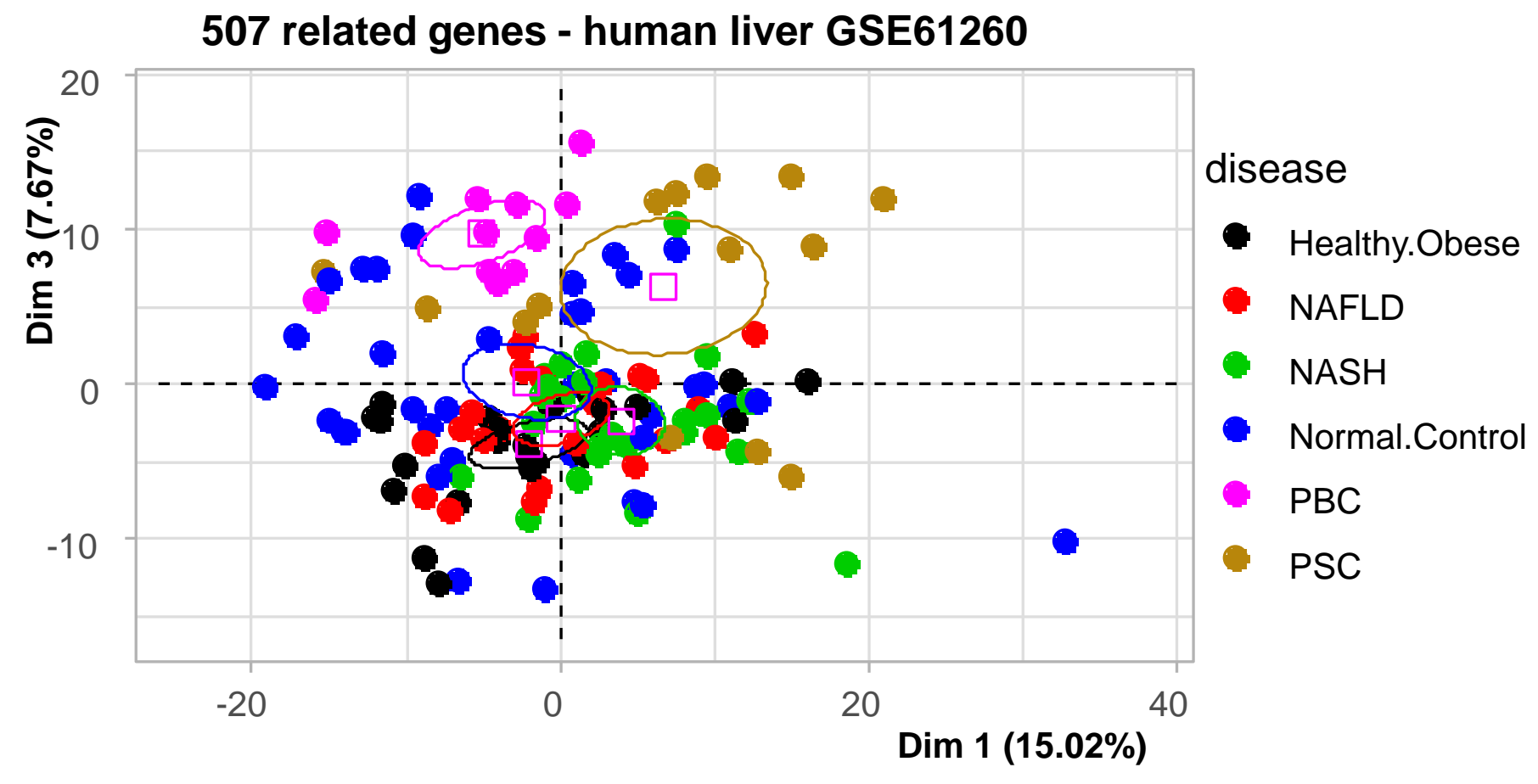

Supplemental Figure 1 


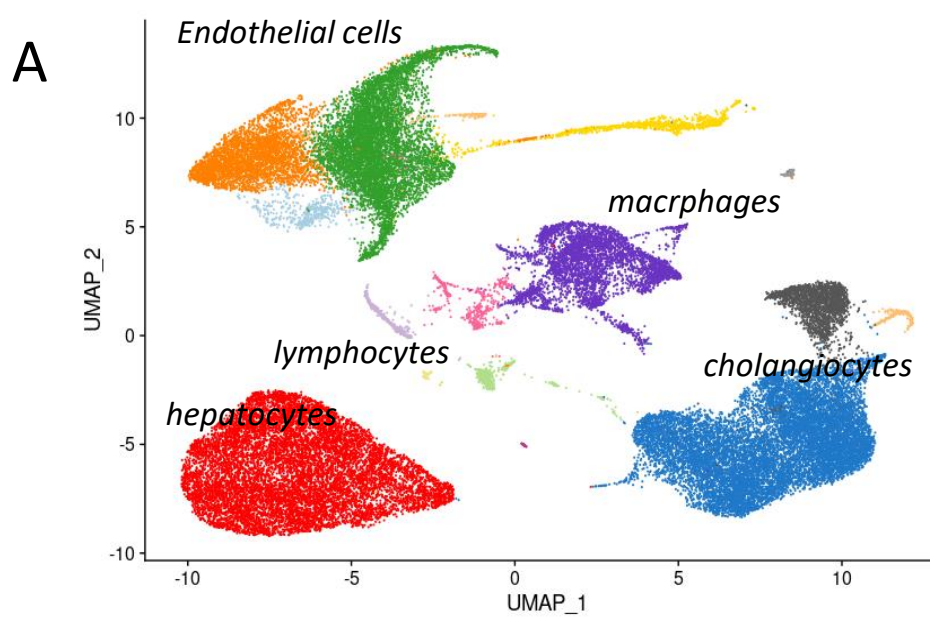

C
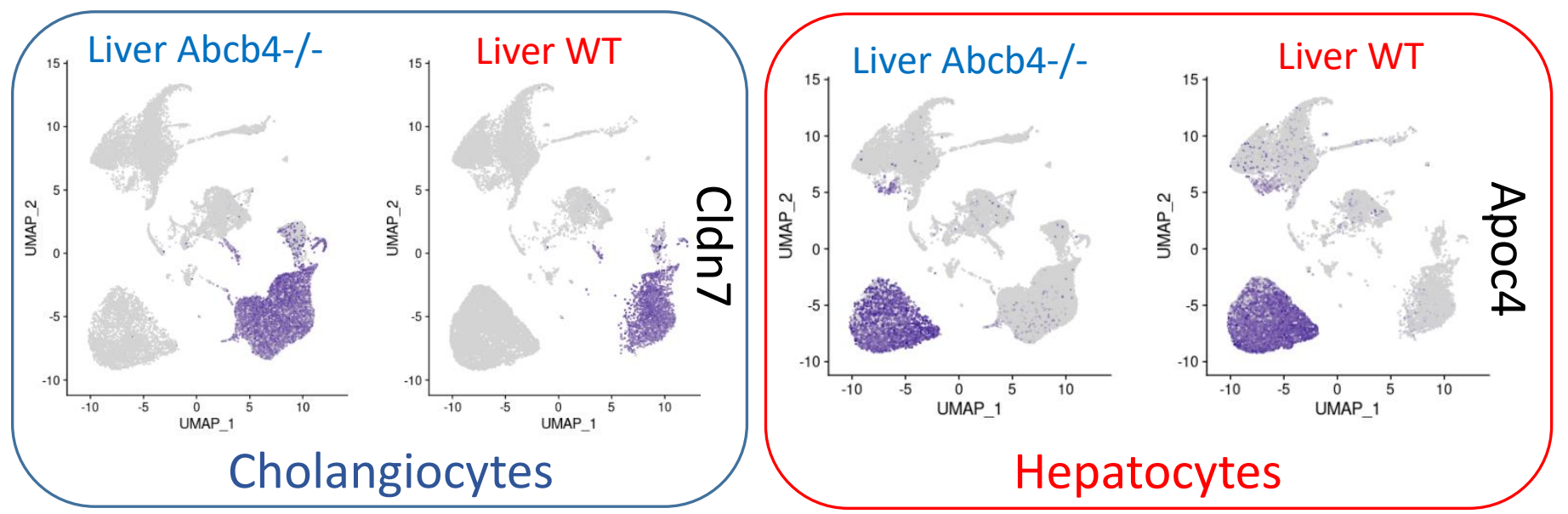

D

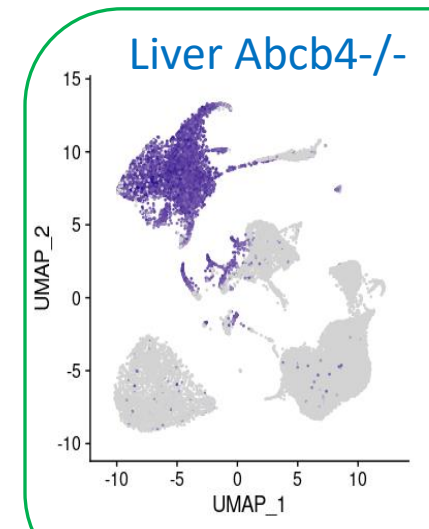

Endothelial cells

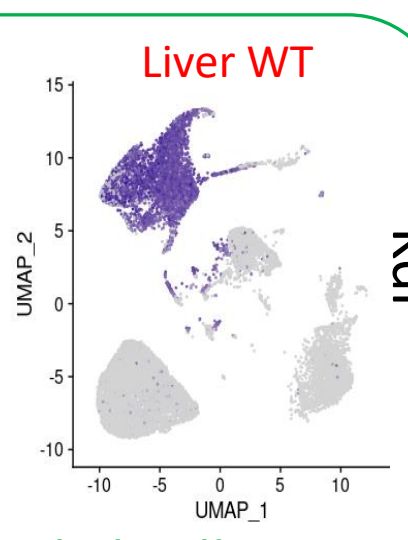

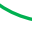

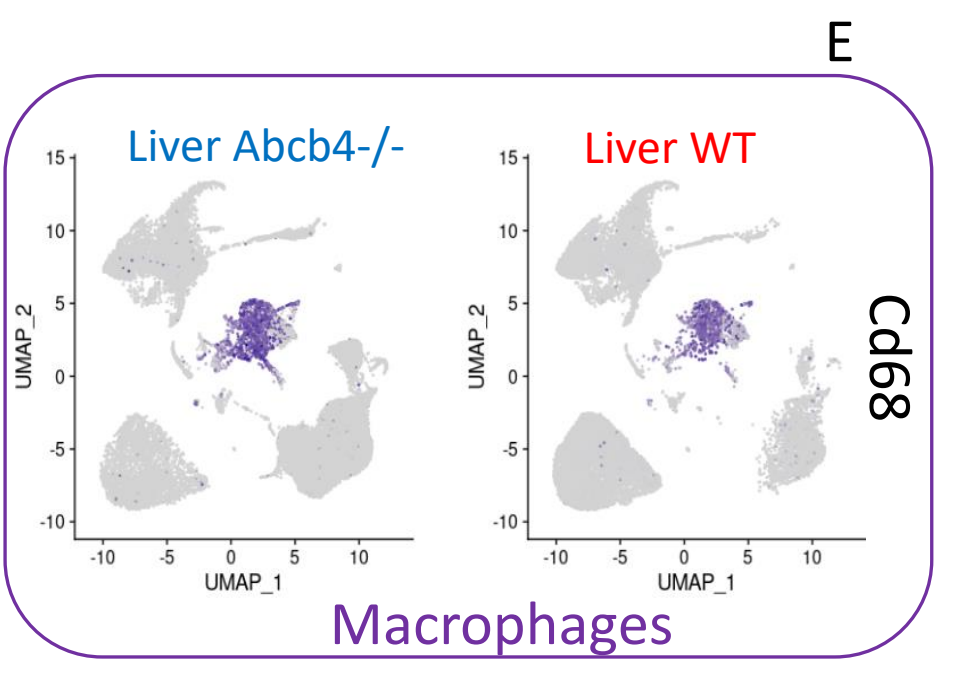

F

G
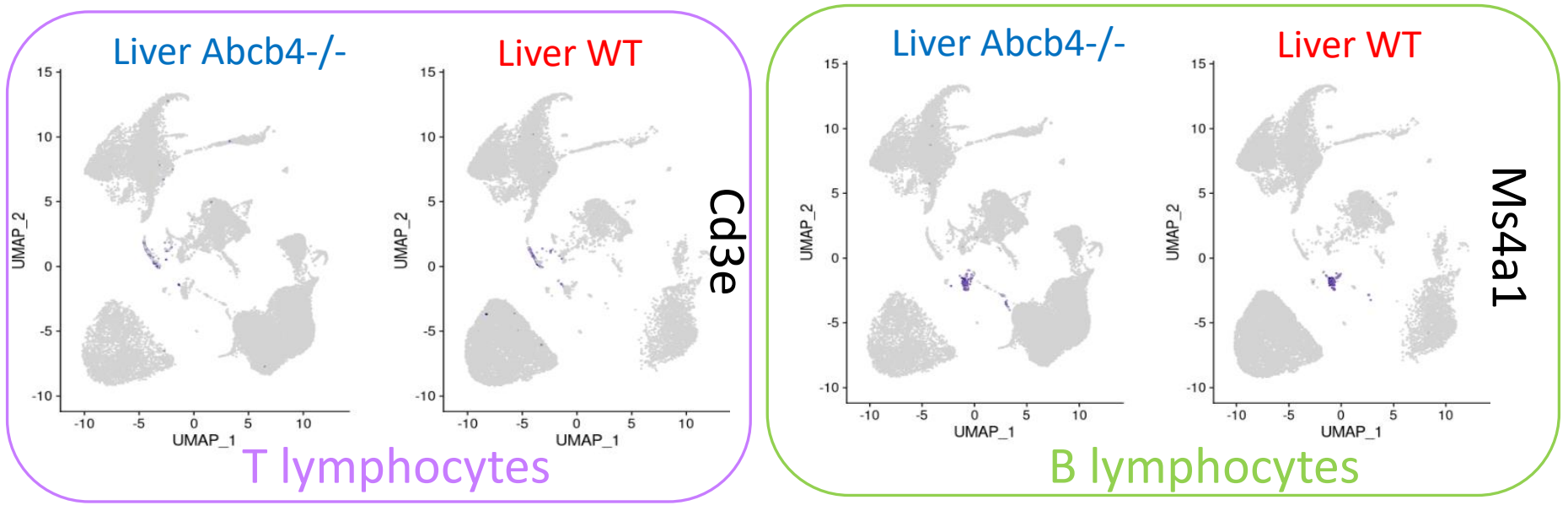

\section{Supplemental Figure 2}




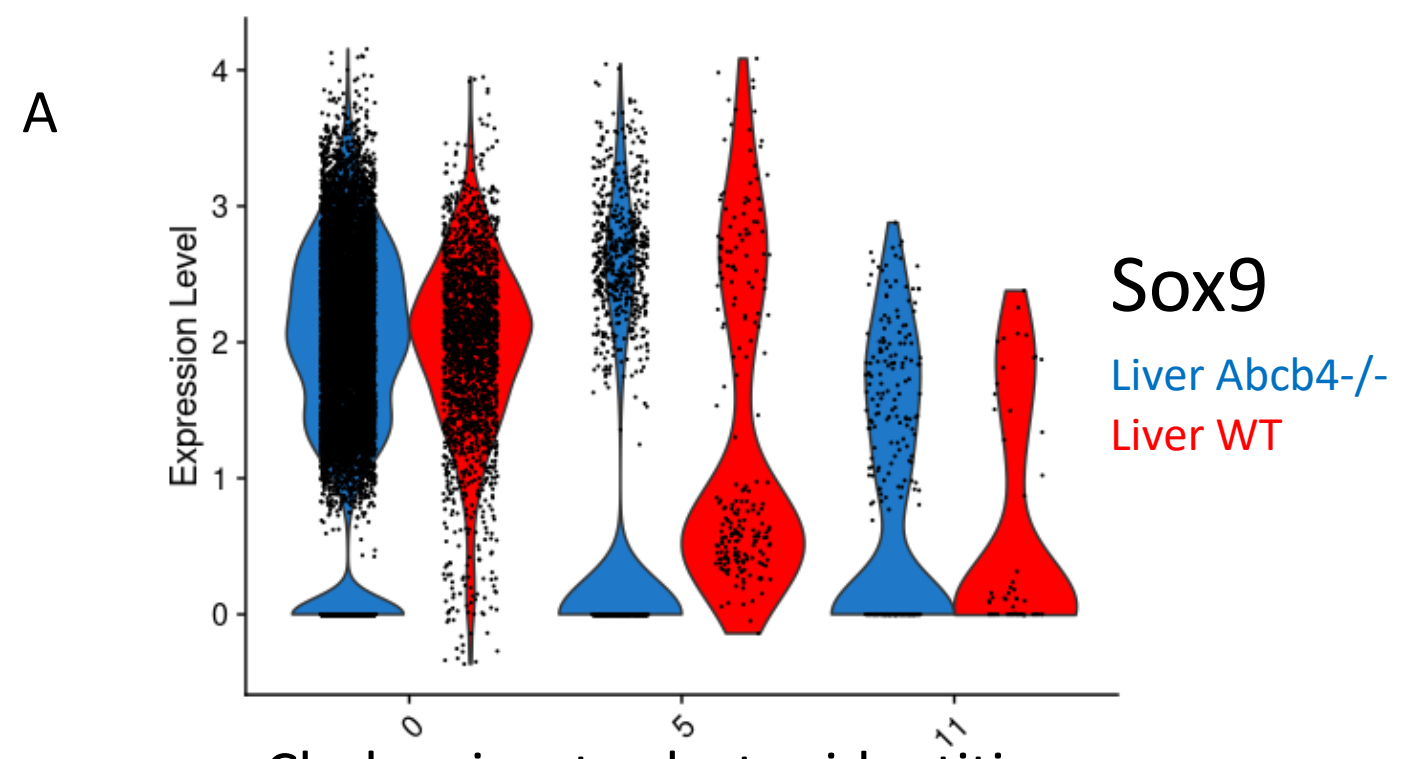

Cholangiocyte cluster identities

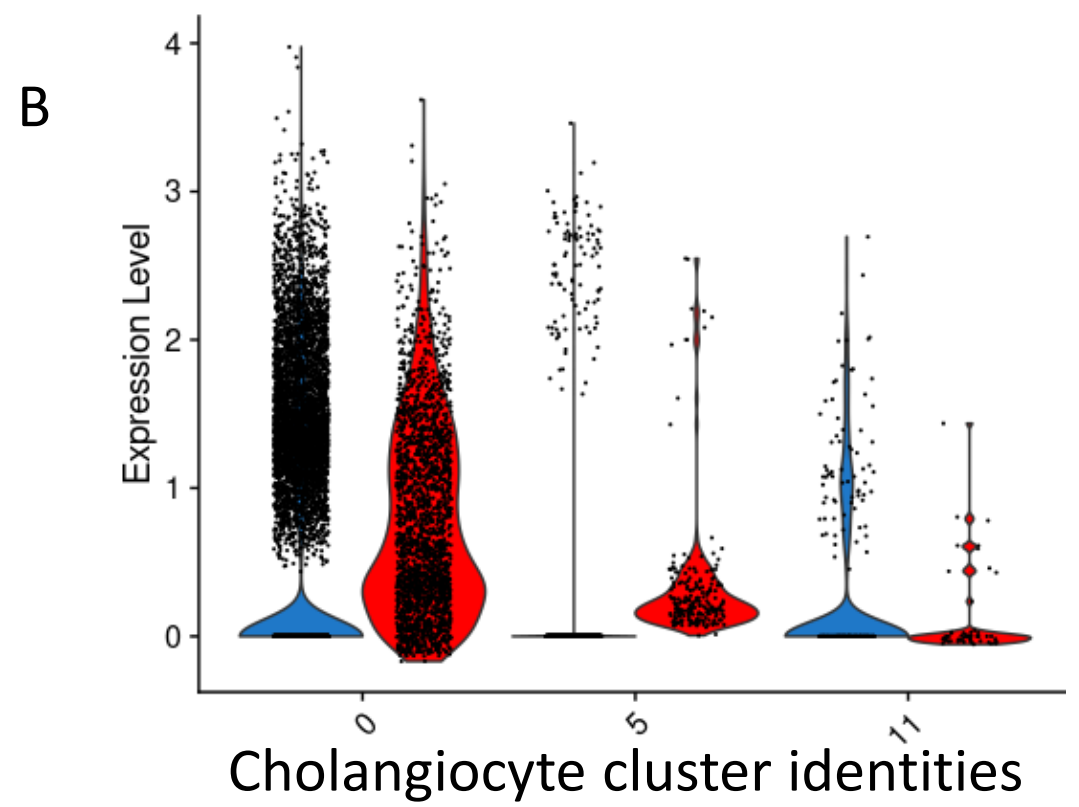

NrOb2

Liver Abcb4-/-

Liver WT

\section{Epcam}

C

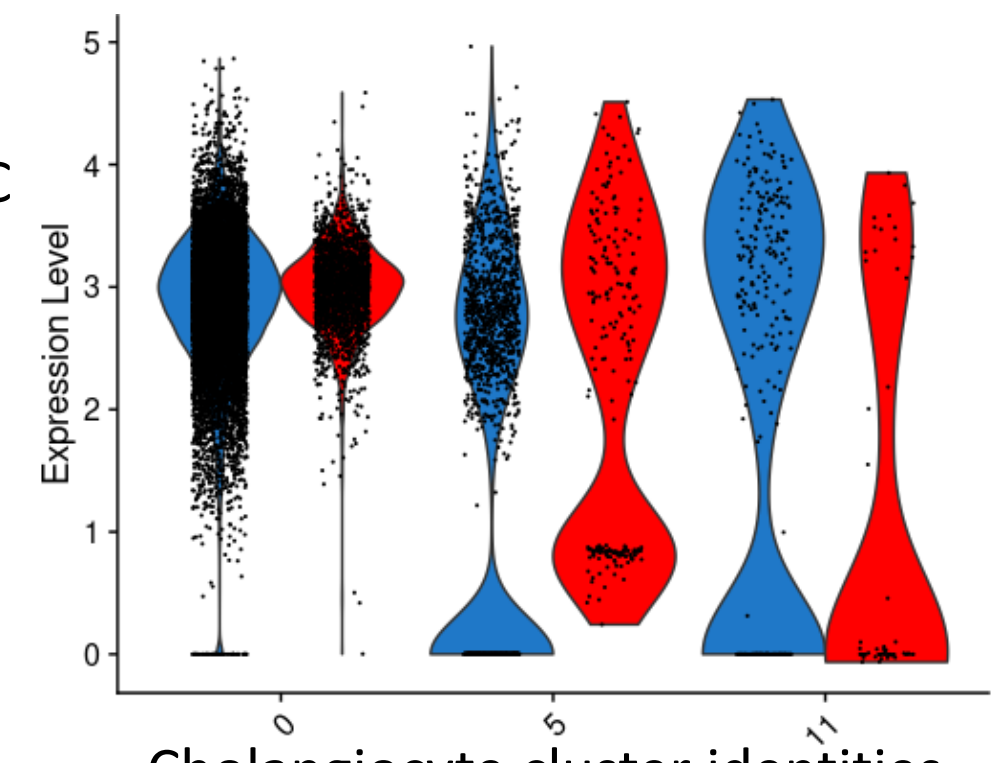

Cholangiocyte cluster identities
Liver Abcb4-/-

Liver WT 
A

T-SNE stratification NrOb2-Sox9
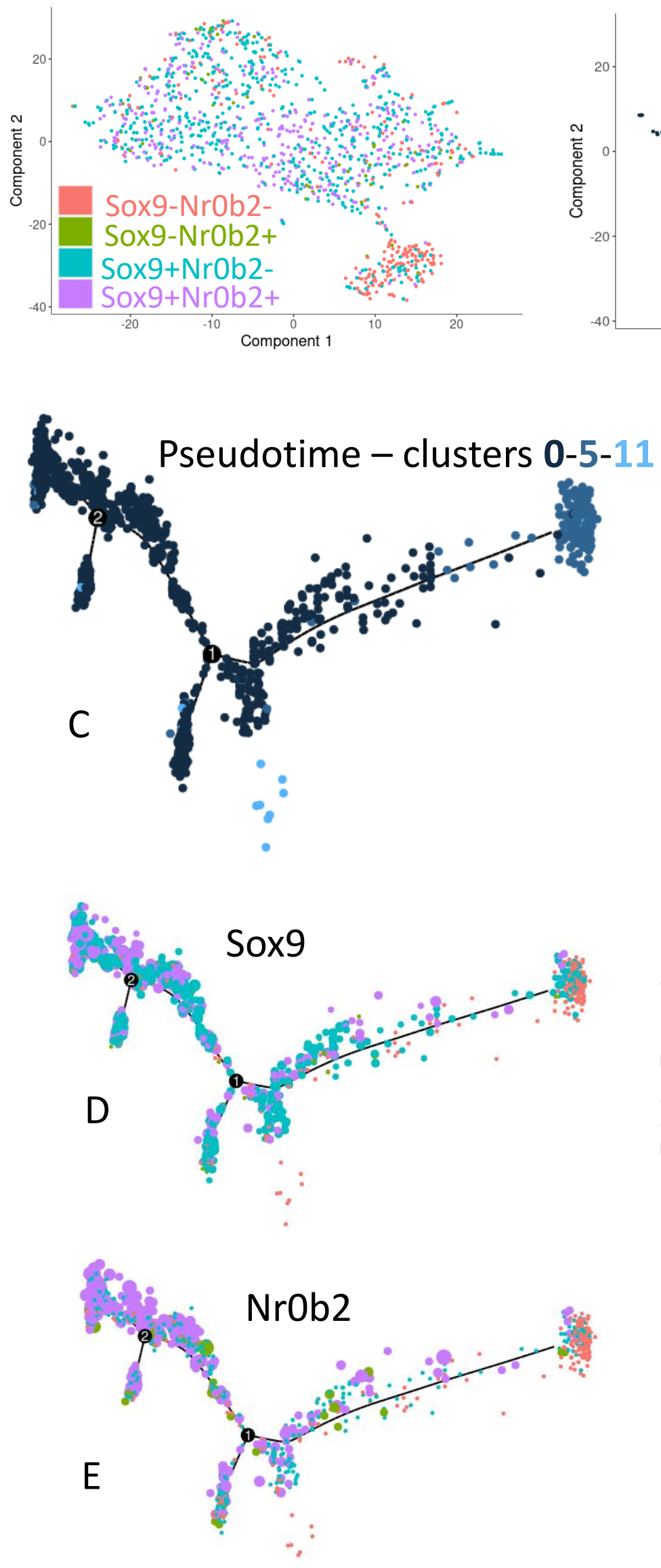

B

T-SNE- cholangiocyte clusters 0-5-11

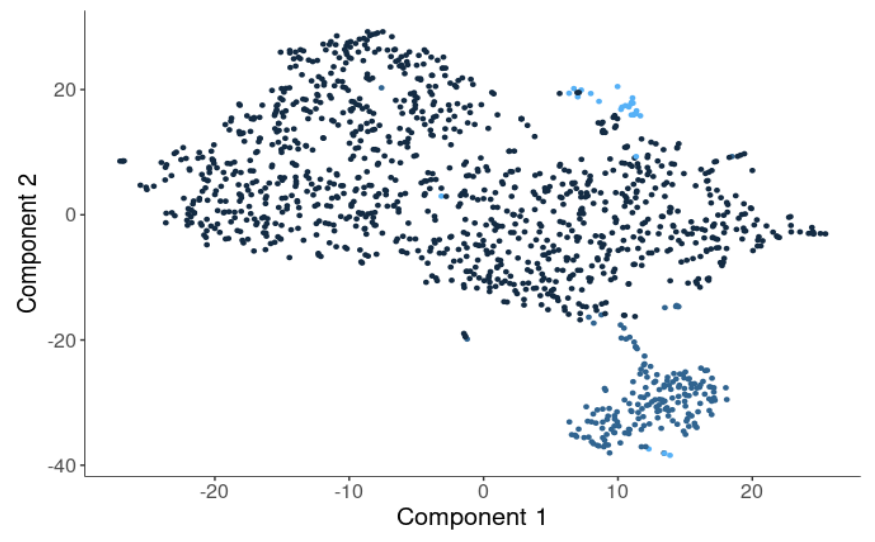

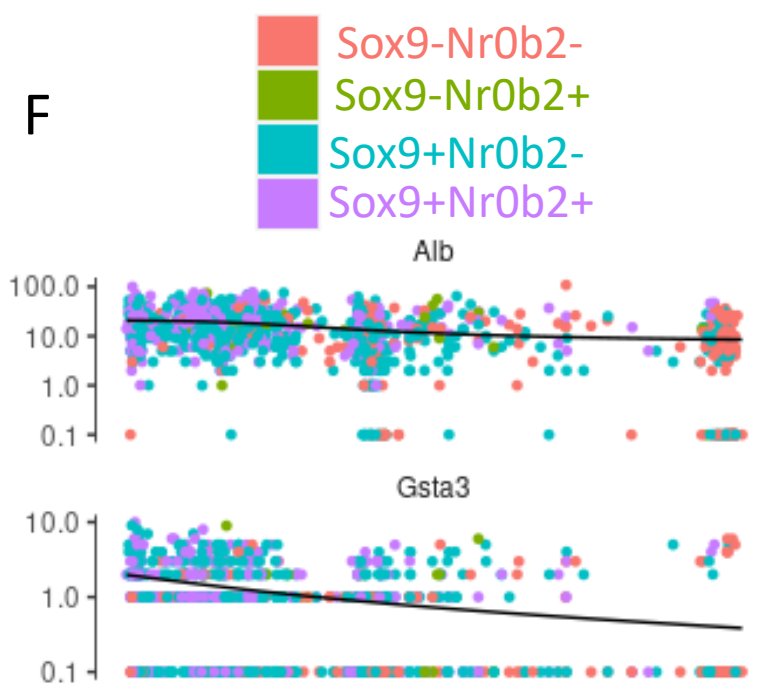

Id2
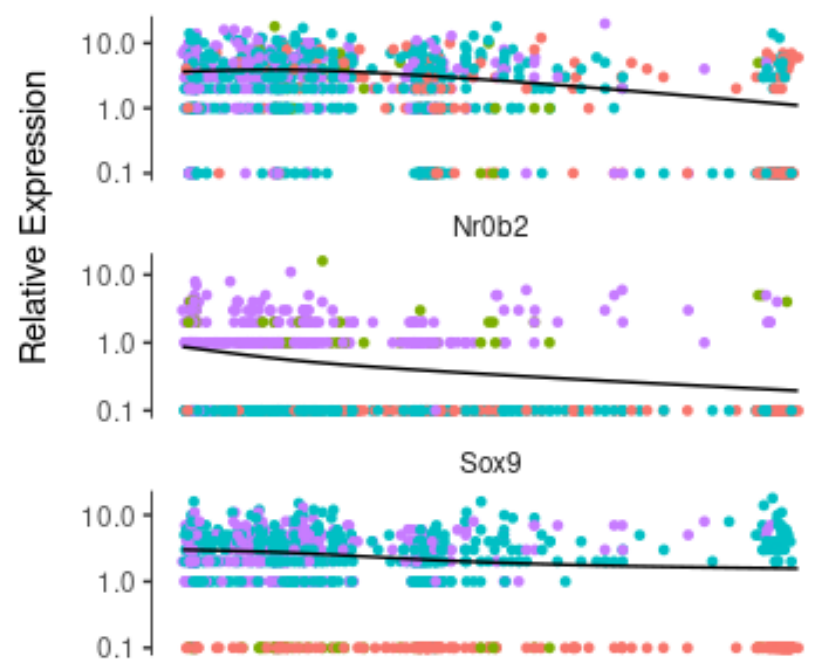

Tmem45a

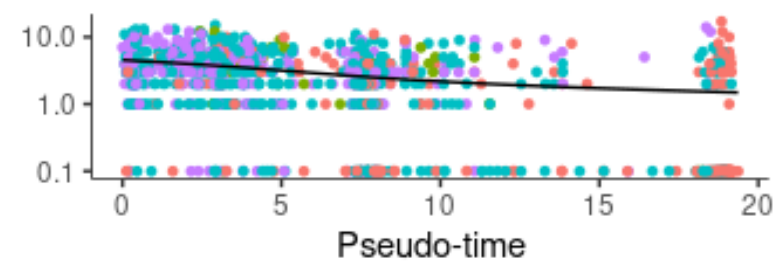




\section{SUPPLEMENTAL MATERIALS}

\section{Supplemental Tables}

Supplemental Table 1: Textmining table of the common 525 ranked genes found in Pubmed with symptom keywords: this table describes the 525 ranked genes obtained by text mining with 'Génie' algorithm against the three MESH terms: biliary inflammation, biliary fibrosis, biliary stasis, respective ranks, $\mathrm{p}$-values and collected PMID identifiers for each gene and each MESH term are presented

Supplemental Table 2: Symptoms related genes found differentially expressed in liver from primary sclerosing cholangitis patients: Differential expressed gene analysis results (logarithm Fold Change, Average Epression and Adjust $\mathrm{p}$-values) are presented with respective machine learning predictive score for supervized sample categories of GSE61256 dataset: PSC versus other liver samples)

Supplemental Table 3: Pavlidis Template Matching for FXR dependency in liver transcriptome: Pavlidis template analysis results to describe FXR regulation dependency of PSC genes in liver samples of GSE54557 dataset. For each gene R-Pearson correlation coefficient and its respective $p$-value are presented in this table.

Supplemental Table 4: Best one hundred genes found to be significant pseudotime trajectory of Abcb4-/- cholangiocytes : Best one hundred ranked genes found as significant on pseudotime cell trajectory of cholangiocytes Abcb4-/- based on alternative expression of NrOb2 and Sox9 in GSE168758 
bioRxiv preprint doi: https://doi.org/10.1101/2021.09.06.459063; this version posted September 6, 2021. The copyright holder for this preprint (which was not certified by peer review) is the author/funder. All rights reserved. No reuse allowed without permission.

\section{Supplemental Table 1}

\begin{tabular}{|c|c|c|c|c|c|c|c|c|c|c|c|c|c|}
\hline gene & $\begin{array}{l}\text { Genel } \\
\text { D }\end{array}$ & $\begin{array}{l}\text { n.total.pu } \\
\text { bmed }\end{array}$ & $\begin{array}{l}\text { Homo } \\
\text { logs }\end{array}$ & $\begin{array}{l}\text { ranking_me } \\
\text { an }\end{array}$ & $\begin{array}{l}\text { Rank.biliary. } \\
\text { fibrosis }\end{array}$ & $\begin{array}{l}\text { Rank.biliary.infla } \\
\text { mmation }\end{array}$ & $\begin{array}{l}\text { Rank.biliary } \\
\text {.stasis }\end{array}$ & $\begin{array}{l}\text { n_pos.biliary. } \\
\text { fibrosis }\end{array}$ & $\begin{array}{l}\text { n_pos.biliary.infl } \\
\text { ammation }\end{array}$ & $\begin{array}{l}\text { n_pos.biliar } \\
\text { y.stasis }\end{array}$ & \begin{tabular}{|l|} 
FDR.biliary.f \\
ibrosis
\end{tabular} & $\begin{array}{l}\text { FDR.biliary.infla } \\
\text { mmation }\end{array}$ & $\begin{array}{l}\text { FDR.biliary } \\
\text {.stasis }\end{array}$ \\
\hline $\mathrm{NR} 1 \mathrm{H} 4$ & 9971 & 486 & 10 & $\begin{array}{l}1.66666666 \\
666667 \\
\end{array}$ & 1 & 3 & 1 & 108 & 153 & 157 & $7.785 \mathrm{e}-144$ & $4.838 \mathrm{e}-210$ & $6.278 \mathrm{e}-212$ \\
\hline ABCB4 & 5244 & 295 & 11 & $\begin{array}{l}5.66666666 \\
666667\end{array}$ & 4 & 10 & 3 & 77 & 82 & 104 & $1.455 \mathrm{e}-118$ & $2.618 \mathrm{e}-127$ & 7.063e-161 \\
\hline $\begin{array}{l}\mathrm{ABCB} 1 \\
1 \\
\end{array}$ & 8647 & 203 & 10 & $\begin{array}{l}8.33333333 \\
333333\end{array}$ & 5 & 18 & 2 & 58 & 62 & 101 & $6.742 \mathrm{e}-98$ & $8.01 \mathrm{e}-106$ & $2.523 \mathrm{e}-176$ \\
\hline TGFB1 & 7040 & 6275 & 9 & 11 & 6 & 8 & 19 & 202 & 339 & 113 & $1.35 \mathrm{e}-79$ & $8.031 \mathrm{e}-138$ & $5.692 \mathrm{e}-40$ \\
\hline IFNL3 & $\begin{array}{r}28261 \\
7 \\
\end{array}$ & 527 & 10 & 12 & 2 & 27 & 7 & 100 & 69 & 65 & $5.873 \mathrm{e}-129$ & $1.062 \mathrm{e}-87$ & $7.138 \mathrm{e}-79$ \\
\hline $\begin{array}{l}\text { PNPLA } \\
3 \\
\end{array}$ & 80339 & 262 & 8 & 17 & 3 & 42 & 6 & 80 & 45 & 53 & $3.532 \mathrm{e}-128$ & $8.692 \mathrm{e}-70$ & $1.284 \mathrm{e}-79$ \\
\hline IL6 & 3569 & 6198 & 7 & $\begin{array}{l}17.33333333 \\
333333\end{array}$ & 15 & 1 & 36 & 117 & 551 & 79 & 7.473e-45 & $1.318 \mathrm{e}-230$ & $9.478 \mathrm{e}-27$ \\
\hline TLR4 & 7099 & 4840 & 11 & $\begin{array}{l}18.33333333 \\
333333 \\
\end{array}$ & 18 & 4 & 33 & 96 & 389 & 69 & $7.342 \mathrm{e}-43$ & $1.276 \mathrm{e}-188$ & $2.147 \mathrm{e}-27$ \\
\hline $\begin{array}{l}\text { GPBAR } \\
1\end{array}$ & $\begin{array}{r}15130 \\
6 \\
\end{array}$ & 114 & 7 & 20 & 10 & 38 & 12 & 29 & 38 & 29 & $3.938 \mathrm{e}-54$ & $1.874 \mathrm{e}-73$ & $5.339 \mathrm{e}-52$ \\
\hline IL17A & 3605 & 2099 & 8 & $\begin{array}{l}21.33333333 \\
333333 \\
\end{array}$ & 14 & 6 & 44 & 64 & 225 & 42 & $6.968 \mathrm{e}-45$ & $1.34 \mathrm{e}-170$ & $2.905 \mathrm{e}-26$ \\
\hline TNF & 7124 & 8701 & 8 & $\begin{array}{l}21.6666666 \\
666667 \\
\end{array}$ & 17 & 2 & 46 & 141 & 656 & 95 & $6.731 \mathrm{e}-43$ & $4.088 \mathrm{e}-218$ & $2.117 \mathrm{e}-25$ \\
\hline $\begin{array}{l}\text { HMOX } \\
1\end{array}$ & 3162 & 1990 & 10 & $\begin{array}{l}22.6666666 \\
666667\end{array}$ & 24 & 26 & 18 & 56 & 122 & 61 & $4.269 \mathrm{e}-40$ & $2.293 \mathrm{e}-92$ & $3.275 \mathrm{e}-41$ \\
\hline $\begin{array}{l}\text { ATP8B } \\
1 \\
\end{array}$ & 5205 & 88 & 10 & 23 & 8 & 57 & 4 & 32 & 28 & 46 & $1.662 \mathrm{e}-64$ & $1.792 \mathrm{e}-56$ & $8.701 \mathrm{e}-94$ \\
\hline NFE2L2 & 4780 & 2172 & 9 & $\begin{array}{l}24.6666666 \\
666667\end{array}$ & 26 & 19 & 29 & 56 & 141 & 50 & $2.393 \mathrm{e}-38$ & $7.63 \mathrm{e}-103$ & $2.115 \mathrm{e}-31$ \\
\hline PPARA & 5465 & 1457 & 11 & $\begin{array}{l}26.66666666 \\
666667\end{array}$ & 28 & 39 & 13 & 43 & 85 & 56 & $4.818 \mathrm{e}-35$ & $3.185 \mathrm{e}-73$ & $9.144 \mathrm{e}-44$ \\
\hline HNF4A & 3172 & 832 & 11 & 29 & 21 & 52 & 14 & 41 & 55 & 44 & $2.884 \mathrm{e}-42$ & $1.805 e-58$ & $5.653 \mathrm{e}-43$ \\
\hline KRT19 & 3880 & 403 & 8 & 30 & 11 & 56 & 23 & 39 & 42 & 29 & $9.56 \mathrm{e}-52$ & $8.678 e-57$ & $1.599 \mathrm{e}-35$ \\
\hline CFTR & 1080 & 1992 & 10 & $\begin{array}{l}30.33333333 \\
333333 \\
\end{array}$ & 19 & 40 & 32 & 59 & 97 & 43 & $2.537 \mathrm{e}-42$ & $1.324 \mathrm{e}-72$ & $1.13 \mathrm{e}-27$ \\
\hline HFE & 3077 & 1015 & 7 & $\begin{array}{l}30.6666666 \\
666667\end{array}$ & 7 & 76 & 9 & 71 & 49 & 61 & $5.66 \mathrm{e}-70$ & $7.329 \mathrm{e}-48$ & $8.847 \mathrm{e}-57$ \\
\hline CXCL10 & 3627 & 820 & 7 & 31 & 22 & 36 & 35 & 39 & 69 & 29 & $2.846 \mathrm{e}-40$ & $1.139 \mathrm{e}-74$ & $4.507 \mathrm{e}-27$ \\
\hline CTGF & 1490 & 939 & 9 & 32 & 12 & 45 & 39 & 50 & 64 & 30 & $1.096 \mathrm{e}-49$ & $1.983 \mathrm{e}-65$ & $1.42 \mathrm{e}-26$ \\
\hline SPP1 & 6696 & 1677 & 7 & $\begin{array}{l}32.66666666 \\
666667\end{array}$ & 23 & 34 & 41 & 52 & 97 & 38 & $2.93 \mathrm{e}-40$ & $7.601 \mathrm{e}-79$ & $2.141 \mathrm{e}-26$ \\
\hline HGF & 3082 & 1121 & 10 & $\begin{array}{l}37.3333333 \\
333333 \\
\end{array}$ & 20 & 58 & 34 & 46 & 58 & 33 & $2.584 \mathrm{e}-42$ & 7.407e-55 & $2.58 \mathrm{e}-27$ \\
\hline AFP & 174 & 546 & 8 & $\begin{array}{l}38.33333333 \\
333333 \\
\end{array}$ & 9 & 82 & 24 & 51 & 38 & 31 & $2.869 \mathrm{e}-62$ & $3.469 \mathrm{e}-46$ & $2.538 \mathrm{e}-34$ \\
\hline SТАТЗ & 6774 & 3858 & 10 & 41 & 44 & 14 & 65 & 57 & 208 & 42 & $3.088 \mathrm{e}-28$ & $1.073 \mathrm{e}-112$ & $6.706 \mathrm{e}-18$ \\
\hline $\begin{array}{l}\text { TNFRSF } \\
1 \mathrm{~A}\end{array}$ & 7132 & 1812 & 10 & 44 & 55 & 9 & 68 & 36 & 165 & 26 & $4.091 \mathrm{e}-26$ & $2.034 \mathrm{e}-132$ & $3.815 \mathrm{e}-16$ \\
\hline CRP & 1401 & 2117 & 29 & $\begin{array}{l}44.3333333 \\
333333\end{array}$ & 31 & 47 & 55 & 48 & 88 & 33 & $7.068 \mathrm{e}-33$ & $1.43 \mathrm{e}-63$ & $1.183 \mathrm{e}-19$ \\
\hline $\mathrm{NR} 1 \mathrm{H} 3$ & 10062 & 684 & 9 & 46 & 67 & 41 & 30 & 22 & 62 & 30 & $2.516 \mathrm{e}-23$ & $1.918 \mathrm{e}-71$ & $2.504 \mathrm{e}-30$ \\
\hline NR112 & 8856 & 551 & 10 & 46 & 34 & 89 & 15 & 28 & 37 & 38 & $1.032 \mathrm{e}-32$ & $8.953 \mathrm{e}-45$ & $6.609 \mathrm{e}-43$ \\
\hline IL22 & 50616 & 475 & 10 & $\begin{array}{l}47.6666666 \\
666667 \\
\end{array}$ & 38 & 23 & 82 & 25 & 71 & 14 & $1.799 \mathrm{e}-30$ & $1.242 \mathrm{e}-93$ & $1.848 \mathrm{e}-14$ \\
\hline KRT18 & 3875 & 449 & 10 & 48 & 16 & 102 & 26 & 34 & 31 & 29 & $4.062 \mathrm{e}-43$ & $9.082 \mathrm{e}-40$ & $3.146 \mathrm{e}-34$ \\
\hline IL10 & 3586 & 4840 & 10 & $\begin{array}{l}48.6666666 \\
666667 \\
\end{array}$ & 40 & 5 & 101 & 68 & 377 & 35 & $1.365 \mathrm{e}-29$ & $1.34 \mathrm{e}-182$ & $4.496 \mathrm{e}-12$ \\
\hline NOS2 & 4843 & 2636 & 12 & $\begin{array}{l}48.66666666 \\
666667\end{array}$ & 57 & 30 & 59 & 42 & 125 & 36 & $2.348 \mathrm{e}-25$ & $5.07 e-82$ & $4.009 \mathrm{e}-19$ \\
\hline HNF1B & 6928 & 446 & 10 & $\begin{array}{l}50.3333333 \\
333333 \\
\end{array}$ & 25 & 101 & 25 & 31 & 31 & 29 & $3.015 \mathrm{e}-39$ & $7.463 e-40$ & $2.701 \mathrm{e}-34$ \\
\hline NLRP3 & $\begin{array}{r}11454 \\
8 \\
\end{array}$ & 1166 & 24 & $\begin{array}{l}51.6666666 \\
666667 \\
\end{array}$ & 65 & 12 & 78 & 27 & 120 & 20 & $1.863 \mathrm{e}-23$ & $1.076 \mathrm{e}-115$ & $6.692 \mathrm{e}-15$ \\
\hline $\begin{array}{l}\text { CYP7A } \\
1\end{array}$ & 1581 & 237 & 10 & $\begin{array}{l}51.6666666 \\
666667\end{array}$ & 66 & 79 & 10 & 16 & 30 & 37 & $2.027 \mathrm{e}-23$ & $9.997 \mathrm{e}-47$ & $3.093 e-55$ \\
\hline FAS & 355 & 2660 & 10 & $\begin{array}{l}54.3333333 \\
333333 \\
\end{array}$ & 42 & 65 & 56 & 48 & 82 & 37 & $3.898 \mathrm{e}-29$ & $2.025 e-52$ & $1.224 \mathrm{e}-19$ \\
\hline LCN2 & 3934 & 811 & 7 & $\begin{array}{l}55.33333333 \\
333333 \\
\end{array}$ & 33 & 84 & 49 & 32 & 43 & 25 & $1.037 \mathrm{e}-32$ & $1.429 \mathrm{e}-45$ & $5.63 \mathrm{e}-23$ \\
\hline $\mathrm{ABCC} 2$ & 1244 & 479 & 13 & $\begin{array}{l}55.33333333 \\
333333\end{array}$ & 30 & 128 & 8 & 28 & 28 & 50 & $2.54 \mathrm{e}-34$ & $5.953 e-35$ & $4.028 \mathrm{e}-61$ \\
\hline PPARG & 5468 & 3417 & 9 & 56 & 73 & 44 & 51 & 42 & 116 & 47 & $8.096 \mathrm{e}-22$ & $1.115 \mathrm{e}-65$ & $3.058 \mathrm{e}-22$ \\
\hline ALB & 213 & 855 & 9 & $\begin{array}{l}57.3333333 \\
333333 \\
\end{array}$ & 27 & 107 & 38 & 37 & 38 & 29 & $1.797 \mathrm{e}-37$ & $3.52 \mathrm{e}-39$ & $1.285 \mathrm{e}-26$ \\
\hline SMAD3 & 4088 & 1507 & 12 & $\begin{array}{l}59.3333333 \\
333333\end{array}$ & 32 & 66 & 80 & 41 & 62 & 22 & $8.859 \mathrm{e}-33$ & $9.906 e-52$ & $1.251 \mathrm{e}-14$ \\
\hline
\end{tabular}




\begin{tabular}{|c|c|c|c|c|c|c|c|c|c|c|c|c|c|}
\hline $\begin{array}{l}\text { SERPIN } \\
\text { A1 }\end{array}$ & 5265 & 633 & 20 & 60 & 29 & 124 & 27 & 31 & 31 & 32 & $1.051 \mathrm{e}-34$ & $2.458 \mathrm{e}-35$ & $1.171 \mathrm{e}-33$ \\
\hline FGF19 & 9965 & 239 & 10 & $\begin{array}{l}61.3333333 \\
333333\end{array}$ & 37 & 125 & 22 & 21 & 23 & 26 & $2.533 \mathrm{e}-31$ & $3.706 \mathrm{e}-35$ & $2.871 \mathrm{e}-37$ \\
\hline IFNG & 3458 & 4209 & 7 & 62 & 59 & 16 & 111 & 52 & 212 & 31 & $3.346 \mathrm{e}-24$ & $3.914 \mathrm{e}-109$ & $1.484 \mathrm{e}-11$ \\
\hline $\begin{array}{l}\text { ADIPO } \\
Q\end{array}$ & 9370 & 2651 & 10 & $\begin{array}{l}62.3333333 \\
333333\end{array}$ & 56 & 68 & 63 & 43 & 79 & 35 & $6.114 \mathrm{e}-26$ & $1.745 e-50$ & $1.961 \mathrm{e}-18$ \\
\hline $\begin{array}{l}\text { ONECU } \\
\text { T1 }\end{array}$ & 3175 & 193 & 8 & $\begin{array}{l}62.6666666 \\
666667\end{array}$ & 36 & 109 & 43 & 20 & 24 & 18 & $1.448 \mathrm{e}-31$ & $4.602 \mathrm{e}-39$ & $2.526 \mathrm{e}-26$ \\
\hline $\begin{array}{l}\text { SLC10A } \\
1\end{array}$ & 6554 & 111 & 9 & 67 & 53 & 137 & 11 & 15 & 18 & 29 & $8.911 \mathrm{e}-27$ & $3.412 \mathrm{e}-33$ & $2.415 e-52$ \\
\hline MMP9 & 4318 & 4106 & 12 & $\begin{array}{l}67.3333333 \\
333333\end{array}$ & 49 & 55 & 98 & 57 & 115 & 32 & $3.536 \mathrm{e}-27$ & $2.165 \mathrm{e}-58$ & $3.148 \mathrm{e}-12$ \\
\hline CD14 & 929 & 961 & 7 & 74 & 75 & 53 & 94 & 23 & 58 & 16 & $2.189 \mathrm{e}-21$ & $1.955 e-58$ & $1.7 \mathrm{e}-12$ \\
\hline TIMP1 & 7076 & 945 & 6 & 74 & 39 & 93 & 90 & 31 & 43 & 17 & $9.862 \mathrm{e}-30$ & $6.334 \mathrm{e}-43$ & $1.391 \mathrm{e}-13$ \\
\hline SREBF1 & 6720 & 836 & 12 & $\begin{array}{l}74.3333333 \\
333333\end{array}$ & 63 & 113 & 47 & 24 & 36 & 27 & $1.047 \mathrm{e}-23$ & $2.58 \mathrm{e}-37$ & $8.283 \mathrm{e}-25$ \\
\hline MMP2 & 4313 & 2608 & 10 & $\begin{array}{l}75.3333333 \\
333333 \\
\end{array}$ & 46 & 97 & 83 & 45 & 65 & 28 & $1.853 \mathrm{e}-27$ & $1.854 \mathrm{e}-41$ & $3.359 \mathrm{e}-14$ \\
\hline IL1B & 3553 & 4025 & 8 & $\begin{array}{l}75.6666666 \\
666667 \\
\end{array}$ & 84 & 7 & 136 & 43 & 295 & 27 & $3.072 \mathrm{e}-20$ & $2.541 \mathrm{e}-158$ & $4.81 \mathrm{e}-10$ \\
\hline CXCR3 & 2833 & 507 & 9 & $\begin{array}{l}76.3333333 \\
333333\end{array}$ & 48 & 73 & 108 & 23 & 39 & 12 & $3.037 \mathrm{e}-27$ & 1.116e-48 & $1.014 \mathrm{e}-11$ \\
\hline LEPR & 3953 & 2229 & 10 & $\begin{array}{l}78.3333333 \\
333333 \\
\end{array}$ & 70 & 69 & 96 & 34 & 72 & 23 & $4.802 \mathrm{e}-22$ & $3.904 \mathrm{e}-50$ & $2.706 \mathrm{e}-12$ \\
\hline LDLR & 3949 & 1935 & 9 & 79 & 116 & 60 & 61 & 24 & 72 & 30 & $3.442 \mathrm{e}-16$ & $8.426 \mathrm{e}-54$ & $1.727 \mathrm{e}-18$ \\
\hline HAMP & 57817 & 365 & 5 & $\begin{array}{l}80.3333333 \\
333333\end{array}$ & 72 & 103 & 66 & 17 & 29 & 15 & $6.472 \mathrm{e}-22$ & $1.311 \mathrm{e}-39$ & $2.632 \mathrm{e}-17$ \\
\hline JAG1 & 182 & 1196 & 12 & $\begin{array}{l}80.6666666 \\
666667 \\
\end{array}$ & 51 & 151 & 40 & 31 & 35 & 33 & $6.015 \mathrm{e}-27$ & $2.696 \mathrm{e}-31$ & $1.504 \mathrm{e}-26$ \\
\hline NR113 & 9970 & 281 & 8 & $\begin{array}{l}82.3333333 \\
333333 \\
\end{array}$ & 79 & 126 & 42 & 15 & 24 & 20 & $9.475 \mathrm{e}-21$ & $4.278 \mathrm{e}-35$ & $2.115 \mathrm{e}-26$ \\
\hline PDCD1 & 5133 & 924 & 9 & 85 & 52 & 63 & 140 & 28 & 52 & 13 & $6.332 \mathrm{e}-27$ & $6.571 \mathrm{e}-53$ & $6.449 \mathrm{e}-10$ \\
\hline NROB2 & 8431 & 259 & 10 & $\begin{array}{l}85.6666666 \\
666667 \\
\end{array}$ & 76 & 165 & 16 & 15 & 20 & 30 & $2.904 \mathrm{e}-21$ & $1.292 \mathrm{e}-29$ & $1.322 \mathrm{e}-42$ \\
\hline LGALS3 & 3958 & 923 & 10 & 86 & 45 & 108 & 105 & 29 & 39 & 15 & $6.047 \mathrm{e}-28$ & $4.444 \mathrm{e}-39$ & $8.58 \mathrm{e}-12$ \\
\hline SIRT1 & 23411 & 1802 & 10 & $\begin{array}{l}87.3333333 \\
333333\end{array}$ & 106 & 94 & 62 & 24 & 56 & 29 & $8.926 \mathrm{e}-17$ & 9.553e-43 & $1.723 \mathrm{e}-18$ \\
\hline LEP & 3952 & 3609 & 8 & $\begin{array}{l}87.6666666 \\
666667 \\
\end{array}$ & 94 & 92 & 77 & 37 & 81 & 35 & $2.41 \mathrm{e}-18$ & $1.353 \mathrm{e}-43$ & $5.221 \mathrm{e}-15$ \\
\hline $\begin{array}{l}\text { NOTCH } \\
2 \\
\end{array}$ & 4853 & 669 & 10 & $\begin{array}{l}87.6666666 \\
666667\end{array}$ & 69 & 130 & 64 & 21 & 31 & 19 & $2.417 \mathrm{e}-22$ & $1.217 \mathrm{e}-34$ & $4.353 \mathrm{e}-18$ \\
\hline CHI3L1 & 1116 & 409 & 9 & $\begin{array}{l}88.3333333 \\
333333 \\
\end{array}$ & 35 & 117 & 113 & 25 & 28 & 11 & $4.92 \mathrm{e}-32$ & $8.239 \mathrm{e}-37$ & $1.713 \mathrm{e}-11$ \\
\hline NFKB1 & 4790 & 4419 & 9 & $\begin{array}{l}88.6666666 \\
666667\end{array}$ & 98 & 17 & 151 & 40 & 212 & 27 & $1.452 \mathrm{e}-17$ & $6.239 \mathrm{e}-106$ & $\begin{array}{l}0.0000000 \\
02375\end{array}$ \\
\hline $\begin{array}{l}\text { SERPIN } \\
\text { E1 }\end{array}$ & 5054 & 1808 & 12 & 89 & 62 & 110 & 95 & 33 & 51 & 21 & $9.107 e-24$ & $1.178 \mathrm{e}-38$ & $1.815 \mathrm{e}-12$ \\
\hline $\begin{array}{l}\text { COL1A } \\
1 \\
\end{array}$ & 1277 & 1616 & 9 & 89 & 41 & 142 & 84 & 38 & 41 & 22 & $3.107 \mathrm{e}-29$ & $2.441 \mathrm{e}-32$ & $4.425 \mathrm{e}-14$ \\
\hline CCL13 & 6357 & 1262 & 5 & $\begin{array}{l}89.33333333 \\
333333 \\
\end{array}$ & 125 & 24 & 119 & 19 & 101 & 16 & $2.16 \mathrm{e}-15$ & $1.887 \mathrm{e}-93$ & $6.502 \mathrm{e}-11$ \\
\hline $\begin{array}{l}\text { HMGB } \\
1\end{array}$ & 3146 & 904 & 8 & $\begin{array}{l}89.6666666 \\
666667 \\
\end{array}$ & 60 & 32 & 177 & 25 & 76 & 11 & $5.299 \mathrm{e}-24$ & $1.431 \mathrm{e}-79$ & $\begin{array}{l}0.0000000 \\
3828\end{array}$ \\
\hline IL18 & 3606 & 1496 & 7 & 92 & 99 & 21 & 156 & 23 & 112 & 15 & $1.533 \mathrm{e}-17$ & $1.452 \mathrm{e}-96$ & $\begin{array}{l}0.0000000 \\
03399\end{array}$ \\
\hline ICAM1 & 3383 & 1864 & 7 & $\begin{array}{l}97.33333333 \\
333333 \\
\end{array}$ & 121 & 59 & 112 & 23 & 72 & 20 & $9.59 \mathrm{e}-16$ & $8.539 \mathrm{e}-55$ & $1.473 \mathrm{e}-11$ \\
\hline CCR5 & 1234 & 1824 & 11 & 99 & 86 & 83 & 128 & 28 & 60 & 18 & $8.811 \mathrm{e}-20$ & $1.055 \mathrm{e}-45$ & $2.896 \mathrm{e}-10$ \\
\hline CCL5 & 6352 & 958 & 8 & $\begin{array}{l}100.3333333 \\
333333\end{array}$ & 74 & 43 & 184 & 23 & 67 & 11 & $2.076 \mathrm{e}-21$ & $4.027 \mathrm{e}-68$ & $\begin{array}{l}0.0000000 \\
6536\end{array}$ \\
\hline KRT8 & 3856 & 633 & 11 & 102 & 54 & 199 & 53 & 24 & 23 & 22 & $2.218 \mathrm{e}-26$ & $1.005 \mathrm{e}-25$ & $5.607 \mathrm{e}-22$ \\
\hline $\begin{array}{l}\text { TNFRSF } \\
1 \mathrm{~B}\end{array}$ & 7133 & 1050 & 8 & $\begin{array}{l}103.333333 \\
333333\end{array}$ & 107 & 35 & 168 & 19 & 78 & 12 & $1.089 \mathrm{e}-16$ & $5.251 \mathrm{e}-77$ & $\begin{array}{l}0.0000000 \\
2066\end{array}$ \\
\hline TLR3 & 7098 & 1093 & 12 & 104 & 112 & 80 & 120 & 19 & 49 & 15 & $2.077 \mathrm{e}-16$ & $2.004 \mathrm{e}-46$ & $7.33 \mathrm{e}-11$ \\
\hline TLR2 & 7097 & 2916 & 13 & $\begin{array}{l}106.333333 \\
333333 \\
\end{array}$ & 127 & 13 & 179 & 28 & 179 & 19 & $2.528 \mathrm{e}-15$ & $5.61 \mathrm{e}-113$ & $\begin{array}{l}0.0000000 \\
4105 \\
\end{array}$ \\
\hline VEGFA & 7422 & 6064 & 7 & $\begin{array}{l}106.666666 \\
666667\end{array}$ & 50 & 96 & 174 & 72 & 106 & 30 & $4.766 \mathrm{e}-27$ & $1.096 \mathrm{e}-41$ & $\begin{array}{l}0.0000000 \\
3062\end{array}$ \\
\hline $\mathrm{CCL} 2$ & 6347 & 1332 & 7 & 107 & 91 & 54 & 176 & 23 & 66 & 13 & $1.595 \mathrm{e}-18$ & $2.18 \mathrm{e}-58$ & $\begin{array}{l}0.0000000 \\
3428\end{array}$ \\
\hline PTGS2 & 5743 & 3482 & 10 & $\begin{array}{l}107.333333 \\
333333\end{array}$ & 95 & 49 & 178 & 36 & 109 & 21 & $3.416 \mathrm{e}-18$ & $7.622 \mathrm{e}-61$ & $\begin{array}{l}0.0000000 \\
4118\end{array}$ \\
\hline PKHD1 & 5314 & 103 & 8 & $\begin{array}{l}107.333333 \\
333333\end{array}$ & 47 & 221 & 54 & 15 & 13 & 12 & $3.056 \mathrm{e}-27$ & $7.226 \mathrm{e}-24$ & $1.048 \mathrm{e}-19$ \\
\hline CXCL8 & 3576 & 2063 & 5 & 108 & 68 & 20 & 236 & 34 & 135 & 13 & $5.628 \mathrm{e}-23$ & $7.742 \mathrm{e}-101$ & $\begin{array}{l}0.0000028 \\
77\end{array}$ \\
\hline MIF & 4282 & 975 & 8 & $\begin{array}{l}109.666666 \\
666667\end{array}$ & 128 & 15 & 186 & 17 & 109 & 11 & $2.97 \mathrm{e}-15$ & $1.065 \mathrm{e}-112$ & $\begin{array}{l}0.0000000 \\
7688\end{array}$ \\
\hline CD163 & 9332 & 222 & 7 & $\begin{array}{l}110.333333 \\
333333\end{array}$ & 82 & 106 & 143 & 14 & 25 & 8 & $1.211 \mathrm{e}-20$ & $3.136 \mathrm{e}-39$ & $9.321 \mathrm{e}-10$ \\
\hline
\end{tabular}




\begin{tabular}{|c|c|c|c|c|c|c|c|c|c|c|c|c|c|}
\hline SMAD7 & 4092 & 532 & 10 & $\begin{array}{l}111 . .6666666 \\
666667\end{array}$ & 83 & 127 & 125 & 18 & 29 & 11 & $1.485 \mathrm{e}-20$ & $5.273 \mathrm{e}-35$ & $2.453 \mathrm{e}-10$ \\
\hline MBL2 & 4153 & 845 & 13 & \begin{tabular}{|l|}
112.6666666 \\
666667 \\
\end{tabular} & 43 & 203 & 92 & 29 & 25 & 16 & 5.815e-29 & $3.259 \mathrm{e}-25$ & $2.662 \mathrm{e}-13$ \\
\hline CCR2 & $\begin{array}{r}72923 \\
0 \\
\end{array}$ & 1068 & 10 & $\begin{array}{r}114 \\
\end{array}$ & 138 & 50 & 154 & 17 & 61 & 13 & \begin{tabular}{|l|l|} 
& $1.128 \mathrm{e}-14$ \\
\end{tabular} & 5.149e-59 & \begin{tabular}{|l|l}
0.0000000 \\
0317 \\
\end{tabular} \\
\hline ABCC3 & 8714 & 247 & 26 & \begin{tabular}{|l|}
120.333333 \\
333333
\end{tabular} & 93 & 251 & 17 & 13 & 15 & 29 & $1.954 \mathrm{e}-18$ & $4.307 \mathrm{e}-22$ & 1.214e- 41 \\
\hline MET & 4233 & 1475 & 10 & $\begin{array}{l}124.666666 \\
666667 \\
6\end{array}$ & 92 & 145 & 137 & 24 & 39 & 16 & 1.64e-18 & $5.444 \mathrm{e}-32$ & $4.881 \mathrm{e}-10$ \\
\hline 1133 & 90865 & 637 & 6 & $\begin{array}{l}126.3333333 \\
333333 \\
\end{array}$ & 139 & 11 & 229 & 14 & 97 & 8 & 1.33e-14 & $2.894 \mathrm{e}-117$ & $\begin{array}{l}0.0000019 \\
03 \\
\end{array}$ \\
\hline FASLG & 356 & 1399 & 10 & $\begin{array}{l}126.333333 \\
333333 \\
\end{array}$ & 113 & 140 & 126 & 21 & 39 & 16 & $2.178 \mathrm{e}-16$ & $9.092 \mathrm{e}-33$ & $2.568 \mathrm{e}-10$ \\
\hline $\begin{array}{l}\text { TGFBR } \\
2 \\
\end{array}$ & 7048 & 983 & 10 & 129 & 77 & 163 & 147 & 23 & 31 & 13 & \begin{tabular}{|l}
$3.44 \mathrm{e}-21$ \\
\end{tabular} & $7.308 \mathrm{e}-30$ & $\begin{array}{l}0.00000000 \\
01268 \\
\end{array}$ \\
\hline APOE & 348 & 6483 & 8 & $\begin{array}{l}129.6666666 \\
666667 \\
\end{array}$ & 184 & 74 & 131 & 36 & 128 & 37 & 5.905e-12 & $1.302 \mathrm{e}-48$ & $3.32 \mathrm{e}-10$ \\
\hline c5 & 727 & 485 & 9 & $\begin{array}{l}131.333333 \\
333333\end{array}$ & 135 & 136 & 123 & 13 & 27 & 11 & 7.391e-15 & $1.739 \mathrm{e}-33$ & $9.485 \mathrm{e}-11$ \\
\hline IKBKG & 8517 & 567 & 9 & 132 & 129 & 133 & 134 & 14 & 29 & 11 & $3.044 \mathrm{e}-15$ & $3.038 \mathrm{e}-34$ & 4.434e- 10 \\
\hline $\begin{array}{l}\text { CTNNB } \\
1 \\
\end{array}$ & 1499 & 5958 & 13 & 134 & 64 & 144 & 194 & 63 & 82 & 28 & $1.118 \mathrm{e}-23$ & 4.129e-32 & \begin{tabular}{|l|}
0.0000001 \\
199 \\
\end{tabular} \\
\hline CEBPA & 1050 & 898 & 9 & $\begin{array}{l}134.3333333 \\
333333 \\
\end{array}$ & 90 & 243 & 70 & 20 & 23 & 19 & 7.217e-19 & $1.601 \mathrm{e}-22$ & $7.18 \mathrm{e}-16$ \\
\hline IL4 & 3565 & 2440 & 7 & $\begin{array}{l}138.3333333 \\
333333 \\
\end{array}$ & 115 & 31 & 269 & 27 & 118 & 13 & $2.734 \mathrm{e}-16$ & $2.004 e-80$ & \begin{tabular}{|l|}
0.0000138 \\
6 \\
\end{tabular} \\
\hline PTEN & 5728 & 3558 & 12 & \begin{tabular}{|l|l|}
139.3333333 \\
333333
\end{tabular} & 80 & 173 & 165 & 41 & 55 & 22 & 9.762e-21 & $3.845 \mathrm{e}-29$ & \begin{tabular}{|l|}
0.0000000 \\
1814 \\
\end{tabular} \\
\hline IL6ST & 3572 & 518 & 10 & 143 & 163 & 91 & 175 & 12 & 36 & 9 & $2.686 \mathrm{e}-13$ & $1.96 \mathrm{e}-44$ & $\begin{array}{l}0.0000000 \\
3432 \\
\end{array}$ \\
\hline $\begin{array}{l}\text { SLC10A } \\
2\end{array}$ & 6555 & 125 & 13 & 144 & 134 & 270 & 28 & 9 & 12 & 19 & $7.195 \mathrm{e}-15$ & $6.821 \mathrm{e}-21$ & $1.377 \mathrm{e}-31$ \\
\hline $\begin{array}{l}U_{1} \text { UGTIA } \\
\end{array}$ & 54658 & 715 & 22 & $\begin{array}{l}148.3333333 \\
333333 \\
\end{array}$ & 81 & 343 & 21 & 20 & 16 & 38 & 1.135e-20 & $1.201 \mathrm{e}-16$ & $6.787 \mathrm{e}-39$ \\
\hline CYPZЕ1 & 1571 & 845 & 7 & $\begin{array}{l}149.3333333 \\
333333 \\
\end{array}$ & 183 & 192 & 73 & 13 & 26 & 18 & $4.978 \mathrm{e}-12$ & $2.711 \mathrm{e}-26$ & 2.693e- 15 \\
\hline CD274 & 29126 & 1011 & 10 & 152 & 117 & 88 & 251 & 18 & 46 & 9 & 5.441e-16 & 7.31e- 45 & \begin{tabular}{|l|}
0.0000059 \\
58 \\
\end{tabular} \\
\hline socs1 & 8651 & 639 & 10 & \begin{tabular}{|l|l|}
153.3333333 \\
333333
\end{tabular} & 160 & 70 & 230 & 13 & 43 & 8 & $1.909 \mathrm{e}-13$ & $8.999 \mathrm{e}-50$ & $\begin{array}{l}0.0000019 \\
39\end{array}$ \\
\hline GPT & 2875 & 189 & 15 & $\begin{array}{l}153.333333 \\
333333\end{array}$ & 13 & 442 & 5 & 28 & 9 & 53 & \begin{tabular}{|l}
$1.773 e-45$ \\
\end{tabular} & $9.366 \mathrm{e}-14$ & $6.163 \mathrm{e}-88$ \\
\hline STAT1 & 6772 & 1574 & 10 & $\begin{array}{l}156.3333333 \\
333333 \\
\end{array}$ & 167 & 81 & 221 & 18 & 57 & 12 & $4.518 \mathrm{e}-13$ & $2.047 \mathrm{e}-46$ & \begin{tabular}{l|}
0.0000010 \\
43 \\
\end{tabular} \\
\hline IFNAR1 & 3454 & 957 & 11 & \begin{tabular}{|l|l|}
157.3333333 \\
333333
\end{tabular} & 161 & 99 & 212 & 15 & 41 & 10 & $2.122 \mathrm{e}-13$ & $1.34 \mathrm{e}-40$ & \begin{tabular}{|l|}
0.0000005 \\
109 \\
\end{tabular} \\
\hline $\begin{array}{l}\text { SLCO1 } \\
\text { B3 }\end{array}$ & 28234 & 174 & 6 & \begin{tabular}{|l|}
158.6666666 \\
666667
\end{tabular} & 152 & 293 & 31 & 9 & 12 & 19 & $1.291 \mathrm{e}-13$ & $3.657 \mathrm{e}-19$ & $9.145 \mathrm{e}-29$ \\
\hline KRT7 & 3855 & 165 & 9 & 159 & 103 & 287 & 87 & 11 & 12 & 10 & $3.313 \mathrm{e}-17$ & 1.954e-19 & $5.957 \mathrm{e}-14$ \\
\hline $\begin{array}{l}\text { ANGPT } \\
2\end{array}$ & 285 & 621 & 10 & $\begin{array}{l}159.666666 \\
666667\end{array}$ & 104 & 147 & 228 & 16 & 28 & 8 & $4.699 \mathrm{e}-17$ & $6.074 \mathrm{e}-32$ & $\begin{array}{l}0.0000015 \\
82 \\
\end{array}$ \\
\hline $\begin{array}{l}\text { TNFFF1 } \\
0\end{array}$ & 8743 & 946 & 10 & \begin{tabular}{|l|l|}
160.3333333 \\
333333
\end{tabular} & 158 & 112 & 211 & 15 & 38 & 10 & $1.845 \mathrm{e}-13$ & $1.229 \mathrm{e}-37$ & \begin{tabular}{|l|}
0.0000004 \\
629 \\
\end{tabular} \\
\hline IGF1 & 3479 & 3110 & 10 & \begin{tabular}{|l|l|}
161.333333 \\
333333
\end{tabular} & 97 & 246 & 141 & 33 & 40 & 23 & 1.115e-17 & $2.104 \mathrm{e}-22$ & $6.685 \mathrm{e}-10$ \\
\hline $\begin{array}{l}\text { TNFAIP } \\
3\end{array}$ & 7128 & 463 & 9 & $\begin{array}{ll}163.666666 \\
666667 \\
\end{array}$ & 174 & 119 & 198 & 11 & 29 & 8 & 1.472e-12 & $1.084 \mathrm{e}-36$ & $\begin{array}{l}0.0000002 \\
01 \\
\end{array}$ \\
\hline FAH & 2184 & 163 & 17 & \begin{tabular}{|l|l|}
164.6666666 \\
666667
\end{tabular} & 123 & 285 & 86 & 10 & 12 & 10 & 1.473e-15 & $1.696 \mathrm{e}-19$ & $5.328 \mathrm{e}-14$ \\
\hline EDN1 & 1906 & 1574 & 10 & \begin{tabular}{|l|}
165.6666666 \\
666667 \\
\end{tabular} & 96 & 256 & 145 & 24 & 28 & 16 & \begin{tabular}{|l}
$6.104 \mathrm{e}-18$ \\
\end{tabular} & $8.575 \mathrm{e}-22$ & $\begin{array}{l}0.0000000 \\
01115 \\
\end{array}$ \\
\hline ILIRN & 3557 & 1296 & 8 & 166 & 173 & 29 & 296 & 16 & 93 & 9 & 1.425e-12 & $9.991 \mathrm{e}-85$ & $\begin{array}{l}0.0000353 \\
3\end{array}$ \\
\hline $\mathrm{C}_{3}$ & 718 & 1180 & 21 & 167 & 131 & 132 & 238 & 18 & 38 & 10 & 6.014e- 15 & $2.36 \mathrm{e}-34$ & $\begin{array}{l}0.0000028 \\
88\end{array}$ \\
\hline FGF21 & 26291 & 429 & 6 & 169 & 168 & 264 & 75 & 11 & 17 & 14 & \begin{tabular}{|l}
$6.786 \mathrm{e}-13$ \\
\end{tabular} & $2.609 \mathrm{e}-21$ & $5.121 \mathrm{e}-15$ \\
\hline ENPP2 & 5168 & 247 & 11 & 169 & 85 & 333 & 89 & 14 & 12 & 11 & 5.236e-20 & 2.2077- 17 & $9.608 \mathrm{e}-14$ \\
\hline ILIR1 & 3554 & 846 & 11 & 170 & 146 & 37 & 327 & 15 & 69 & 7 & $4.269 \mathrm{e}-14$ & $8.857 \mathrm{e}-74$ & \begin{tabular}{|l}
0.0000999 \\
1 \\
\end{tabular} \\
\hline CAV1 & 857 & 1621 & 10 & 170 & 198 & 182 & 130 & 16 & 35 & 17 & $2.815 \mathrm{e}-11$ & $2.794 \mathrm{e}-27$ & $3.005 \mathrm{e}-10$ \\
\hline RBP4 & 5950 & 430 & 9 & $\begin{array}{l}171.6666666 \\
666667 \\
\end{array}$ & 124 & 303 & 88 & 13 & 15 & 13 & $1.773 \mathrm{e}-15$ & $1.326 \mathrm{e}-18$ & $9.266 \mathrm{e}-14$ \\
\hline $\begin{array}{l}\text { NAMP } \\
\end{array}$ & 10135 & 578 & 15 & \begin{tabular}{|l|}
172.6666666 \\
666667
\end{tabular} & 170 & 188 & 160 & 12 & 23 & 10 & 9.036e-13 & $1.432 \mathrm{e}-26$ & $\begin{array}{l}0.00000000 \\
06631 \\
\end{array}$ \\
\hline $\begin{array}{l}\text { PPARG } \\
\text { C1A }\end{array}$ & 10891 & 1286 & 9 & \begin{tabular}{|l|l|}
173.3333333 \\
333333
\end{tabular} & 210 & 200 & 110 & 14 & 30 & 17 & 7.303e-11 & $1.005 \mathrm{e}-25$ & $1.212 \mathrm{e}-11$ \\
\hline IL13 & 3596 & 1096 & 7 & 174 & 101 & 28 & 393 & 20 & 89 & 7 & $2.464 \mathrm{e}-17$ & $6.904 \mathrm{e}-87$ & 0.0004115 \\
\hline TP53 & 7157 & 12916 & 9 & \begin{tabular}{|l|}
175.333333 \\
333333
\end{tabular} & 108 & 148 & 270 & 79 & 140 & 39 & $1.237 \mathrm{e}-16$ & $8.161 \mathrm{e}-32$ & $\begin{array}{l}0.0000145 \\
2\end{array}$ \\
\hline Nos3 & 4846 & 3360 & 8 & 177 & 120 & 224 & 187 & 31 & 44 & 20 & \begin{tabular}{|l}
$9.299 \mathrm{e}-16$ \\
\end{tabular} & $1.011 \mathrm{e}-23$ & $\begin{array}{l}0.00000000 \\
8043 \\
\end{array}$ \\
\hline CNR2 & 1269 & 480 & 11 & 178 & 175 & 237 & 122 & 11 & 19 & 11 & $2.144 \mathrm{e}-12$ & $4.038 \mathrm{e}-23$ & $8.578 \mathrm{e}-11$ \\
\hline
\end{tabular}


bioRxiv preprint doi: https://doi.org/10.1101/2021.09.06.459063; this version posted September 6, 2021. The copyright holder for this preprint (which was not certified by peer review) is the author/funder. All rights reserved. No reuse allowed without permission.

\begin{tabular}{|c|c|c|c|c|c|c|c|c|c|c|c|c|c|}
\hline GOLM1 & 51280 & 115 & 6 & $\begin{array}{l}178.666666 \\
666667 \\
\end{array}$ & 78 & 326 & 132 & 12 & 10 & 7 & $8.372 \mathrm{e}-21$ & $1.585 \mathrm{e}-17$ & $3.532 \mathrm{e}-10$ \\
\hline DDIT3 & 1649 & 532 & 8 & $\begin{array}{l}181.333333 \\
333333 \\
\end{array}$ & 216 & 175 & 153 & 10 & 24 & 10 & $9.492 \mathrm{e}-11$ & $1.231 \mathrm{e}-28$ & $\begin{array}{l}0.0000000 \\
03176\end{array}$ \\
\hline $\mathrm{NR} 1 \mathrm{H} 2$ & 7376 & 933 & 10 & $\begin{array}{l}181.666666 \\
666667 \\
\end{array}$ & 263 & 100 & 182 & 11 & 40 & 11 & $\begin{array}{l}0.00000000 \\
1364\end{array}$ & $5.977 \mathrm{e}-40$ & $\begin{array}{l}0.00000000 \\
509\end{array}$ \\
\hline FABP1 & 2168 & 312 & 9 & $\begin{array}{l}181.666666 \\
666667 \\
\end{array}$ & 144 & 353 & 48 & 11 & 12 & 19 & $2.596 \mathrm{e}-14$ & $3.352 \mathrm{e}-16$ & $4.589 \mathrm{e}-24$ \\
\hline CPS1 & 1373 & 189 & 12 & 183 & 109 & 336 & 104 & 11 & 11 & 9 & $1.417 \mathrm{e}-16$ & $4.597 \mathrm{e}-17$ & $8.482 \mathrm{e}-12$ \\
\hline $\mathrm{CD} 36$ & 948 & 873 & 14 & $\begin{array}{l}184.3333333 \\
333333 \\
\end{array}$ & 211 & 170 & 172 & 12 & 29 & 11 & 7.477e-11 & $3.561 \mathrm{e}-29$ & $\begin{array}{l}0.0000000 \\
2784\end{array}$ \\
\hline KEAP1 & 9817 & 535 & 10 & 185 & 217 & 211 & 127 & 10 & 21 & 11 & $9.962 \mathrm{e}-11$ & $8.808 \mathrm{e}-25$ & $2.559 \mathrm{e}-10$ \\
\hline SLCAA2 & 6522 & 195 & 14 & $\begin{array}{l}186.333333 \\
333333 \\
\end{array}$ & 111 & 339 & 109 & 11 & 11 & 9 & $1.967 \mathrm{e}-16$ & $6.441 \mathrm{e}-17$ & $1.071 \mathrm{e}-11$ \\
\hline AHSG & 197 & 367 & 10 & 188 & 132 & 282 & 150 & 12 & 15 & 9 & $6.065 \mathrm{e}-15$ & $1.396 \mathrm{e}-19$ & $\begin{array}{l}0.0000000 \\
02071\end{array}$ \\
\hline RETN & 56729 & 656 & 5 & \begin{tabular}{|l|l|}
188.3333333 \\
333333
\end{tabular} & 202 & 129 & 234 & 11 & 31 & 8 & $4.881 \mathrm{e}-11$ & $6.844 \mathrm{e}-35$ & $\begin{array}{l}0.0000023 \\
13\end{array}$ \\
\hline RELA & 5970 & 2194 & 10 & 191 & 260 & 64 & 249 & 16 & 75 & 13 & $\begin{array}{l}0.00000000 \\
1227 \\
\end{array}$ & $9.1 \mathrm{e}-53$ & $\begin{array}{l}0.0000051 \\
32\end{array}$ \\
\hline LYZ & 4069 & 1250 & 10 & $\begin{array}{l}191.666666 \\
666667 \\
\end{array}$ & 206 & 22 & 347 & 14 & 101 & 8 & $5.237 \mathrm{e}-11$ & $8.402 \mathrm{e}-94$ & 0.0001554 \\
\hline HP & 3240 & 520 & 7 & $\begin{array}{l}193.3333333 \\
333333 \\
\end{array}$ & 105 & 265 & 210 & 15 & 18 & 8 & $6.026 \mathrm{e}-17$ & $3.131 \mathrm{e}-21$ & $\begin{array}{l}0.0000004 \\
557\end{array}$ \\
\hline SMAD2 & 4087 & 1072 & 10 & $\begin{array}{l}195.333333 \\
333333\end{array}$ & 150 & 209 & 227 & 16 & 27 & 10 & $1.087 \mathrm{e}-13$ & $7.387 \mathrm{e}-25$ & $\begin{array}{l}0.0000013 \\
07\end{array}$ \\
\hline $\begin{array}{l}\text { HLA- } \\
\text { DRB1 }\end{array}$ & 3123 & 2535 & 4 & $\begin{array}{l}195.3333333 \\
333333\end{array}$ & 61 & 401 & 124 & 39 & 24 & 22 & $6.928 \mathrm{e}-24$ & $1.976 \mathrm{e}-14$ & $9.53 \mathrm{e}-11$ \\
\hline $\begin{array}{l}\text { CDKN1 } \\
\text { A }\end{array}$ & 1026 & 2488 & 7 & $\begin{array}{l}200.666666 \\
666667\end{array}$ & 156 & 202 & 244 & 23 & 40 & 14 & $1.726 \mathrm{e}-13$ & $2.35 \mathrm{e}-25$ & $\begin{array}{l}0.0000044 \\
51\end{array}$ \\
\hline TIMP3 & 7078 & 470 & 9 & $\begin{array}{l}200.666666 \\
666667 \\
\end{array}$ & 246 & 212 & 144 & 9 & 20 & 10 & $5.252 \mathrm{e}-10$ & $1.387 \mathrm{e}-24$ & $\begin{array}{l}0.0000000 \\
01042\end{array}$ \\
\hline HIF1A & 3091 & 4151 & 9 & $\begin{array}{l}201.3333333 \\
333333\end{array}$ & 137 & 116 & 351 & 33 & 75 & 15 & $8.425 \mathrm{e}-15$ & $6.417 \mathrm{e}-37$ & 0.0001752 \\
\hline MAPK8 & 5599 & 2419 & 13 & $\begin{array}{l}202.333333 \\
333333 \\
\end{array}$ & 276 & 169 & 162 & 16 & 45 & 18 & $\begin{array}{l}0.00000000 \\
4064\end{array}$ & $3.324 \mathrm{e}-29$ & $\begin{array}{l}0.0000000 \\
1354\end{array}$ \\
\hline IFNL4 & $\begin{array}{r}10118 \\
0976 \\
\end{array}$ & 61 & & $\begin{array}{l}203.333333 \\
333333 \\
\end{array}$ & 71 & 437 & 102 & 11 & 7 & 7 & $5.167 \mathrm{e}-22$ & $7.526 \mathrm{e}-14$ & $4.791 \mathrm{e}-12$ \\
\hline ANXA1 & 301 & 530 & 10 & $\begin{array}{l}209.666666 \\
666667 \\
\end{array}$ & 264 & 152 & 213 & 9 & 26 & 8 & $\begin{array}{l}0.00000000 \\
1375\end{array}$ & $3.245 \mathrm{e}-31$ & $\begin{array}{l}0.0000005 \\
185\end{array}$ \\
\hline $\mathrm{CDH} 1$ & 999 & 3239 & 14 & $\begin{array}{l}209.666666 \\
666667 \\
\end{array}$ & 143 & 210 & 276 & 28 & 45 & 15 & $2.107 \mathrm{e}-14$ & $7.922 \mathrm{e}-25$ & $\begin{array}{l}0.0000174 \\
7\end{array}$ \\
\hline sox9 & 6662 & 1727 & 10 & $\begin{array}{l}210.333333 \\
333333 \\
\end{array}$ & 164 & 278 & 189 & 19 & 27 & 14 & $2.951 \mathrm{e}-13$ & $4.922 \mathrm{e}-20$ & $\begin{array}{l}0.0000000 \\
978\end{array}$ \\
\hline ACTA2 & 59 & 1372 & 12 & 211 & 110 & 262 & 261 & 21 & 26 & 10 & $1.56 \mathrm{e}-16$ & $1.906 \mathrm{e}-21$ & $\begin{array}{l}0.0000096 \\
32\end{array}$ \\
\hline TIMP2 & 7077 & 652 & 10 & 212 & 162 & 308 & 166 & 13 & 17 & 10 & $2.414 \mathrm{e}-13$ & $2.104 \mathrm{e}-18$ & $\begin{array}{l}0.0000000 \\
1968\end{array}$ \\
\hline CD40 & 958 & 1130 & 9 & 213 & 196 & 121 & 322 & 14 & 39 & 8 & $1.56 \mathrm{e}-11$ & $5.816 \mathrm{e}-36$ & $\begin{array}{l}0.0000835 \\
4 \\
\end{array}$ \\
\hline EPCAM & 4072 & 381 & 10 & $\begin{array}{l}213.3333333 \\
333333\end{array}$ & 100 & 273 & 267 & 14 & 16 & 6 & $1.809 \mathrm{e}-17$ & $9.388 \mathrm{e}-21$ & $\begin{array}{l}0.0000131 \\
7\end{array}$ \\
\hline IL12B & 3593 & 1145 & 10 & $\begin{array}{l}215.666666 \\
666667 \\
\end{array}$ & 220 & 194 & 233 & 13 & 29 & 10 & $1.506 \mathrm{e}-10$ & $4.335 \mathrm{e}-26$ & $\begin{array}{l}0.0000022 \\
69 \\
\end{array}$ \\
\hline CXCL9 & 4283 & 160 & 4 & $\begin{array}{l}216.3333333 \\
333333 \\
\end{array}$ & 181 & 316 & 152 & 8 & 11 & 7 & $3.048 \mathrm{e}-12$ & $7.658 \mathrm{e}-18$ & $\begin{array}{l}0.0000000 \\
03128\end{array}$ \\
\hline AHR & 196 & 1062 & 10 & $\begin{array}{l}216.666666 \\
666667 \\
\end{array}$ & 207 & 135 & 308 & 13 & 36 & 8 & $6.643 \mathrm{e}-11$ & $6.529 \mathrm{e}-34$ & $\begin{array}{l}0.0000566 \\
3 \\
\end{array}$ \\
\hline TLR7 & 51284 & 680 & 10 & 220 & 253 & 118 & 289 & 10 & 33 & 7 & $8.251 \mathrm{e}-10$ & $8.578 \mathrm{e}-37$ & $\begin{array}{l}0.0000282 \\
4\end{array}$ \\
\hline $\mathrm{FN} 1$ & 2335 & 1314 & 9 & $\begin{array}{l}220.6666666 \\
666667 \\
\end{array}$ & 87 & 277 & 298 & 24 & 24 & 9 & $1.506 \mathrm{e}-19$ & $4.729 \mathrm{e}-20$ & $\begin{array}{l}0.0000390 \\
1\end{array}$ \\
\hline ATP7B & 540 & 371 & 12 & $\begin{array}{l}221.333333 \\
333333\end{array}$ & 133 & 481 & 50 & 12 & 10 & 19 & $6.836 \mathrm{e}-15$ & $1.321 \mathrm{e}-12$ & $1.144 \mathrm{e}-22$ \\
\hline socs3 & 9021 & 889 & 11 & $\begin{array}{l}222.666666 \\
666667 \\
\end{array}$ & 190 & 46 & 432 & 13 & 62 & 6 & $8.806 \mathrm{e}-12$ & $1.155 \mathrm{e}-64$ & 0.000894 \\
\hline ACE & 1636 & 2786 & 11 & 224 & 130 & 354 & 188 & 27 & 28 & 18 & $4.101 \mathrm{e}-15$ & $3.371 \mathrm{e}-16$ & $\begin{array}{l}0.0000000 \\
8158\end{array}$ \\
\hline AREG & 374 & 305 & 9 & $\begin{array}{l}224.6666666 \\
666667 \\
\end{array}$ & 142 & 290 & 242 & 11 & 14 & 6 & $2.06 \mathrm{e}-14$ & $2.906 \mathrm{e}-19$ & $\begin{array}{l}0.0000040 \\
34\end{array}$ \\
\hline EGR1 & 1958 & 1049 & 10 & $\begin{array}{l}225.333333 \\
333333\end{array}$ & 186 & 266 & 224 & 14 & 23 & 10 & $6.432 \mathrm{e}-12$ & $3.909 \mathrm{e}-21$ & $\begin{array}{l}0.0000010 \\
93\end{array}$ \\
\hline TLR9 & 54106 & 1359 & 9 & 226 & 256 & 51 & 371 & 13 & 67 & 8 & $9.316 \mathrm{e}-10$ & $8.884 \mathrm{e}-59$ & 0.0002566 \\
\hline VDR & 7421 & 2181 & 11 & 226 & 141 & 335 & 202 & 23 & 26 & 15 & $1.653 \mathrm{e}-14$ & $4.369 \mathrm{e}-17$ & $\begin{array}{l}0.0000002 \\
738\end{array}$ \\
\hline $\begin{array}{l}\text { TNFRSF } \\
12 \mathrm{~A}\end{array}$ & 51330 & 164 & 7 & $\begin{array}{l}226.666666 \\
666667\end{array}$ & 89 & 90 & 501 & 12 & 26 & 3 & $5.849 \mathrm{e}-19$ & $1.862 \mathrm{e}-44$ & 0.003109 \\
\hline GFER & 2671 & 136 & 10 & $\begin{array}{l}228.666666 \\
666667\end{array}$ & 204 & 302 & 180 & 7 & 11 & 6 & $5.088 \mathrm{e}-11$ & $1.294 \mathrm{e}-18$ & $\begin{array}{l}0.0000000 \\
4615\end{array}$ \\
\hline AGT & 183 & 1702 & 9 & $\begin{array}{l}230.666666 \\
666667 \\
\end{array}$ & 102 & 358 & 232 & 24 & 22 & 12 & $2.889 \mathrm{e}-17$ & $3.663 \mathrm{e}-16$ & $\begin{array}{l}0.0000021 \\
66\end{array}$ \\
\hline FGFR4 & 2264 & 411 & 10 & $\begin{array}{l}231.666666 \\
666667 \\
\end{array}$ & 225 & 356 & 114 & 9 & 13 & 11 & $1.796 \mathrm{e}-10$ & $3.491 \mathrm{e}-16$ & $1.788 \mathrm{e}-11$ \\
\hline MMP3 & 4314 & 915 & 14 & $\begin{array}{l}232.6666666 \\
666667 \\
\end{array}$ & 151 & 312 & 235 & 15 & 19 & 9 & $1.221 \mathrm{e}-13$ & $3.409 \mathrm{e}-18$ & $\begin{array}{l}0.0000028 \\
61\end{array}$ \\
\hline $\begin{array}{l}\text { NOTCH } \\
1\end{array}$ & 4851 & 4375 & 12 & 233 & 188 & 257 & 254 & 28 & 47 & 19 & $6.88 \mathrm{e}-12$ & $1.132 \mathrm{e}-21$ & $\begin{array}{l}0.0000063 \\
65\end{array}$ \\
\hline
\end{tabular}




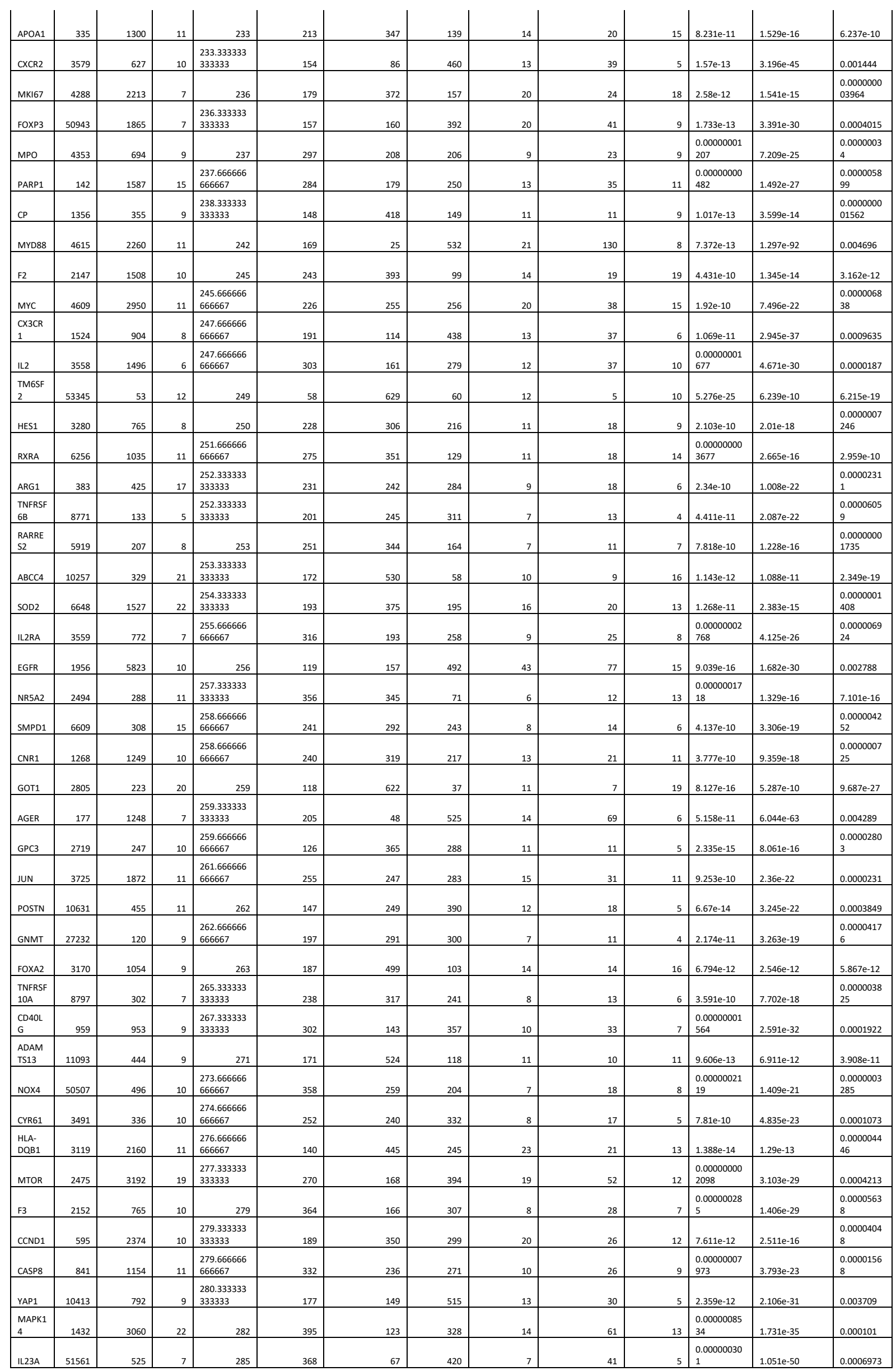




\begin{tabular}{|c|c|c|c|c|c|c|c|c|c|c|c|c|c|}
\hline VCAM1 & 7412 & 879 & 8 & 285 & 390 & 131 & 334 & 8 & 34 & 7 & \begin{tabular}{|l}
0.000000073 \\
94
\end{tabular} & $1.894 \mathrm{e}-34$ & 0.0001243 \\
\hline CD44 & 960 & 1581 & 9 & \begin{tabular}{|l|}
285.3333333 \\
333333 \\
\end{tabular} & 282 & 233 & 341 & 13 & 30 & 9 & \begin{tabular}{|l}
0.000000000 \\
4554 \\
\end{tabular} & $2.026 \mathrm{e}-23$ & 0.0001379 \\
\hline G6PC & 2538 & 208 & 11 & 288 & 88 & 731 & 45 & 13 & 6 & 18 & 2.193e-19 & 0.00000001227 & $9.569 \mathrm{e}-26$ \\
\hline $\begin{array}{l}\text { TGFBR } \\
1\end{array}$ & 7046 & 889 & 14 & \begin{tabular}{|l|l|}
291.333333 \\
333333
\end{tabular} & 136 & 307 & 431 & 16 & 19 & 6 & $7.775 \mathrm{e}-15$ & $2.09 \mathrm{e}-18$ & 0.000896 \\
\hline TERT & 7015 & 1760 & 9 & $\begin{array}{l}294.333333 \\
333333 \\
\end{array}$ & 165 & 479 & 239 & 19 & 18 & 12 & $3.982 \mathrm{e}-13$ & $8.785 \mathrm{e}-13$ & \begin{tabular}{|l|}
0.0000029 \\
25 \\
\end{tabular} \\
\hline FGL2 & 10875 & 99 & 10 & $\begin{array}{l}295.666666 \\
666667 \\
6\end{array}$ & 242 & 217 & 428 & 6 & 13 & 3 & 4.276e-10 & $4.277 \mathrm{e}-24$ & 0.0008178 \\
\hline CFLAR & 8837 & 480 & 10 & 298 & 355 & 238 & 301 & 7 & 19 & 6 & \begin{tabular}{|l|}
0.00000017 \\
17
\end{tabular} & $4.021 \mathrm{e}-23$ & \begin{tabular}{|l|}
0.0000434 \\
4 \\
\end{tabular} \\
\hline DPP4 & 1803 & 606 & 19 & $\begin{array}{l}298.6666666 \\
666667 \\
\end{array}$ & 327 & 377 & 192 & 8 & 14 & 9 & \begin{tabular}{|l|}
0.000000005 \\
561 \\
\end{tabular} & $2.528 \mathrm{e}-15$ & \begin{tabular}{|l}
0.0000001 \\
177 \\
\end{tabular} \\
\hline HSPAS & 3309 & 1303 & 23 & 303 & 279 & 434 & 196 & 12 & 17 & 12 & \begin{tabular}{|l|}
0.000000000 \\
4294
\end{tabular} & $7.402 \mathrm{e}-14$ & $\begin{array}{l}0.0000001 \\
712\end{array}$ \\
\hline CLDN1 & 9076 & 351 & 10 & $\begin{array}{l}303.333333 \\
333333 \\
\end{array}$ & 203 & 369 & 338 & 9 & 12 & 5 & 5.016e-11 & $1.286 \mathrm{e}-15$ & 0.0001299 \\
\hline SPHK1 & 8877 & 465 & 12 & $\begin{array}{l}306.666666 \\
666667\end{array}$ & 349 & 274 & 297 & 7 & 17 & 6 & $\begin{array}{l}0.00000014 \\
12\end{array}$ & $9.532 \mathrm{e}-21$ & 0.0000368 \\
\hline CBSL & $\begin{array}{r}10272 \\
4560 \\
\end{array}$ & 246 & 22 & $\begin{array}{l}08.3333333 \\
333333 \\
\end{array}$ & 417 & 411 & 97 & 5 & 10 & 10 & \begin{tabular}{|l|}
0.00000211 \\
5
\end{tabular} & $2.723 \mathrm{e}-14$ & $2.876 \mathrm{e}-12$ \\
\hline TXNIP & 10628 & 319 & 10 & 314 & 369 & 325 & 248 & 6 & 13 & 6 & \begin{tabular}{|l|}
0.00000030 \\
14 \\
\end{tabular} & $1.518 \mathrm{e}-17$ & \begin{tabular}{|l|}
0.0000051 \\
05 \\
\end{tabular} \\
\hline CST3 & 1471 & 605 & 9 & \begin{tabular}{|l|l|}
316.3333333 \\
333333 \\
\end{tabular} & 149 & 658 & 142 & 13 & 9 & 11 & $1.045 \mathrm{e}-13$ & 0.000000001708 & $8.181 \mathrm{e}-10$ \\
\hline IL21 & 59067 & 520 & 7 & 317 & 259 & 134 & 558 & 9 & 28 & 4 & \begin{tabular}{|l|}
0.00000000 \\
1191 \\
\end{tabular} & $5.4011-34$ & 0.006427 \\
\hline STAT6 & 6778 & 869 & 9 & $\begin{array}{l}317.666666 \\
666667 \\
\end{array}$ & 454 & 72 & 427 & 7 & 47 & 6 & 0.00000655 & $1.018 \mathrm{e}-48$ & 0.0008009 \\
\hline CXCL2 & 2920 & 370 & 8 & $\begin{array}{l}317.6666666 \\
666667\end{array}$ & 387 & 85 & 481 & 6 & 33 & 4 & \begin{tabular}{|l}
0.00000068 \\
09
\end{tabular} & $3.112 \mathrm{e}-45$ & 0.002085 \\
\hline STAT4 & 6775 & 533 & 10 & $\begin{array}{l}318.6666666 \\
666667 \\
\end{array}$ & 265 & 268 & 423 & 9 & 18 & 5 & \begin{tabular}{|l|}
0.000000000 \\
1438
\end{tabular} & $4.752 \mathrm{e}-21$ & 0.0007428 \\
\hline PDGFB & 5155 & 683 & 10 & $\begin{array}{l}319.3333333 \\
333333 \\
\end{array}$ & 166 & 313 & 479 & 13 & 17 & 5 & $4.16 \mathrm{e}-13$ & $4.37 \mathrm{e}-18$ & 0.002047 \\
\hline PPARD & 5467 & 694 & 11 & \begin{tabular}{|l|}
320.666666 \\
666667 \\
\end{tabular} & 412 & 176 & 374 & 7 & 26 & 6 & 0.00000169 & $2.373 \mathrm{e}-28$ & 0.0002713 \\
\hline $\begin{array}{l}\text { HAVCR } \\
2\end{array}$ & 84868 & 340 & 6 & \begin{tabular}{|l|}
321.6666666 \\
666667
\end{tabular} & 306 & 196 & 463 & 7 & 19 & 4 & \begin{tabular}{|l}
0.000000001 \\
946
\end{tabular} & $7.837 \mathrm{e}-26$ & 0.001571 \\
\hline LIPA & 3988 & 129 & 23 & $\begin{array}{l}223.333333 \\
333333 \\
\end{array}$ & 340 & 537 & 93 & 5 & 7 & 9 & $\begin{array}{l}0.00000010 \\
6\end{array}$ & $1.331 \mathrm{e}-11$ & $2.981 \mathrm{e}-13$ \\
\hline ІКвКВ & 3551 & 894 & 10 & 324 & 463 & 75 & 434 & 7 & 47 & 6 & \begin{tabular}{|l|}
$\begin{array}{l}0.00000769 \\
9\end{array}$ \\
\end{tabular} & $3.443 \mathrm{e}-48$ & 0.0009164 \\
\hline BID & 637 & 449 & 8 & $\begin{array}{l}324.666666 \\
666667\end{array}$ & 345 & 337 & 292 & 7 & 14 & 6 & \begin{tabular}{|l|} 
\\
3 \\
3
\end{tabular} & 5.034e-17 & \begin{tabular}{|l|}
0.0000307 \\
1
\end{tabular} \\
\hline IDO1 & 3620 & 551 & 11 & 326 & 566 & 197 & 215 & 5 & 22 & 8 & 0.00007577 & $9.02 \mathrm{e}-26$ & $\begin{array}{l}0.0000006 \\
876\end{array}$ \\
\hline TGFB2 & 7042 & 738 & 10 & $\begin{array}{l}328.3333333 \\
333333 \\
\end{array}$ & 221 & 462 & 302 & 11 & 13 & 7 & $1.501 \mathrm{e}-10$ & $3.842 \mathrm{e}-13$ & $\begin{array}{l}0.0000456 \\
1\end{array}$ \\
\hline $\begin{array}{ll}\text { SLC4OA } \\
1\end{array}$ & 30061 & 296 & 13 & $\begin{array}{l}328.6666666 \\
666667 \\
\end{array}$ & 235 & 511 & 240 & 8 & 9 & 6 & 3.108e-10 & $4.445 \mathrm{e}-12$ & \begin{tabular}{|l|}
0.0000034 \\
19 \\
\end{tabular} \\
\hline STK11 & 6794 & 659 & 11 & $\begin{array}{l}329.333333 \\
333333 \\
\end{array}$ & 182 & 443 & 363 & 12 & 13 & 6 & \begin{tabular}{|l|}
$3.743 \mathrm{e}-12$ \\
\end{tabular} & $1.006 \mathrm{e}-13$ & 0.0002105 \\
\hline $\begin{array}{l}\text { PROM } \\
1 \\
\end{array}$ & 8842 & 609 & 11 & $\begin{array}{l}329.6666666 \\
666667 \\
6\end{array}$ & 236 & 298 & 455 & 10 & 17 & 5 & 3.134e-10 & $7.222 \mathrm{e}-19$ & 0.001277 \\
\hline СхзС૮1 & 6376 & 436 & 7 & \begin{tabular}{|l|}
331.3333333 \\
333333 \\
\end{tabular} & 337 & 146 & 511 & 7 & 25 & 4 & \begin{tabular}{|l|}
0.000000009 \\
498 \\
\end{tabular} & $5.715 e-32$ & 0.003642 \\
\hline KLF6 & 1316 & 224 & 10 & \begin{tabular}{|l|}
331.3333333 \\
333333
\end{tabular} & 145 & 286 & 563 & 10 & 13 & 3 & $3.04 \mathrm{e}-14$ & $1.779 \mathrm{e}-19$ & 0.00687 \\
\hline ITGAV & 3685 & 824 & 10 & 332 & 281 & 391 & 324 & 10 & 15 & 7 & \begin{tabular}{|l|}
0.000000000 \\
4434 \\
\end{tabular} & $1.105 \mathrm{e}-14$ & $\begin{array}{l}0.0000854 \\
4 \\
\end{array}$ \\
\hline$S C D$ & 6319 & 427 & 11 & $\begin{array}{l}332.3333333 \\
333333\end{array}$ & 408 & 454 & 135 & 6 & 11 & 10 & \begin{tabular}{|l|}
0.00000147 \\
7
\end{tabular} & $2.389 \mathrm{e}-13$ & $4.443 \mathrm{e}-10$ \\
\hline ENG & 2022 & 592 & 8 & 333 & 321 & 342 & 336 & 8 & 15 & 6 & $\begin{array}{l}0.00000004 \\
749\end{array}$ & $1.19 \mathrm{e}-16$ & 0.0001258 \\
\hline ANXA2 & 302 & 617 & 11 & 335 & 237 & 422 & 346 & 10 & 13 & 6 & 3.53e-10 & 4.701e-14 & 0.0001536 \\
\hline AGTR1 & 185 & 1558 & 9 & $\begin{array}{l}335.666666 \\
666667 \\
6\end{array}$ & 114 & 404 & 489 & 22 & 19 & 7 & $2.159 \mathrm{e}-16$ & $2.238 \mathrm{e}-14$ & 0.00264 \\
\hline$F 2 R$ & 2149 & 589 & 10 & 336 & 230 & 191 & 587 & 10 & 23 & 4 & $2.344 \mathrm{e}-10$ & $2.139 \mathrm{e}-26$ & 0.009659 \\
\hline FGF7 & 2252 & 342 & 10 & \begin{tabular}{|l|}
336.3333333 \\
333333
\end{tabular} & 308 & 368 & 333 & 7 & 12 & 5 & \begin{tabular}{|l|}
0.00000002 \\
012
\end{tabular} & $9.5 e-16$ & 0.0001164 \\
\hline PLAUR & 5329 & 694 & 6 & 337 & 298 & 340 & 373 & 9 & 16 & 6 & \begin{tabular}{|l|}
0.00000001 \\
203 \\
\end{tabular} & $7.734 \mathrm{e}-17$ & 0.0002721 \\
\hline GSK3B & 2932 & 2095 & 12 & \begin{tabular}{|l|}
338.6666666 \\
666667 \\
\end{tabular} & 333 & 254 & 429 & 13 & 32 & 9 & \begin{tabular}{|l|}
0.000000008 \\
466 \\
\end{tabular} & $7.505 \mathrm{e}-22$ & 0.0008373 \\
\hline GGT1 & 2678 & 314 & 27 & \begin{tabular}{|l|}
338.666666 \\
666667
\end{tabular} & 122 & 827 & 67 & 12 & 6 & 14 & $1.046 \mathrm{e}-15$ & 0.0000001226 & $8.074 \mathrm{e}-17$ \\
\hline AQP1 & 358 & 663 & 14 & \begin{tabular}{|l|}
340.3333333 \\
333333
\end{tabular} & 249 & 486 & 286 & 10 & 12 & 7 & 6.612e-10 & $1.501 \mathrm{e}-12$ & \begin{tabular}{|l|}
0.0000242 \\
5 \\
\end{tabular} \\
\hline SELP & 6403 & 743 & 9 & \begin{tabular}{|l|}
341.3333333 \\
333333
\end{tabular} & 309 & 327 & 388 & 9 & 17 & 6 & \begin{tabular}{|l|l}
0.00000002 \\
056
\end{tabular} & $1.609 \mathrm{e}-17$ & 0.0003793 \\
\hline VWF & 7450 & 1091 & 10 & $\begin{array}{l}43.3333333 \\
333333 \\
\end{array}$ & 250 & 466 & 314 & 12 & 15 & 8 & 7.195e-10 & 4.37e-13 & $\begin{array}{l}0.0000670 \\
4 \\
\end{array}$ \\
\hline ABCB1 & 5243 & 2497 & 23 & $\begin{array}{l}343.333333 \\
333333\end{array}$ & 342 & 521 & 167 & 14 & 20 & 18 & \begin{tabular}{|l|l|}
0.000000010 \\
97
\end{tabular} & $6.188 \mathrm{e}-12$ & $\begin{array}{l}0.0000000 \\
2045 \\
\end{array}$ \\
\hline
\end{tabular}




\begin{tabular}{|c|c|c|c|c|c|c|c|c|c|c|c|c|c|}
\hline CTLA4 & 1493 & 1356 & 9 & \begin{tabular}{|l|}
344.6666666 \\
666667
\end{tabular} & 180 & 400 & 454 & 16 & 18 & 7 & $2.594 \mathrm{e}-12$ & $1.819 \mathrm{e}-14$ & 0.001271 \\
\hline CASP1 & 834 & 931 & 13 & 345 & 400 & 71 & 564 & 8 & 49 & 5 & \begin{tabular}{|l|}
0.00000109 \\
7
\end{tabular} & 1.499e-49 & 0.006924 \\
\hline TREM1 & 54210 & 211 & 7 & 345 & 398 & 87 & 550 & 5 & 28 & 3 & \begin{tabular}{|l|}
0.00000104 \\
3
\end{tabular} & $6.834 \mathrm{e}-45$ & 0.005912 \\
\hline $\begin{array}{l}\text { ENTPD } \\
1\end{array}$ & 953 & 268 & 14 & 345 & 278 & 338 & 419 & 7 & 12 & 4 & \begin{tabular}{|l|}
.000000000 \\
4206
\end{tabular} & $5.76 \mathrm{e}-17$ & 0.0006971 \\
\hline MAPK3 & 5595 & 2900 & 18 & $\begin{array}{l}345.3333333 \\
333333 \\
\end{array}$ & 271 & 219 & 546 & 18 & 41 & 9 & \begin{tabular}{|l|}
0.00000000 \\
2231 \\
\end{tabular} & $6.258 \mathrm{e}-24$ & 0.005833 \\
\hline МТTP & 4547 & 313 & 12 & $\begin{array}{l}347.3333333 \\
333333 \\
\end{array}$ & 363 & 594 & 85 & 6 & 8 & 12 & \begin{tabular}{|l|}
0.00000027 \\
42 \\
\end{tabular} & $1.904 \mathrm{e}-10$ & $4.79 \mathrm{e}-14$ \\
\hline CAB & 721 & 377 & 12 & 348 & 214 & 564 & 266 & 9 & 9 & 6 & 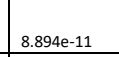 & $3.375 \mathrm{e}-11$ & $\begin{array}{l}0.0000124 \\
5\end{array}$ \\
\hline REG3A & 5068 & 113 & 5 & $\begin{array}{l}350.333333 \\
333333 \\
\end{array}$ & 430 & 171 & 450 & 4 & 16 & 3 & $\begin{array}{l}0.00000333 \\
4\end{array}$ & $3.78 \mathrm{e}-29$ & 0.001153 \\
\hline $\begin{array}{l}\text { TNFSF } 1 \\
3 B\end{array}$ & 10673 & 526 & 10 & $\begin{array}{l}350.666666 \\
666667\end{array}$ & 262 & 228 & 562 & 9 & 20 & 4 & \begin{tabular}{|l|}
0.000000000 \\
1299
\end{tabular} & $1.145 \mathrm{e}-23$ & 0.006656 \\
\hline $\begin{array}{l}\text { SERPIN } \\
\text { A12 }\end{array}$ & $\begin{array}{r}14526 \\
4 \\
\end{array}$ & 125 & 8 & \begin{tabular}{|l|}
351.6666666 \\
666667
\end{tabular} & 199 & 630 & 226 & 7 & 6 & 5 & $2.874 \mathrm{e}-11$ & $6.754 \mathrm{e}-10$ & $\begin{array}{l}0.0000012 \\
46 \\
\end{array}$ \\
\hline THBD & 7056 & 419 & 10 & 353 & 227 & 330 & 502 & 9 & 14 & 4 & $2.105 \mathrm{e}-10$ & $2.009 \mathrm{e}-17$ & 0.003193 \\
\hline CALCA & 796 & 390 & 6 & \begin{tabular}{|l|}
354.3333333 \\
333333 \\
\end{tabular} & 320 & 383 & 360 & 7 & 12 & 5 & $\begin{array}{l}0.00000004 \\
717\end{array}$ & $4.259 \mathrm{e}-15$ & 0.0002013 \\
\hline DLK1 & 8788 & 356 & 9 & 355 & 178 & 547 & 340 & 10 & 9 & 5 & $2.388 \mathrm{e}-12$ & $2.107 \mathrm{e}-11$ & 0.0001382 \\
\hline JAK2 & 3717 & 1649 & 10 & 356 & 287 & 427 & 354 & 13 & 19 & 9 & $\begin{array}{l}0.000000000 \\
7301\end{array}$ & $5.356 \mathrm{e}-14$ & 0.0001814 \\
\hline FGA & 2243 & 465 & 9 & 357 & 421 & 413 & 237 & 6 & 12 & 7 & \begin{tabular}{|l|l|}
0.00000233 \\
6
\end{tabular} & $3.054 \mathrm{e}-14$ & $\begin{array}{l}0.0000028 \\
85 \\
\end{array}$ \\
\hline VIM & 7431 & 1063 & 9 & $\begin{array}{l}357.333333 \\
333333 \\
\end{array}$ & 208 & 555 & 309 & 13 & 13 & 8 & $6.685 \mathrm{e}-11$ & $2.505 \mathrm{e}-11$ & $\begin{array}{l}0.0000568 \\
2\end{array}$ \\
\hline PRF1 & 5551 & 670 & 20 & 358 & 291 & 311 & 472 & 9 & 17 & 5 & \begin{tabular}{|l}
0.000000000 \\
9157 \\
\end{tabular} & $3.23 \mathrm{e}-18$ & 0.001904 \\
\hline CxC112 & 6387 & 1800 & 9 & $\begin{array}{l}358.666666 \\
666667\end{array}$ & 305 & 231 & 540 & 13 & 32 & 7 & \begin{tabular}{|l|}
0.000000001 \\
801
\end{tabular} & $1.394 \mathrm{e}-23$ & 0.005398 \\
\hline CLDN7 & 1366 & 164 & 9 & $\begin{array}{l}361.666666 \\
666667 \\
\end{array}$ & 224 & 361 & 500 & 7 & 10 & 3 & 1.728e-10 & $5.339 \mathrm{e}-16$ & 0.003115 \\
\hline$\angle B P$ & 3929 & 191 & 6 & 363 & 730 & 252 & 107 & 3 & 14 & 9 & 0.0006853 & 4.681e-22 & $9.06 \mathrm{e}-12$ \\
\hline CBS & 875 & 556 & 22 & 363 & 450 & 506 & 133 & 6 & 11 & 11 & $\begin{array}{l}0.00000605 \\
2\end{array}$ & $3.482 \mathrm{e}-12$ & $3.647 \mathrm{e}-10$ \\
\hline $\begin{array}{l}\text { SLCO1 } \\
\text { B1 }\end{array}$ & 10599 & 283 & 5 & 364 & 352 & 671 & 69 & 6 & 7 & 13 & $\begin{array}{l}0.00000015 \\
68\end{array}$ & 0.0000000002533 & $5.835 \mathrm{e}-16$ \\
\hline CXCL3 & 2921 & 256 & 6 & $\begin{array}{l}364.333333 \\
333333 \\
\end{array}$ & 425 & 78 & 590 & 5 & 31 & 3 & $\begin{array}{l}0.00000252 \\
3\end{array}$ & $2.558 \mathrm{e}-47$ & 0.009643 \\
\hline MMP1 & 4312 & 977 & 9 & 365 & 410 & 390 & 295 & 8 & 16 & & \begin{tabular}{|l|}
0.00000151 \\
8
\end{tabular} & $1.084 \mathrm{e}-14$ & $\begin{array}{l}0.0000328 \\
5\end{array}$ \\
\hline sox17 & 64321 & 232 & 9 & 365 & 268 & 546 & 281 & 7 & 8 & 5 & \begin{tabular}{|l|}
0.00000000 \\
1614
\end{tabular} & $1.961 \mathrm{e}-11$ & $\begin{array}{l}0.0000211 \\
8 \\
\end{array}$ \\
\hline ESR1 & 2099 & 4370 & 10 & $\begin{array}{l}365.6666666 \\
666667 \\
\end{array}$ & 247 & 478 & 372 & 24 & 29 & 15 & 5.259e-10 & $8.288 \mathrm{e}-13$ & 0.0002726 \\
\hline CxCL16 & 58191 & 195 & 7 & 366 & 389 & 174 & 535 & 5 & 18 & 3 & $\begin{array}{l}0.00000072 \\
27\end{array}$ & $1.115 \mathrm{e}-28$ & 0.004831 \\
\hline SLC51A & $\begin{array}{r}20093 \\
1 \\
\end{array}$ & 48 & 9 & $\begin{array}{l}666.3333333 \\
333333 \\
\end{array}$ & 254 & 788 & 57 & 5 & 4 & 10 & $9.251 \mathrm{e}-10$ & 0.00000005671 & $2.208 \mathrm{e}-19$ \\
\hline NPC1 & 4864 & 458 & 19 & $\begin{array}{l}367.333333 \\
333333\end{array}$ & 418 & 593 & 91 & 6 & 9 & 13 & \begin{tabular}{|l|l|}
0.00000215 \\
7
\end{tabular} & $1.743 \mathrm{e}-10$ & $1.973 \mathrm{e}-13$ \\
\hline S100A4 & 6275 & 438 & 10 & $\begin{array}{l}367.666666 \\
666667\end{array}$ & 286 & 304 & 513 & 8 & 15 & 4 & \begin{tabular}{|l|}
0.00000000 \\
5361
\end{tabular} & $1.729 \mathrm{e}-18$ & 0.00369 \\
\hline $\begin{array}{l}\text { PDGFR } \\
\text { B }\end{array}$ & 5159 & 908 & 10 & $\begin{array}{l}367.666666 \\
666667 \\
\end{array}$ & 192 & 357 & 554 & 13 & 17 & 5 & $1.122 \mathrm{e}-11$ & $3.507 \mathrm{e}-16$ & 0.006318 \\
\hline HHEX & 3087 & 453 & 10 & $\begin{array}{l}368.333333 \\
333333 \\
\end{array}$ & 346 & 465 & 294 & 7 & 11 & 6 & \begin{tabular}{|l|}
$\begin{array}{l}0.00000011 \\
95\end{array}$ \\
\end{tabular} & $4.371 \mathrm{e}-13$ & $\begin{array}{l}0.0000320 \\
7\end{array}$ \\
\hline MCL1 & 4170 & 782 & 10 & $\begin{array}{l}368.333333 \\
333333 \\
\end{array}$ & 317 & 476 & 312 & 9 & 13 & 7 & \begin{tabular}{|l|}
0.00000003 \\
072
\end{tabular} & $7.521 \mathrm{e}-13$ & $\begin{array}{l}0.0000637 \\
7\end{array}$ \\
\hline CPT1A & 1374 & 239 & 11 & $\begin{array}{l}370.333333 \\
333333 \\
\end{array}$ & 415 & 474 & 222 & 5 & 9 & 6 & \begin{tabular}{l|}
0.00000184 \\
5
\end{tabular} & $7.169 \mathrm{e}-13$ & $\begin{array}{l}0.0000010 \\
61\end{array}$ \\
\hline $\begin{array}{l}\text { CYP3A } \\
4 \\
\end{array}$ & 1576 & 865 & 28 & $\begin{array}{l}370.333333 \\
333333 \\
\end{array}$ & 385 & 556 & 170 & 8 & 12 & 11 & \begin{tabular}{|l|}
0.00000066 \\
62 \\
\end{tabular} & $2.563 \mathrm{e}-11$ & \begin{tabular}{|l|}
0.0000000 \\
257 \\
\end{tabular} \\
\hline $\begin{array}{l}\text { PDCD1 } \\
\mathrm{LG2}\end{array}$ & 80380 & 166 & 8 & 371 & 290 & 320 & 503 & 6 & 11 & 3 & \begin{tabular}{|l|l|}
0.000000000 \\
8068
\end{tabular} & $1.141 \mathrm{e}-17$ & 0.003208 \\
\hline PKD1 & 5310 & 481 & 8 & $\begin{array}{l}371.6666666 \\
666667 \\
\end{array}$ & 176 & 540 & 399 & 11 & 10 & 5 & $2.179 \mathrm{e}-12$ & $1.448 \mathrm{e}-11$ & 0.0004881 \\
\hline ABCG8 & 64241 & 197 & 15 & 372 & 517 & 520 & 79 & 4 & 8 & 11 & 0.00002519 & $5.613 \mathrm{e}-12$ & $9.074 \mathrm{e}-15$ \\
\hline $\begin{array}{l}\text { ANGPT } \\
1\end{array}$ & 284 & 565 & 10 & $\begin{array}{l}372.6666666 \\
666667 \\
\end{array}$ & 223 & 459 & 436 & 10 & 12 & 5 & $1.63 \mathrm{e}-10$ & $2.581 \mathrm{e}-13$ & 0.0009441 \\
\hline GSTP1 & 2950 & 1716 & 15 & $\begin{array}{l}374.666666 \\
666667\end{array}$ & 269 & 549 & 306 & 14 & 16 & 10 & \begin{tabular}{|l|}
.000000000 \\
1884
\end{tabular} & $2.209 \mathrm{e}-11$ & $\begin{array}{l}0.0000530 \\
7\end{array}$ \\
\hline $\begin{array}{l}\text { ADIPO } \\
\text { R1 }\end{array}$ & 51094 & 380 & 20 & $\begin{array}{l}375.333333 \\
333333 \\
\end{array}$ & 392 & 381 & 353 & 6 & 12 & 5 & $\begin{array}{l}0.00000078 \\
46\end{array}$ & $3.16 \mathrm{e}-15$ & 0.0001815 \\
\hline $\begin{array}{l}\text { ADIPO } \\
\text { R2 }\end{array}$ & 79602 & 254 & 13 & 377 & 423 & 299 & 409 & 5 & 13 & 4 & $\begin{array}{l}0.00000244 \\
\end{array}$ & $8.735 \mathrm{e}-19$ & 0.0005807 \\
\hline $\mathrm{BCL2}$ & 596 & 2746 & 8 & $\begin{array}{l}377.3333333 \\
333333 \\
\end{array}$ & 234 & 440 & 458 & 19 & 24 & 10 & $2.731 \mathrm{e}-10$ & $8.159 \mathrm{e}-14$ & 0.001356 \\
\hline $\mathrm{CFH}$ & 3075 & 865 & 27 & $\begin{array}{l}77.3333333 \\
333333 \\
\end{array}$ & 331 & 602 & 199 & 9 & 11 & 10 & \begin{tabular}{|l|}
0.00000006 \\
791
\end{tabular} & $2.779 \mathrm{e}-10$ & \begin{tabular}{|l|}
0.0000002 \\
194 \\
\end{tabular} \\
\hline СЕВРв & 1051 & 904 & 8 & \begin{tabular}{|l|}
377.666666 \\
666667
\end{tabular} & 464 & 232 & 437 & 7 & 24 & 6 & \begin{tabular}{|l|}
0.00000824 \\
7
\end{tabular} & $1.629 \mathrm{e}-23$ & 0.0009657 \\
\hline
\end{tabular}


bioRxiv preprint doi: https://doi.org/10.1101/2021.09.06.459063; this version posted September 6, 2021. The copyright holder for this preprint (which was not certified by peer review) is the author/funder. All rights reserved. No reuse allowed without permission.

\begin{tabular}{|c|c|c|c|c|c|c|c|c|c|c|c|c|c|}
\hline $\begin{array}{l}\text { SULT2 } \\
\text { A1 }\end{array}$ & 6822 & 120 & 14 & $\begin{array}{l}379.333333 \\
333333\end{array}$ & 261 & 771 & 106 & 6 & 5 & 8 & $\begin{array}{l}0.00000000 \\
1274\end{array}$ & 0.00000003253 & $9.129 \mathrm{e}-12$ \\
\hline ITGB6 & 3694 & 177 & 10 & 380 & 295 & 497 & 348 & 6 & 8 & 4 & \begin{tabular}{|l}
0.00000001 \\
164 \\
\end{tabular} & $2.496 \mathrm{e}-12$ & 0.0001671 \\
\hline ALOX5 & 240 & 541 & 16 & $\begin{array}{l}383.333333 \\
333333\end{array}$ & 444 & 280 & 426 & 6 & 17 & 5 & $\begin{array}{l}0.00000525 \\
2\end{array}$ & $1.12 \mathrm{e}-19$ & 0.0007904 \\
\hline TJP1 & 7082 & 779 & 11 & $\begin{array}{l}385.6666666 \\
666667\end{array}$ & 371 & 527 & 259 & 8 & 12 & 8 & $\begin{array}{l}0.00000031 \\
98\end{array}$ & $8.457 \mathrm{e}-12$ & $\begin{array}{l}0.0000073 \\
66\end{array}$ \\
\hline $\begin{array}{l}\text { TNFAIP } \\
8 \mathrm{~L} 2 \\
\end{array}$ & 79626 & 74 & 9 & $\begin{array}{l}386.333333 \\
333333 \\
\end{array}$ & 546 & 227 & 386 & 3 & 12 & 3 & 0.00005425 & $1.079 \mathrm{e}-23$ & 0.0003797 \\
\hline NPPB & 4879 & 926 & 5 & $\begin{array}{l}386.666666 \\
666667 \\
\end{array}$ & 195 & 518 & 447 & 13 & 13 & 6 & $1.396 \mathrm{e}-11$ & $5.254 \mathrm{e}-12$ & 0.001072 \\
\hline CASP3 & 836 & 3210 & 10 & 387 & 497 & 360 & 304 & 12 & 30 & 14 & 0.00001658 & $4.309 \mathrm{e}-16$ & $\begin{array}{l}0.0000497 \\
5\end{array}$ \\
\hline $\begin{array}{l}\text { CYP7B } \\
1 \\
\end{array}$ & 9420 & 108 & 10 & $\begin{array}{l}388.333333 \\
333333\end{array}$ & 319 & 746 & 100 & 5 & 5 & 8 & $\begin{array}{l}0.00000004 \\
63\end{array}$ & 0.0000000198 & $4.106 \mathrm{e}-12$ \\
\hline HNF1A & 6927 & 640 & 10 & $\begin{array}{l}389.666666 \\
666667\end{array}$ & 482 & 539 & 148 & 6 & 11 & 11 & 0.00001249 & $1.416 \mathrm{e}-11$ & $\begin{array}{l}0.0000000 \\
014\end{array}$ \\
\hline RORC & 6097 & 363 & 8 & $\begin{array}{l}391.333333 \\
333333\end{array}$ & 480 & 220 & 474 & 5 & 18 & 4 & 0.00001219 & $6.82 \mathrm{e}-24$ & 0.001968 \\
\hline EGF & 1950 & 1046 & 10 & $\begin{array}{l}391.333333 \\
333333\end{array}$ & 244 & 457 & 473 & 12 & 15 & 6 & $4.675 \mathrm{e}-10$ & $2.518 \mathrm{e}-13$ & 0.001922 \\
\hline PLAU & 5328 & 906 & 10 & $\begin{array}{l}393.333333 \\
333333\end{array}$ & 293 & 334 & 553 & 10 & 18 & 5 & $\begin{array}{l}0.00000001 \\
016\end{array}$ & $3.12 \mathrm{e}-17$ & 0.006265 \\
\hline $\mathrm{NQO} 1$ & 1728 & 743 & 10 & 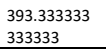 & 222 & 464 & 494 & 11 & 13 & 5 & $1.602 \mathrm{e}-10$ & $4.152 \mathrm{e}-13$ & 0.002905 \\
\hline NR3C2 & 4306 & 465 & 11 & $\begin{array}{l}395.666666 \\
666667 \\
\end{array}$ & 245 & 414 & 528 & 9 & 12 & 4 & $4.808 \mathrm{e}-10$ & $3.047 \mathrm{e}-14$ & 0.004483 \\
\hline IRF1 & 3659 & 572 & 10 & 396 & 458 & 288 & 442 & 6 & 17 & 5 & 0.00000698 & $2.702 \mathrm{e}-19$ & 0.0009857 \\
\hline $\begin{array}{l}\text { HSD11 } \\
\text { B1 }\end{array}$ & 3290 & 437 & 10 & $\begin{array}{l}405.333333 \\
333333\end{array}$ & 411 & 667 & 138 & 6 & 8 & 10 & $\begin{array}{l}0.00000167 \\
6\end{array}$ & 0.000000002259 & $5.425 \mathrm{e}-10$ \\
\hline FGF2 & 2247 & 1553 & 10 & 406 & 350 & 380 & 488 & 11 & 20 & 7 & $\begin{array}{l}0.00000014 \\
78\end{array}$ & $3.151 \mathrm{e}-15$ & 0.002597 \\
\hline $\begin{array}{l}\text { COMM } \\
\text { D1 }\end{array}$ & $\begin{array}{r}15068 \\
4 \\
\end{array}$ & 110 & 10 & $\begin{array}{l}406.666666 \\
666667 \\
\end{array}$ & 324 & 605 & 291 & 5 & 6 & 4 & $\begin{array}{l}0.00000004 \\
999\end{array}$ & $3.243 \mathrm{e}-10$ & $\begin{array}{l}0.0000304 \\
5 \\
\end{array}$ \\
\hline FLT1 & 2321 & 1066 & 8 & $\begin{array}{l}407.666666 \\
666667 \\
\end{array}$ & 283 & 557 & 383 & 11 & 13 & 7 & $\begin{array}{l}0.00000000 \\
4789\end{array}$ & $2.58 \mathrm{e}-11$ & 0.0003566 \\
\hline MST1 & 4485 & 178 & 10 & $\begin{array}{l}408.333333 \\
333333 \\
\end{array}$ & 376 & 500 & 349 & 5 & 8 & 4 & \begin{tabular}{l|l}
0.00000047 \\
62 \\
\end{tabular} & $2.595 \mathrm{e}-12$ & 0.0001704 \\
\hline FOXO3 & 2309 & 947 & 10 & 411 & 301 & 363 & 569 & 10 & 17 & 5 & $\begin{array}{l}0.00000001 \\
481\end{array}$ & $6.651 \mathrm{e}-16$ & 0.007392 \\
\hline APLN & 8862 & 383 & 5 & $\begin{array}{l}412.333333 \\
333333\end{array}$ & 394 & 487 & 356 & 6 & 10 & 5 & \begin{tabular}{|l|}
0.00000081 \\
68 \\
\end{tabular} & $1.778 \mathrm{e}-12$ & 0.0001868 \\
\hline $\begin{array}{l}\text { SLC27A } \\
5 \\
\end{array}$ & 10998 & 38 & 6 & $\begin{array}{l}412.333333 \\
333333 \\
\end{array}$ & 325 & 754 & 158 & 4 & 4 & 5 & \begin{tabular}{|l|}
0.00000005 \\
241 \\
\end{tabular} & 0.00000002259 & $\begin{array}{l}0.0000000 \\
04069 \\
\end{array}$ \\
\hline DGAT2 & 84649 & 110 & 10 & $\begin{array}{l}412.666666 \\
666667 \\
\end{array}$ & 323 & 752 & 163 & 5 & 5 & 6 & $\begin{array}{l}0.00000005 \\
015\end{array}$ & 0.00000002154 & $\begin{array}{l}0.0000000 \\
142\end{array}$ \\
\hline $\begin{array}{l}\text { SERPIN } \\
\text { B3 }\end{array}$ & 6317 & 116 & 3 & $\begin{array}{l}413.333333 \\
333333\end{array}$ & 257 & 764 & 219 & 6 & 5 & 5 & $\begin{array}{l}0.00000000 \\
1054\end{array}$ & 0.00000002768 & $\begin{array}{l}0.0000008 \\
85\end{array}$ \\
\hline $\begin{array}{l}\text { AKR1D } \\
1\end{array}$ & 6718 & 45 & 9 & $\begin{array}{l}414.333333 \\
333333\end{array}$ & 339 & 783 & 121 & 4 & 4 & 6 & $\begin{array}{l}0.00000010 \\
11\end{array}$ & 0.00000004375 & $7.688 \mathrm{e}-11$ \\
\hline ITPA & 3704 & 184 & 19 & $\begin{array}{l}418.666666 \\
686667\end{array}$ & 239 & 856 & 161 & 7 & 5 & 7 & $3.618 \mathrm{e}-10$ & 0.0000002466 & $\begin{array}{l}0.0000000 \\
07813\end{array}$ \\
\hline PROX1 & 5629 & 732 & 10 & 420 & 357 & 412 & 491 & 8 & 14 & 5 & \begin{tabular}{|l}
0.00000020 \\
96 \\
\end{tabular} & $2.803 \mathrm{e}-14$ & 0.002733 \\
\hline BMP7 & 655 & 939 & 12 & $\begin{array}{l}421.666666 \\
666667\end{array}$ & 266 & 712 & 287 & 11 & 10 & 8 & $\begin{array}{l}0.00000000 \\
1438\end{array}$ & 0.000000005798 & $\begin{array}{l}0.0000254 \\
8 \\
\end{array}$ \\
\hline MAPK9 & 5601 & 539 & 9 & 422 & 560 & 387 & 319 & 5 & 13 & 6 & 0.00006903 & $9.625 \mathrm{e}-15$ & $\begin{array}{l}0.0000786 \\
2\end{array}$ \\
\hline $\begin{array}{l}\text { NOTCH } \\
3\end{array}$ & 4854 & 569 & 8 & $\begin{array}{l}422.333333 \\
333333\end{array}$ & 318 & 509 & 440 & 8 & 11 & 5 & $\begin{array}{l}0.00000003 \\
553\end{array}$ & $4.409 \mathrm{e}-12$ & 0.0009662 \\
\hline MUC16 & 94025 & 255 & 3 & 425 & 334 & 648 & 293 & 6 & 7 & 5 & $\begin{array}{l}0.00000008 \\
956\end{array}$ & 0.000000001282 & $\begin{array}{l}0.0000321 \\
6 \\
\end{array}$ \\
\hline LECT2 & 3950 & 84 & 11 & $\begin{array}{l}425.333333 \\
333333\end{array}$ & 300 & 713 & 263 & 5 & 5 & 4 & \begin{tabular}{|l|}
0.00000001 \\
383
\end{tabular} & 0.000000005817 & \begin{tabular}{|l|}
0.0000114 \\
5
\end{tabular} \\
\hline $\begin{array}{l}\text { ANGPT } \\
\text { L2 }\end{array}$ & 23452 & 106 & 11 & $\begin{array}{l}425.666666 \\
666667\end{array}$ & 610 & 226 & 441 & 3 & 13 & 3 & 0.0001428 & $1.047 \mathrm{e}-23$ & 0.0009729 \\
\hline MMP7 & 4316 & 558 & 10 & 426 & 451 & 394 & 433 & 6 & 13 & 5 & 0.00000616 & $1.453 \mathrm{e}-14$ & 0.0008973 \\
\hline TGFA & 7039 & 462 & 9 & $\begin{array}{l}428.666666 \\
666667 \\
\end{array}$ & 289 & 470 & 527 & 8 & 11 & 4 & \begin{tabular}{|l|}
0.00000000 \\
7988 \\
\end{tabular} & $5.328 \mathrm{e}-13$ & 0.004384 \\
\hline CLU & 1191 & 696 & 10 & $\begin{array}{l}428.666666 \\
666667\end{array}$ & 413 & 498 & 375 & 7 & 12 & 6 & $\begin{array}{l}0.00000171 \\
7\end{array}$ & $2.533 \mathrm{e}-12$ & 0.0002749 \\
\hline SAA1 & 6288 & 343 & 13 & $\begin{array}{l}429.333333 \\
333333\end{array}$ & 626 & 198 & 464 & 4 & 19 & 4 & 0.000181 & $9.155 \mathrm{e}-26$ & 0.00162 \\
\hline MACC1 & $\begin{array}{r}34638 \\
9 \\
\end{array}$ & 112 & 9 & $\begin{array}{l}430.666666 \\
666667\end{array}$ & 326 & 517 & 449 & 5 & 7 & 3 & \begin{tabular}{|l|}
0.00000005 \\
44
\end{tabular} & $5.078 \mathrm{e}-12$ & 0.001125 \\
\hline HBEGF & 1839 & 334 & 8 & $\begin{array}{l}432.666666 \\
666667 \\
\end{array}$ & 304 & 533 & 461 & 7 & 9 & 4 & \begin{tabular}{|l}
0.00000001 \\
734 \\
\end{tabular} & $1.236 \mathrm{e}-11$ & 0.001474 \\
\hline CXCL11 & 6373 & 116 & 6 & $\begin{array}{l}434.666666 \\
666667 \\
\end{array}$ & 329 & 522 & 453 & 5 & 7 & 3 & \begin{tabular}{|l|}
0.00000006 \\
428 \\
\end{tabular} & $6.45 \mathrm{e}-12$ & 0.001238 \\
\hline MAT1A & 4143 & 137 & 14 & 436 & 348 & 645 & 315 & 5 & 6 & 4 & $\begin{array}{l}0.00000013 \\
99\end{array}$ & 0.000000001147 & \begin{tabular}{|l}
0.0000672 \\
6
\end{tabular} \\
\hline PTPN1 & 5770 & 541 & 10 & $\begin{array}{l}436.333333 \\
333333\end{array}$ & 562 & 566 & 181 & 5 & 10 & 9 & 0.00006997 & $4.248 \mathrm{e}-11$ & $\begin{array}{l}0.0000000 \\
4796\end{array}$ \\
\hline CXCL13 & 10563 & 241 & 7 & $\begin{array}{l}437.666666 \\
666667\end{array}$ & 330 & 405 & 578 & 6 & 10 & 3 & \begin{tabular}{|l|}
0.00000006 \\
498 \\
\end{tabular} & $2.253 \mathrm{e}-14$ & 0.008269 \\
\hline EPO & 2056 & 792 & 10 & $\begin{array}{l}440.6666666 \\
666667\end{array}$ & 373 & 435 & 514 & 8 & 14 & 5 & $\begin{array}{l}0.00000035 \\
95\end{array}$ & $7.43 \mathrm{e}-14$ & 0.003716 \\
\hline
\end{tabular}


bioRxiv preprint doi: https://doi.org/10.1101/2021.09.06.459063; this version posted September 6, 2021. The copyright holder for this preprint (which was not certified by peer review) is the author/funder. All rights reserved. No reuse allowed without permission.

\begin{tabular}{|c|c|c|c|c|c|c|c|c|c|c|c|c|c|}
\hline LPIN1 & 23175 & 200 & 10 & $\begin{array}{l}441.666666 \\
666667\end{array}$ & 393 & 723 & 209 & 5 & 6 & 6 & $\begin{array}{l}0.00000081 \\
09\end{array}$ & 0.000000009831 & $\begin{array}{l}0.0000003 \\
949\end{array}$ \\
\hline $\begin{array}{l}\text { HSD3B } \\
7\end{array}$ & 80270 & 44 & 8 & 445 & 336 & 779 & 220 & 4 & 4 & 4 & $\begin{array}{l}0.00000009 \\
299 \\
\end{array}$ & 0.00000004011 & $\begin{array}{l}0.0000009 \\
871 \\
\end{array}$ \\
\hline $\begin{array}{l}\text { СYP8В } \\
1 \\
\end{array}$ & 1582 & 39 & 12 & 449 & 468 & 762 & 117 & 3 & 4 & 6 & \begin{tabular}{|l|}
0.00000904 \\
2 \\
\end{tabular} & 0.00000002489 & $3.2 \mathrm{e}-11$ \\
\hline RORA & 6095 & 428 & 9 & $\begin{array}{l}449.666666 \\
666667\end{array}$ & 515 & 456 & 378 & 5 & 11 & 5 & 0.00002508 & $2.439 \mathrm{e}-13$ & 0.0002977 \\
\hline SCTR & 6344 & 79 & 9 & $\begin{array}{l}450.333333 \\
333333\end{array}$ & 396 & 695 & 260 & 4 & 5 & 4 & $\begin{array}{l}0.00000085 \\
86\end{array}$ & 0.000000004369 & $\begin{array}{l}0.0000090 \\
45\end{array}$ \\
\hline NGF & 4803 & 820 & 9 & $\begin{array}{l}450.666666 \\
666667\end{array}$ & 440 & 389 & 523 & 7 & 15 & 5 & 0.00000466 & $1.038 \mathrm{e}-14$ & 0.004264 \\
\hline NCF1 & $\begin{array}{r}65336 \\
1 \\
\end{array}$ & 505 & 10 & $\begin{array}{l}451.333333 \\
333333 \\
\end{array}$ & 544 & 261 & 549 & 5 & 18 & 4 & 0.00005222 & $1.911 \mathrm{e}-21$ & 0.005862 \\
\hline PCK1 & 5105 & 352 & 11 & 452 & 638 & 545 & 173 & 4 & 9 & 8 & 0.0001962 & $1.915 \mathrm{e}-11$ & $\begin{array}{l}0.0000000 \\
282\end{array}$ \\
\hline $\begin{array}{l}\text { VPS33 } \\
\text { B }\end{array}$ & 26276 & 75 & 11 & $\begin{array}{l}458.666666 \\
666667\end{array}$ & 388 & 873 & 115 & 4 & 4 & 7 & 0.00000071 & 0.0000003155 & $1.909 \mathrm{e}-11$ \\
\hline CD1D & 912 & 560 & 9 & 460 & 569 & 235 & 576 & 5 & 20 & 4 & 0.00008135 & $3.716 \mathrm{e}-23$ & 0.008182 \\
\hline BLVRA & 644 & 84 & 10 & 461 & 399 & 579 & 405 & 4 & 6 & 3 & $\begin{array}{l}0.00000109 \\
1\end{array}$ & $6.548 \mathrm{e}-11$ & 0.000529 \\
\hline PLIN2 & 123 & 204 & 10 & $\begin{array}{l}462.6666666 \\
666667 \\
\end{array}$ & 397 & 727 & 264 & 5 & 6 & 5 & \begin{tabular}{|l}
0.00000088 \\
51 \\
\end{tabular} & 0.000000011 & $\begin{array}{l}0.0000120 \\
2\end{array}$ \\
\hline $\begin{array}{l}\text { HMGC } \\
\text { S1 }\end{array}$ & 3157 & 161 & 22 & $\begin{array}{l}463.666666 \\
666667\end{array}$ & 367 & 831 & 193 & 5 & 5 & 6 & $\begin{array}{l}0.00000029 \\
65\end{array}$ & 0.0000001309 & $\begin{array}{l}0.0000001 \\
181\end{array}$ \\
\hline $\begin{array}{l}\text { CDKN2 } \\
\text { A }\end{array}$ & 1029 & 3062 & 9 & 465 & 328 & 493 & 574 & 16 & 23 & 9 & \begin{tabular}{|l|}
0.00000006 \\
382 \\
\end{tabular} & $2.246 \mathrm{e}-12$ & 0.007832 \\
\hline CD69 & 969 & 183 & 30 & $\begin{array}{l}465.666666 \\
666667\end{array}$ & 504 & 373 & 520 & 4 & 10 & 3 & 0.00001932 & $1.561 \mathrm{e}-15$ & 0.004127 \\
\hline TAT & 6898 & 123 & 18 & 468 & 267 & 966 & 171 & 6 & 4 & 6 & $\begin{array}{l}0.00000000 \\
1446\end{array}$ & 0.000002082 & $\begin{array}{l}0.0000000 \\
2655\end{array}$ \\
\hline EPHX2 & 2053 & 264 & 13 & $\begin{array}{l}468.666666 \\
666667 \\
\end{array}$ & 563 & 426 & 417 & 4 & 10 & 4 & 0.00007287 & $5.291 \mathrm{e}-14$ & 0.000661 \\
\hline ITGB2 & 3689 & 856 & 9 & $\begin{array}{l}469.3333333 \\
333333 \\
\end{array}$ & 548 & 323 & 537 & 6 & 18 & 5 & 0.00005512 & $1.254 \mathrm{e}-17$ & 0.005024 \\
\hline HLA-G & 3135 & 819 & 5 & $\begin{array}{l}472.666666 \\
666667 \\
\end{array}$ & 438 & 659 & 321 & 7 & 10 & 7 & $\begin{array}{l}0.00000464 \\
5\end{array}$ & 0.0000000001787 & $\begin{array}{l}0.0000830 \\
1\end{array}$ \\
\hline $\begin{array}{l}\text { NOTCH } \\
4 \\
\end{array}$ & 4855 & 335 & 6 & 473 & 619 & 469 & 331 & 4 & 10 & 5 & 0.000167 & $5.006 \mathrm{e}-13$ & 0.0001061 \\
\hline EDNRA & 1909 & 476 & 10 & $\begin{array}{l}473.666666 \\
666667\end{array}$ & 353 & 534 & 534 & 7 & 10 & 4 & $\begin{array}{l}0.00000016 \\
33\end{array}$ & $1.324 \mathrm{e}-11$ & 0.004836 \\
\hline ACE2 & 59272 & 461 & 11 & 474 & 525 & 371 & 526 & 5 & 13 & 4 & 0.00003507 & $1.423 \mathrm{e}-15$ & 0.004359 \\
\hline ABCG5 & 64240 & 210 & 12 & $\begin{array}{l}480.666666 \\
666667\end{array}$ & 752 & 609 & 81 & 3 & 7 & 11 & 0.0008793 & $3.563 \mathrm{e}-10$ & $1.788 \mathrm{e}-14$ \\
\hline $\begin{array}{l}\text { HSP90 } \\
\text { B1 }\end{array}$ & 7184 & 509 & 15 & 481 & 545 & 485 & 413 & 5 & 11 & 5 & 0.0000541 & $1.436 \mathrm{e}-12$ & 0.0006139 \\
\hline PDGFC & 56034 & 129 & 10 & $\begin{array}{l}481.3333333 \\
333333\end{array}$ & 341 & 638 & 465 & 5 & 6 & 3 & $\begin{array}{l}0.00000010 \\
57 \\
\end{array}$ & $8.068 \mathrm{e}-10$ & 0.001651 \\
\hline CCL25 & 6370 & 95 & 7 & 484 & 587 & 588 & 277 & 3 & 6 & 4 & 0.0001069 & $1.369 \mathrm{e}-10$ & $\begin{array}{l}0.0000178 \\
1\end{array}$ \\
\hline CD34 & 947 & 524 & 9 & 485 & 307 & 832 & 316 & 8 & 7 & 6 & $\begin{array}{l}0.00000001 \\
969\end{array}$ & 0.0000001314 & $\begin{array}{l}0.0000677 \\
3\end{array}$ \\
\hline FECH & 2235 & 229 & 20 & 488 & 409 & 909 & 146 & 5 & 5 & 8 & \begin{tabular}{|l}
0.00000151 \\
8 \\
\end{tabular} & 0.0000006829 & $\begin{array}{l}0.0000000 \\
01166\end{array}$ \\
\hline DMBT1 & 1755 & 135 & 8 & $\begin{array}{l}488.666666 \\
666667\end{array}$ & 452 & 543 & 471 & 4 & 7 & 3 & $\begin{array}{l}0.00000644 \\
9\end{array}$ & $1.814 \mathrm{e}-11$ & 0.001865 \\
\hline $\mathrm{FGF} 23$ & 8074 & 594 & 11 & 490 & 384 & 749 & 337 & 7 & 8 & 6 & $\begin{array}{l}0.00000065 \\
32\end{array}$ & 0.00000002088 & 0.0001278 \\
\hline ADH1C & 126 & 316 & 10 & $\begin{array}{l}492.3333333 \\
333333\end{array}$ & 456 & 701 & 320 & 5 & 7 & 5 & $\begin{array}{l}0.00000656 \\
4 \\
\end{array}$ & 0.000000005158 & $\begin{array}{l}0.0000829 \\
6\end{array}$ \\
\hline $\begin{array}{l}\text { CYP27 } \\
\text { A1 }\end{array}$ & 1593 & 179 & 18 & $\begin{array}{l}497.666666 \\
666667\end{array}$ & 716 & 703 & 74 & 3 & 6 & 11 & 0.0005771 & 0.000000005224 & 3.36e-15 \\
\hline LOXL2 & 4017 & 141 & 11 & $\begin{array}{l}498.3333333 \\
333333\end{array}$ & 462 & 553 & 480 & 4 & 7 & 3 & $\begin{array}{l}0.00000750 \\
4 \\
\end{array}$ & $2.421 \mathrm{e}-11$ & 0.00208 \\
\hline ADD3 & 120 & 93 & 10 & 499 & 311 & 914 & 272 & 5 & 4 & 4 & $\begin{array}{l}0.00000002 \\
233\end{array}$ & 0.0000007179 & $\begin{array}{l}0.0000166 \\
5\end{array}$ \\
\hline $\begin{array}{l}\text { HAVCR } \\
1 \\
\end{array}$ & 26762 & 180 & 3 & 501 & 299 & 852 & 352 & 6 & 5 & 4 & $\begin{array}{l}0.00000001 \\
269\end{array}$ & 0.0000002221 & 0.0001765 \\
\hline PGF & 5228 & 415 & 9 & $\begin{array}{l}502.3333333 \\
333333 \\
\end{array}$ & 680 & 328 & 499 & 4 & 14 & 4 & 0.0003464 & $1.773 \mathrm{e}-17$ & 0.003099 \\
\hline ARNT & 405 & 380 & 9 & $\begin{array}{l}503.3333333 \\
333333\end{array}$ & 391 & 636 & 483 & 6 & 8 & 4 & \begin{tabular}{|l}
0.00000078 \\
66 \\
\end{tabular} & $8.05 \mathrm{e}-10$ & 0.002296 \\
\hline $\begin{array}{l}\text { CYP1A } \\
2 \\
\end{array}$ & 1544 & 639 & 8 & 504 & 799 & 433 & 280 & 4 & 13 & 7 & 0.001504 & $7.053 \mathrm{e}-14$ & $\begin{array}{l}0.0000195 \\
3\end{array}$ \\
\hline $\begin{array}{l}\text { BCL2L1 } \\
1 \\
\end{array}$ & 10018 & 832 & 6 & $\begin{array}{l}505.3333333 \\
333333\end{array}$ & 539 & 448 & 529 & 6 & 14 & 5 & 0.00004795 & $1.368 \mathrm{e}-13$ & 0.004497 \\
\hline APLNR & 187 & 168 & 7 & 506 & 488 & 688 & 342 & 4 & 6 & 4 & 0.00001423 & 0.000000003654 & 0.0001385 \\
\hline $\begin{array}{l}\text { MUC5 } \\
\text { AC }\end{array}$ & 4586 & 422 & 9 & $\begin{array}{l}507.666666 \\
666667 \\
\end{array}$ & 686 & 332 & 505 & 4 & 14 & 4 & 0.0003659 & $2.2 e-17$ & 0.00326 \\
\hline DKK1 & 22943 & 820 & 9 & $\begin{array}{l}509.666666 \\
666667\end{array}$ & 375 & 742 & 412 & 8 & 9 & 6 & $\begin{array}{l}0.00000046 \\
2\end{array}$ & 0.00000001947 & 0.0006083 \\
\hline $\begin{array}{l}\text { PSMD1 } \\
0\end{array}$ & 5716 & 160 & 17 & $\begin{array}{l}512.333333 \\
333333\end{array}$ & 366 & 676 & 495 & 5 & 6 & 3 & $\begin{array}{l}0.00000028 \\
82\end{array}$ & 0.000000002778 & 0.002926 \\
\hline S1PR2 & 9294 & 225 & 10 & 516 & 535 & 624 & 389 & 4 & 7 & 4 & 0.00004103 & $5.609 \mathrm{e}-10$ & 0.0003815 \\
\hline CD5L & 922 & 63 & 6 & 516 & 524 & 656 & 368 & 3 & 5 & 3 & 0.00003477 & 0.000000001457 & 0.0002453 \\
\hline
\end{tabular}


bioRxiv preprint doi: https://doi.org/10.1101/2021.09.06.459063; this version posted September 6, 2021. The copyright holder for this preprint (which was not certified by peer review) is the author/funder. All rights reserved. No reuse allowed without permission.

\begin{tabular}{|c|c|c|c|c|c|c|c|c|c|c|c|c|c|}
\hline CES1 & 1066 & 146 & 20 & $\begin{array}{l}520.666666 \\
666667 \\
\end{array}$ & 677 & 562 & 323 & 3 & 7 & 4 & 0.0003341 & $3.044 \mathrm{e}-11$ & $\begin{array}{l}0.0000843 \\
7\end{array}$ \\
\hline MERTK & 10461 & 270 & 10 & 522 & 567 & 577 & 422 & 4 & 8 & 4 & 0.00007898 & $6.147 \mathrm{e}-11$ & 0.0007126 \\
\hline ACACB & 32 & 138 & 10 & 522 & 457 & 791 & 318 & 4 & 5 & 4 & $\begin{array}{l}0.00000696 \\
5\end{array}$ & 0.00000006381 & 0.0000686 \\
\hline ACOX3 & 8310 & 86 & 15 & 522 & 404 & 897 & 265 & 4 & 4 & 4 & $\begin{array}{l}0.00000118 \\
5\end{array}$ & 0.0000005339 & $\begin{array}{l}0.0000124 \\
8\end{array}$ \\
\hline UCP2 & 7351 & 684 & 12 & $\begin{array}{l}527.666666 \\
666667 \\
\end{array}$ & 822 & 558 & 203 & 4 & 11 & 9 & 0.001882 & $2.722 \mathrm{e}-11$ & $\begin{array}{l}0.0000003 \\
058\end{array}$ \\
\hline $\mathrm{CD} 24$ & $\begin{array}{r}10013 \\
3941 \\
\end{array}$ & 250 & & 533 & 419 & 773 & 407 & 5 & 6 & 4 & $\begin{array}{l}0.00000227 \\
9\end{array}$ & 0.00000003445 & 0.0005485 \\
\hline IGFBP1 & 3484 & 434 & 11 & 541 & 335 & 778 & 510 & 7 & 7 & 4 & $\begin{array}{l}0.00000009 \\
262 \\
\end{array}$ & 0.00000003988 & 0.003587 \\
\hline $\mathrm{CTH}$ & 1491 & 239 & 20 & $\begin{array}{l}547.666666 \\
666667 \\
\end{array}$ & 780 & 640 & 223 & 3 & 7 & 6 & 0.001239 & $8.299 \mathrm{e}-10$ & $\begin{array}{l}0.0000010 \\
56\end{array}$ \\
\hline $\begin{array}{l}\text { AGPAT } \\
4\end{array}$ & 56895 & 51 & 10 & $\begin{array}{l}548.666666 \\
666667 \\
\end{array}$ & 503 & 800 & 343 & 3 & 4 & 3 & 0.00001908 & 0.00000007163 & 0.0001389 \\
\hline TGFB3 & 7043 & 549 & 9 & $\begin{array}{l}550.666666 \\
666667 \\
\end{array}$ & 446 & 634 & 572 & 6 & 9 & 4 & $\begin{array}{l}0.00000568 \\
1\end{array}$ & $7.745 \mathrm{e}-10$ & 0.007658 \\
\hline ACACA & 31 & 347 & 20 & 552 & 472 & 849 & 335 & 5 & 6 & 5 & $\begin{array}{l}0.00000997 \\
7\end{array}$ & 0.0000002138 & 0.000124 \\
\hline GLUL & 2752 & 405 & 24 & $\begin{array}{l}553.333333 \\
333333 \\
\end{array}$ & 401 & 893 & 366 & 6 & 6 & 5 & $\begin{array}{l}0.00000110 \\
8\end{array}$ & 0.0000004978 & 0.0002369 \\
\hline PTPRO & 5800 & 108 & 8 & $\begin{array}{l}553.666666 \\
666667 \\
\end{array}$ & 426 & 945 & 290 & 4 & 4 & 4 & $\begin{array}{l}0.00000280 \\
7\end{array}$ & 0.000001266 & $\begin{array}{l}0.0000283 \\
9\end{array}$ \\
\hline NOX1 & 27035 & 485 & 14 & $\begin{array}{l}559.666666 \\
666667 \\
\end{array}$ & 537 & 603 & 539 & 5 & 9 & 4 & 0.00004365 & $2.809 \mathrm{e}-10$ & 0.00514 \\
\hline BHMT & 635 & 159 & 10 & $\begin{array}{l}561.666666 \\
666667 \\
\end{array}$ & 477 & 1018 & 190 & 4 & 4 & 6 & 0.00001171 & 0.000005487 & $\begin{array}{l}0.0000001 \\
113\end{array}$ \\
\hline DGUOK & 1716 & 68 & 12 & $\begin{array}{l}562.3333333 \\
333333\end{array}$ & 378 & 1124 & 185 & 4 & 3 & 5 & $\begin{array}{l}0.00000049 \\
01\end{array}$ & 0.00002042 & $\begin{array}{l}0.0000000 \\
7057\end{array}$ \\
\hline LOX & 4015 & 322 & 10 & 564 & 248 & 987 & 457 & 8 & 5 & 4 & $5.691 \mathrm{e}-10$ & 0.000003322 & 0.001293 \\
\hline ALPL & 249 & 530 & 12 & $\begin{array}{l}564.666666 \\
666667 \\
\end{array}$ & 215 & 1403 & 76 & 10 & 4 & 15 & $9.2 \mathrm{e}-11$ & 0.000425 & $5.084 \mathrm{e}-15$ \\
\hline PHB & 5245 & 409 & 23 & \begin{tabular}{|l|}
565.666666 \\
666667 \\
\end{tabular} & 675 & 653 & 369 & 4 & 8 & 5 & 0.0003301 & 0.000000001385 & 0.0002461 \\
\hline $\mathrm{XDH}$ & 7498 & 978 & 18 & $\begin{array}{l}569.333333 \\
333333 \\
\end{array}$ & 743 & 719 & 246 & 5 & 10 & 9 & 0.0008134 & 0.000000008313 & $\begin{array}{l}0.0000046 \\
65\end{array}$ \\
\hline F5 & 2153 & 1275 & 10 & $\begin{array}{l}569.666666 \\
666667 \\
\end{array}$ & 553 & 1001 & 155 & 7 & 8 & 14 & 0.00005947 & 0.000004151 & $\begin{array}{l}0.00000000 \\
03234\end{array}$ \\
\hline CAV2 & 858 & 182 & 10 & 570 & 502 & 853 & 355 & 4 & 5 & 4 & 0.00001898 & 0.0000002344 & 0.0001827 \\
\hline SREBF2 & 6721 & 357 & 10 & $\begin{array}{l}572.666666 \\
666667 \\
\end{array}$ & 885 & 619 & 214 & 3 & 8 & 7 & 0.003499 & $5.095 \mathrm{e}-10$ & $\begin{array}{l}0.0000005 \\
474\end{array}$ \\
\hline GLS & 2744 & 192 & 15 & $\begin{array}{l}581.666666 \\
666667\end{array}$ & 512 & 869 & 364 & 4 & 5 & 4 & 0.00002299 & 0.0000002997 & 0.0002197 \\
\hline $\begin{array}{l}\text { ATP11 } \\
\text { C }\end{array}$ & $\begin{array}{r}28641 \\
0 \\
\end{array}$ & 46 & 10 & $\begin{array}{l}583.3333333 \\
333333\end{array}$ & 490 & 1035 & 225 & 3 & 3 & 4 & 0.00001431 & 0.000006773 & $\begin{array}{l}0.0000011 \\
59\end{array}$ \\
\hline $\mathrm{GC}$ & 2638 & 365 & 10 & $\begin{array}{l}584.3333333 \\
333333 \\
\end{array}$ & 383 & 1025 & 345 & 6 & 5 & 5 & $\begin{array}{l}0.00000063 \\
6\end{array}$ & 0.000005863 & 0.0001533 \\
\hline MCAR & 4160 & 652 & 10 & 585 & 615 & 781 & 359 & 5 & 8 & 6 & 0.0001535 & 0.00000004036 & 0.0002007 \\
\hline PDCD4 & 27250 & 350 & 15 & 588 & 881 & 415 & 468 & 3 & 11 & 4 & 0.00332 & $3.112 \mathrm{e}-14$ & 0.001735 \\
\hline FOXO1 & 2308 & 1066 & 11 & \begin{tabular}{|l|}
588.333333 \\
333333 \\
\end{tabular} & 617 & 838 & 310 & 6 & 9 & 8 & 0.0001607 & 0.0000001478 & $\begin{array}{l}0.0000577 \\
7 \\
\end{array}$ \\
\hline отс & 5009 & 273 & 15 & \begin{tabular}{|l}
589.666666 \\
666667 \\
\end{tabular} & 431 & 1179 & 159 & 5 & 4 & 8 & $\begin{array}{l}0.00000340 \\
7\end{array}$ & 0.00003964 & $\begin{array}{l}0.00000000 \\
04259\end{array}$ \\
\hline PON1 & 5444 & 1181 & 7 & $\begin{array}{l}590.333333 \\
333333 \\
\end{array}$ & 652 & 705 & 414 & 6 & 11 & 7 & 0.0002624 & 0.000000005273 & 0.0006134 \\
\hline HPX & 3263 & 138 & 10 & 594 & 661 & 646 & 475 & 3 & 6 & 3 & 0.0002893 & 0.000000001196 & 0.001973 \\
\hline FABP4 & 2167 & 392 & 14 & $\begin{array}{l}596.3333333 \\
333333\end{array}$ & 659 & 643 & 487 & 4 & 8 & 4 & 0.0002873 & 0.000000001013 & 0.002561 \\
\hline KLB & $\begin{array}{r}15283 \\
1 \\
\end{array}$ & 72 & 9 & $\begin{array}{l}596.666666 \\
666667 \\
\end{array}$ & 543 & 865 & 382 & 3 & 4 & 3 & 0.00005023 & 0.0000002701 & 0.0003533 \\
\hline $\begin{array}{l}\text { ENTPD } \\
2 \\
\end{array}$ & 954 & 96 & 12 & $\begin{array}{l}597.666666 \\
666667\end{array}$ & 589 & 926 & 278 & 3 & 4 & 4 & 0.0001099 & 0.0000008052 & 0.0000185 \\
\hline MX1 & 4599 & 258 & 13 & $\begin{array}{l}600.3333333 \\
333333 \\
\end{array}$ & 558 & 652 & 591 & 4 & 7 & 3 & 0.00006721 & 0.000000001381 & 0.009842 \\
\hline OCLN & $\begin{array}{r}10050 \\
6658 \\
\end{array}$ & 410 & 9 & $\begin{array}{l}603.666666 \\
666667 \\
\end{array}$ & 676 & 765 & 370 & 4 & 7 & 5 & 0.0003325 & 0.00000002765 & 0.0002483 \\
\hline HLA-B & 3106 & 1908 & 48 & $\begin{array}{l}607.3333333 \\
333333 \\
\end{array}$ & 354 & 1071 & 397 & 12 & 9 & 9 & $\begin{array}{l}0.00000017 \\
04\end{array}$ & 0.00001072 & 0.0004669 \\
\hline $\begin{array}{l}\text { CYP4B } \\
1\end{array}$ & 1580 & 78 & 13 & $\begin{array}{l}611.3333333 \\
333333\end{array}$ & 556 & 883 & 395 & 3 & 4 & 3 & 0.00006242 & 0.0000003658 & 0.0004345 \\
\hline $\begin{array}{l}\text { SLC39A } \\
14\end{array}$ & 23516 & 93 & 12 & $\begin{array}{l}612.666666 \\
666667\end{array}$ & 936 & 484 & 418 & 2 & 7 & 3 & 0.005236 & $1.439 \mathrm{e}-12$ & 0.000695 \\
\hline ATF4 & 468 & 497 & 11 & \begin{tabular}{|l|l}
613.666666 \\
666667 \\
\end{tabular} & 722 & 814 & 305 & 4 & 7 & 6 & 0.0006479 & 0.00000009446 & $\begin{array}{l}0.0000521 \\
6\end{array}$ \\
\hline MTHFR & 4524 & 3355 & 20 & $\begin{array}{l}618.3333333 \\
333333 \\
\end{array}$ & 382 & 1273 & 200 & 15 & 10 & 19 & $\begin{array}{l}0.00000061 \\
36\end{array}$ & 0.0001294 & $\begin{array}{l}0.0000002 \\
565\end{array}$ \\
\hline FUT2 & 2524 & 167 & 10 & 619 & 485 & 1033 & 339 & 4 & 4 & 4 & 0.00001399 & 0.000006566 & 0.0001365 \\
\hline ELOVL6 & 79071 & 122 & 14 & $\begin{array}{l}619.333333 \\
333333\end{array}$ & 435 & 964 & 459 & 4 & 4 & 3 & $\begin{array}{l}0.00000447 \\
6\end{array}$ & 0.00000202 & 0.001418 \\
\hline $\begin{array}{l}\text { HMGC } \\
\mathrm{R}\end{array}$ & 3156 & 483 & 24 & 623 & 719 & 949 & 201 & 4 & 6 & 8 & 0.0005837 & 0.000001288 & $\begin{array}{l}0.0000002 \\
727\end{array}$ \\
\hline
\end{tabular}


bioRxiv preprint doi: https://doi.org/10.1101/2021.09.06.459063; this version posted September 6, 2021. The copyright holder for this preprint (which was not certified by peer review) is the author/funder. All rights reserved. No reuse allowed without permission.

\begin{tabular}{|c|c|c|c|c|c|c|c|c|c|c|c|c|c|}
\hline $\mathrm{AHCY}$ & 191 & 226 & 22 & \begin{tabular}{|l}
624.666666 \\
666667 \\
\end{tabular} & 536 & 1120 & 218 & 4 & 4 & 6 & 0.00004168 & 0.00001995 & $\begin{array}{l}0.0000007 \\
781\end{array}$ \\
\hline TLR8 & 51311 & 258 & 11 & 625 & 800 & 483 & 592 & 3 & 9 & 3 & 0.00151 & $1.391 \mathrm{e}-12$ & 0.009825 \\
\hline$T \pi R$ & 7276 & 745 & 10 & $\begin{array}{l}629.3333333 \\
333333 \\
\end{array}$ & 310 & 1082 & 496 & 9 & 6 & 5 & $\begin{array}{l}0.00000002 \\
096\end{array}$ & 0.00001299 & 0.002928 \\
\hline ITGA1 & 3672 & 247 & 9 & 630 & 551 & 936 & 403 & 4 & 5 & 4 & 0.00005741 & 0.0000009615 & 0.0005287 \\
\hline ASS1 & 445 & 248 & 20 & 630 & 552 & 1155 & 183 & 4 & 4 & 7 & 0.00005823 & 0.00002783 & $\begin{array}{l}0.0000000 \\
5389\end{array}$ \\
\hline DLAT & 1737 & 162 & 24 & 631 & 370 & 1026 & 497 & 5 & 4 & 3 & $\begin{array}{l}0.00000030 \\
32\end{array}$ & 0.000005862 & 0.003023 \\
\hline $\begin{array}{l}\text { HLA- } \\
\text { DQA1 }\end{array}$ & 3117 & 905 & 7 & 633 & 292 & 1168 & 439 & 10 & 6 & 6 & $\begin{array}{l}0.00000001 \\
01\end{array}$ & 0.00003506 & 0.0009671 \\
\hline TFF3 & 7033 & 209 & 8 & 634 & 749 & 608 & 545 & 3 & 7 & 3 & 0.0008706 & $3.453 \mathrm{e}-10$ & 0.005801 \\
\hline FOXL1 & 2300 & 56 & 7 & 641 & 514 & 816 & 593 & 3 & 4 & 2 & 0.00002481 & 0.000000103 & 0.009944 \\
\hline $\begin{array}{l}\text { EHHAD } \\
\mathrm{H}\end{array}$ & 1962 & 83 & 12 & $\begin{array}{l}644.666666 \\
666667 \\
\end{array}$ & 564 & 1173 & 197 & 3 & 3 & 5 & 0.0000742 & 0.00003568 & $\begin{array}{l}0.0000001 \\
825\end{array}$ \\
\hline ERRFI1 & 54206 & 140 & 11 & $\begin{array}{l}646.666666 \\
666667 \\
\end{array}$ & 665 & 797 & 478 & 3 & 5 & 3 & 0.0003001 & 0.00000006804 & 0.002046 \\
\hline TYMP & 1890 & 210 & 6 & 653 & 523 & 889 & 547 & 4 & 5 & 3 & 0.00003202 & 0.000000456 & 0.005862 \\
\hline SHBG & 6462 & 337 & 9 & $\begin{array}{l}657.3333333 \\
333333 \\
\end{array}$ & 872 & 845 & 255 & 3 & 6 & 6 & 0.003009 & 0.0000001812 & $\begin{array}{l}0.0000068 \\
16 \\
\end{array}$ \\
\hline SLC51B & $\begin{array}{r}12326 \\
4 \\
\end{array}$ & 38 & 7 & $\begin{array}{l}660.666666 \\
666667 \\
\end{array}$ & 465 & 1445 & 72 & 3 & 2 & 8 & $\begin{array}{l}0.00000840 \\
2 \\
\end{array}$ & 0.0005655 & $8.532 \mathrm{e}-16$ \\
\hline $\begin{array}{l}\text { SLC25A } \\
13\end{array}$ & 10165 & 143 & 8 & $\begin{array}{l}661.666666 \\
666667 \\
\end{array}$ & 669 & 1296 & 20 & 3 & 3 & 24 & 0.0003176 & 0.0001639 & $1.398 \mathrm{e}-39$ \\
\hline GYS2 & 2998 & 71 & 9 & 663 & 878 & 859 & 252 & 2 & 4 & 4 & 0.00326 & 0.0000002569 & $\begin{array}{l}0.0000060 \\
66\end{array}$ \\
\hline CDK1 & 983 & 1289 & 13 & 666 & 557 & 905 & 536 & 7 & 9 & 6 & 0.00006312 & 0.0000006245 & 0.004946 \\
\hline EZR & 7430 & 681 & 11 & 671 & 628 & 908 & 477 & 5 & 7 & 5 & 0.000184 & 0.0000006776 & 0.002029 \\
\hline INSR & 3643 & 1129 & 11 & \begin{tabular}{|l|}
679.666666 \\
666667 \\
\end{tabular} & 1026 & 615 & 398 & 4 & 12 & 7 & 0.009237 & $4.228 \mathrm{e}-10$ & 0.0004863 \\
\hline UTP4 & 84916 & 94 & 19 & \begin{tabular}{|l|}
685.333333 \\
333333 \\
\end{tabular} & 585 & 1196 & 275 & 3 & 3 & 4 & 0.0001039 & 0.00005084 & $\begin{array}{l}0.0000171 \\
9 \\
\end{array}$ \\
\hline SMN1 & 6606 & 597 & 11 & $\begin{array}{l}687.3333333 \\
333333 \\
\end{array}$ & 466 & 999 & 597 & 6 & 6 & 4 & $\begin{array}{l}0.00000873 \\
7\end{array}$ & 0.000004075 & 0.009979 \\
\hline $\begin{array}{l}\text { TRAPP } \\
\text { C11 }\end{array}$ & 60684 & 72 & 15 & $\begin{array}{l}687.666666 \\
666667 \\
\end{array}$ & 542 & 1140 & 381 & 3 & 3 & 3 & 0.00005032 & 0.00002392 & 0.0003543 \\
\hline TNNI3 & 7137 & 513 & 8 & $\begin{array}{l}687.6666666 \\
666667 \\
\end{array}$ & 362 & 1149 & 552 & 7 & 5 & 4 & $\begin{array}{l}0.00000026 \\
23\end{array}$ & 0.00002663 & 0.006178 \\
\hline PRDX4 & 10549 & 177 & 11 & $\begin{array}{l}690.3333333 \\
333333 \\
\end{array}$ & 500 & 1055 & 516 & 4 & 4 & 3 & 0.00001708 & 0.000008093 & 0.003774 \\
\hline SLC2A2 & 6514 & 335 & 10 & $\begin{array}{l}691.666666 \\
666667 \\
\end{array}$ & 870 & 997 & 208 & 3 & 5 & 7 & 0.002964 & 0.000003983 & $\begin{array}{l}0.0000003 \\
668\end{array}$ \\
\hline APOB & 338 & 1023 & 9 & 697 & 499 & 1215 & 377 & 7 & 6 & 7 & 0.00001678 & 0.00006544 & 0.0002817 \\
\hline LUM & 4060 & 191 & 10 & $\begin{array}{l}704.6666666 \\
666667\end{array}$ & 511 & 1072 & 531 & 4 & 4 & 3 & 0.00002256 & 0.00001075 & 0.00458 \\
\hline RPLPO & 6175 & 338 & 22 & \begin{tabular}{|l}
705.666666 \\
666667 \\
\end{tabular} & 624 & 1236 & 257 & 4 & 4 & 6 & 0.0001715 & 0.0000866 & $\begin{array}{l}0.0000068 \\
79\end{array}$ \\
\hline $\mathrm{BCHE}$ & 590 & 303 & 11 & $\begin{array}{l}706.3333333 \\
333333 \\
\end{array}$ & 596 & 1206 & 317 & 4 & 4 & 5 & 0.0001176 & 0.00005812 & $\begin{array}{l}0.0000684 \\
4\end{array}$ \\
\hline FOXA1 & 3169 & 421 & 9 & $\begin{array}{l}707.666666 \\
666667 \\
\end{array}$ & 938 & 903 & 282 & 3 & 6 & 6 & 0.0053 & 0.0000006153 & $\begin{array}{l}0.0000220 \\
5\end{array}$ \\
\hline GPX4 & 2879 & 261 & 12 & 713 & 561 & 1163 & 415 & 4 & 4 & 4 & 0.00006992 & 0.00003373 & 0.0006355 \\
\hline STK25 & 10494 & 87 & 11 & $\begin{array}{l}721.333333 \\
333333 \\
\end{array}$ & 573 & 1181 & 410 & 3 & 3 & 3 & 0.00008413 & 0.00004082 & 0.0005807 \\
\hline ITGB4 & 3691 & 399 & 10 & 723 & 922 & 757 & 490 & 3 & 7 & 4 & 0.004624 & 0.00000002326 & 0.002721 \\
\hline XBP1 & 7494 & 458 & 5 & $\begin{array}{l}724.6666666 \\
666667 \\
\end{array}$ & 969 & 681 & 524 & 3 & 8 & 4 & 0.006521 & 0.000000003172 & 0.004271 \\
\hline LTBP3 & 4054 & 91 & 8 & 728 & 581 & 1187 & 416 & 3 & 3 & 3 & 0.00009496 & 0.00004648 & 0.0006542 \\
\hline GPX1 & 2876 & 705 & 11 & $\begin{array}{l}730.666666 \\
666667 \\
\end{array}$ & 643 & 1065 & 484 & 5 & 6 & 5 & 0.000211 & 0.000009709 & 0.00234 \\
\hline RARA & 5914 & 802 & 9 & 735 & 683 & 1116 & 406 & 5 & 6 & 6 & 0.0003598 & 0.00001893 & 0.0005479 \\
\hline GSTT1 & 2952 & 1711 & & $\begin{array}{l}741.333333 \\
333333 \\
\end{array}$ & 550 & 1152 & 522 & 8 & 8 & 7 & 0.00005706 & 0.00002724 & 0.004205 \\
\hline $\begin{array}{l}\text { CYP3A } \\
7 \\
\end{array}$ & 1551 & 189 & 10 & \begin{tabular}{|l|}
748.666666 \\
666667 \\
\end{tabular} & 510 & 1374 & 362 & 4 & 3 & 4 & 0.00002169 & 0.000353 & 0.0002077 \\
\hline $\mathrm{NPC2}$ & 10577 & 171 & 12 & 751 & 706 & 1040 & 507 & 3 & 4 & 3 & 0.0005113 & 0.000007163 & 0.003472 \\
\hline HPD & 3242 & 104 & 17 & $\begin{array}{l}753.666666 \\
666667 \\
\end{array}$ & 607 & 1219 & 435 & 3 & 3 & 3 & 0.0001356 & 0.0000675 & 0.0009319 \\
\hline $\begin{array}{l}\text { ONECU } \\
\text { T2 }\end{array}$ & 9480 & 59 & 8 & 764 & 845 & 1086 & 361 & 2 & 3 & 3 & 0.002341 & 0.00001376 & 0.0002052 \\
\hline $\begin{array}{l}\text { SLC22A } \\
1\end{array}$ & 6580 & 196 & 7 & $\begin{array}{l}778.3333333 \\
333333\end{array}$ & 735 & 1393 & 207 & 3 & 3 & 6 & 0.0007344 & 0.0003875 & $\begin{array}{l}0.0000003 \\
539\end{array}$ \\
\hline DCLK1 & 9201 & 154 & 6 & 779 & 687 & 1321 & 329 & 3 & 3 & 4 & 0.0003856 & 0.0002005 & 0.0001022 \\
\hline $\begin{array}{l}\text { TMEM } \\
30 \mathrm{~A} \\
\end{array}$ & 55754 & 75 & 22 & $\begin{array}{l}791.333333 \\
333333 \\
\end{array}$ & 549 & 1634 & 191 & 3 & 2 & 5 & 0.0000562 & 0.001955 & $\begin{array}{l}0.0000001 \\
126\end{array}$ \\
\hline
\end{tabular}


bioRxiv preprint doi: https://doi.org/10.1101/2021.09.06.459063; this version posted September 6, 2021. The copyright holder for this preprint (which was not certified by peer review) is the author/funder. All rights reserved. No reuse allowed without permission.

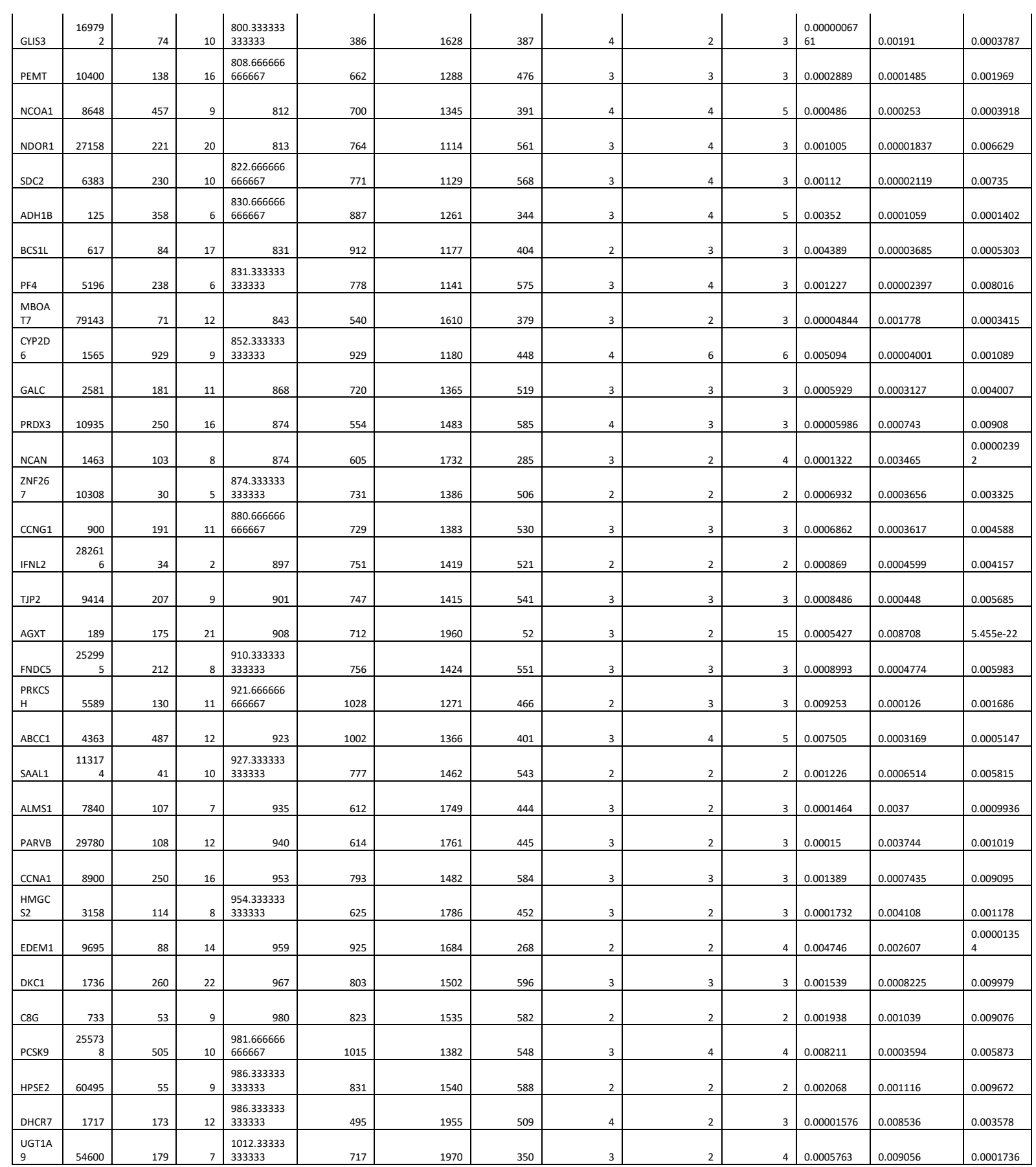


Supplemental Table 2

\begin{tabular}{|c|c|c|c|c|c|c|}
\hline gene.symbol & $\log \mathrm{FC}$ & AveExpr & adj.P.Val & pamrRank & other.score & PSC.score \\
\hline FOXP3 & 2.85210795105714 & 5.55805130552239 & $1.53162271909194 \mathrm{E}-16$ & 1 & -0.2219 & 1.9022 \\
\hline RORC & -1.17587629229047 & 8.56628480147761 & $2.64335277207618 \mathrm{E}-12$ & 5 & 0.1438 & -1.2323 \\
\hline NROB2 & 1.7169309115869 & 9.04411697635075 & $6.11261572747121 \mathrm{E}-11$ & 3 & -0.1582 & 1.3562 \\
\hline $\mathrm{NR} 1 \mathrm{H} 4$ & -0.712794012094047 & 8.8349269801194 & $1.28223642554479 \mathrm{E}-10$ & 17 & 0.1074 & -0.9205 \\
\hline FGF19 & 1.14023062745952 & 3.78925332470149 & $1.55433642960811 \mathrm{E}-10$ & 8 & -0.1332 & 1.142 \\
\hline SLC25A13 & -0.558802832965476 & 10.2026380545746 & $3.34981321586864 \mathrm{E}-10$ & 26 & 0.0925 & -0.7931 \\
\hline THBD & 1.24604327415952 & 6.36105013214179 & $3.79077853892621 \mathrm{E}-10$ & 7 & -0.1352 & 1.1589 \\
\hline SLCO1B1 & -0.780172684142856 & 11.2512259610597 & $3.79077853892621 \mathrm{E}-10$ & 16 & 0.11 & -0.943 \\
\hline TNFRSF1A & 0.661466204839285 & 9.28922832783582 & $3.79077853892621 \mathrm{E}-10$ & 23 & -0.1009 & 0.865 \\
\hline TNFRSF12A & 2.48151812394762 & 7.08810014084328 & $3.79077853892621 \mathrm{E}-10$ & 2 & -0.1667 & 1.4289 \\
\hline LCN2 & 1.45198814049881 & 4.93861709934328 & $9.51260494528471 \mathrm{E}-10$ & 6 & -0.1403 & 1.2022 \\
\hline MMP7 & 1.91032866500952 & 5.11986373857463 & 2.49687091177077E-09 & 4 & -0.1498 & 1.2841 \\
\hline ANXA2 & 1.25734785213333 & 7.56605700483582 & $3.99018536316181 \mathrm{E}-09$ & 10 & -0.1297 & 1.1117 \\
\hline GOT1 & 0.686823386523809 & 11.1131636585821 & $4.55583308289331 \mathrm{E}-09$ & 24 & -0.0994 & 0.8516 \\
\hline Sox9 & 0.793067253722618 & 6.26656025926866 & $2.19527748301191 \mathrm{E}-08$ & 19 & -0.104 & 0.8914 \\
\hline RARA & 0.627855612294047 & 6.89608841800746 & $2.55434616107179 \mathrm{E}-08$ & 27 & -0.0925 & 0.7926 \\
\hline CEBPB & 0.809975239821428 & 9.63041491653731 & 3.53432768007997E-08 & 20 & -0.104 & 0.8913 \\
\hline DGAT2 & -1.15189774165 & 9.57071199290299 & 4.30318075020949E-08 & 12 & 0.1196 & -1.0248 \\
\hline PPARD & 0.750035366583333 & 6.23324932888806 & $6.29204441123426 \mathrm{E}-08$ & 25 & -0.0992 & 0.8507 \\
\hline RORA & 0.790360094770238 & 8.36343632343284 & $6.36224730667225 \mathrm{E}-08$ & 22 & -0.1016 & 0.8705 \\
\hline RELA & 0.503990096264285 & 7.36045555814925 & $1.17940890961085 \mathrm{E}-07$ & 48 & -0.0801 & 0.6866 \\
\hline XBP1 & -0.395305020821428 & 10.9471633776866 & $1.30238701430142 \mathrm{E}-07$ & 73 & 0.0694 & -0.5948 \\
\hline FOXO1 & 0.831022827163094 & 7.89966278085821 & $1.39805484811969 \mathrm{E}-07$ & 21 & -0.1021 & 0.8755 \\
\hline CLDN7 & 0.946410683592857 & 6.77095349418657 & $2.24157250467151 \mathrm{E}-07$ & 18 & -0.1067 & 0.915 \\
\hline PHB & 0.443398718797618 & 9.92754586019403 & $2.65094396654188 \mathrm{E}-07$ & 58 & -0.0735 & 0.6296 \\
\hline MBOAT7 & 0.551692775039286 & 7.28846834633582 & $3.14688617923209 \mathrm{E}-07$ & 42 & -0.0826 & 0.7079 \\
\hline CES1 & -0.351289752178571 & 12.6607570263433 & $3.17595031839151 \mathrm{E}-07$ & 82 & 0.0636 & -0.5452 \\
\hline HNF1B & 0.62901710057619 & 6.47748342310448 & $3.17595031839151 \mathrm{E}-07$ & 31 & -0.0882 & 0.7557 \\
\hline RARRES2 & -0.604635487913095 & 10.3010709824701 & 3.63739732241427E-07 & 35 & 0.0862 & -0.7388 \\
\hline CYP2E1 & -0.439273478297619 & 12.9584501285821 & 4.50228408045603E-07 & 60 & 0.0723 & -0.6197 \\
\hline DLAT & 0.505080317990475 & 7.14178310182836 & 4.92095343667422E-07 & 52 & -0.078 & 0.6686 \\
\hline CTGF & 1.56678735538571 & 7.54055372070895 & $5.41520853822938 \mathrm{E}-07$ & 11 & -0.1232 & 1.0557 \\
\hline MTTP & -0.517609129769048 & 10.4481300806866 & $1.00866274664432 \mathrm{E}-06$ & 53 & 0.078 & -0.6686 \\
\hline ATP11C & -0.639836200705952 & 8.95708644970895 & $1.25233878220911 \mathrm{E}-06$ & 34 & 0.0864 & -0.7402 \\
\hline HMGCS2 & -0.403523366142856 & 12.3225090563433 & $1.99559977782949 \mathrm{E}-06$ & 76 & 0.0671 & -0.5752 \\
\hline KRT7 & 1.36808146231786 & 6.10623529244776 & $2.00699216806864 \mathrm{E}-06$ & 13 & -0.1141 & 0.9778 \\
\hline CDKN1A & 1.33041751169524 & 6.96770206923881 & $2.07152670201502 E-06$ & 14 & -0.1129 & 0.9681 \\
\hline IKBKG & 0.664817888189285 & 5.46399046735821 & $2.21907558079244 \mathrm{E}-06$ & 33 & -0.0867 & 0.7431 \\
\hline CYP7A1 & -2.67253765686309 & 8.30975828408209 & 2.48746689821013E-06 & 9 & 0.1321 & -1.132 \\
\hline DGUOK & 0.491759644992857 & 6.81336796500746 & 2.487466898821013E-06 & 57 & -0.0744 & 0.638 \\
\hline CAV2 & 0.60108008992738 & 8.05232298273881 & 4.00879613719592E-06 & 45 & -0.0815 & 0.6989 \\
\hline ACACA & -0.680500478763095 & 6.94508604124627 & $5.88260482557235 \mathrm{E}-06$ & 36 & 0.0855 & -0.7333 \\
\hline
\end{tabular}




\begin{tabular}{|c|c|c|c|c|c|c|}
\hline LOXL2 & 0.620960266333333 & 5.32455537790299 & $6.25199515796829 \mathrm{E}-06$ & 44 & -0.0819 & 0.7021 \\
\hline ADIPOR2 & -0.453898547328571 & 8.76892005461194 & $8.37226729687356 \mathrm{E}-06$ & 71 & 0.0696 & -0.5962 \\
\hline CD34 & 0.699102314638095 & 6.59317781026866 & 0.0000117138790294528 & 37 & -0.0849 & 0.7281 \\
\hline CEBPA & -0.793585411586904 & 8.71438031098507 & 0.0000117138790294528 & 30 & 0.0895 & -0.7672 \\
\hline MYC & 1.61424268134286 & 6.59621688223881 & 0.0000129018057208315 & 15 & -0.1119 & 0.9589 \\
\hline HMGB1 & -0.380865843883333 & 8.50804924604478 & 0.0000208511773604233 & 86 & 0.0619 & -0.5305 \\
\hline ABCB11 & 0.696177024798809 & 9.13101949159702 & 0.0000212401768152683 & 39 & -0.0834 & 0.7144 \\
\hline $\mathrm{BCHE}$ & -0.878162144288094 & 8.82505379848507 & 0.0000214145947787022 & 28 & 0.0913 & -0.7823 \\
\hline EDN1 & 0.692519889665475 & 5.03712500848507 & 0.0000214145947787022 & 41 & -0.0831 & 0.7119 \\
\hline AKR1D1 & -0.741650069003571 & 10.2809911254702 & 0.0000303580307907452 & 38 & 0.0846 & -0.7249 \\
\hline TNFSF10 & -0.667743597348809 & 11.1823550280522 & 0.0000307854479784407 & 47 & 0.0809 & -0.6934 \\
\hline KRT8 & 0.654853480961904 & 10.5257222183284 & 0.0000359426633022376 & 49 & -0.0798 & 0.6843 \\
\hline FGF23 & 0.841346066421428 & 4.04483400198508 & 0.0000384695044367628 & 32 & -0.088 & 0.7545 \\
\hline FAH & -0.361181739246428 & 10.4072904055821 & 0.0000401514486113887 & 96 & 0.0591 & -0.5062 \\
\hline KLB & -0.627637080961904 & 7.70118748314925 & 0.0000471517350172316 & 54 & 0.0777 & -0.6658 \\
\hline ESR1 & -0.385321687365476 & 5.72605950378358 & 0.0000714244184943412 & 93 & 0.0604 & -0.5178 \\
\hline ADAMTS13 & -0.498702700491666 & 6.52675825677612 & 0.0000717181707249417 & 74 & 0.069 & -0.5914 \\
\hline GLUL & 0.375753703910714 & 9.95733887953731 & 0.0000719134634930595 & 95 & -0.0595 & 0.5102 \\
\hline GPT & 0.668178667007142 & 8.21614935632836 & 0.0000861610714178454 & 51 & -0.0782 & 0.6703 \\
\hline CFLAR & -0.427687659375 & 7.91342681506716 & 0.000102837119225982 & 83 & 0.0632 & -0.5417 \\
\hline ALPL & 0.569195381269047 & 8.6299342456791 & 0.00010304013378728 & 59 & -0.0725 & 0.6216 \\
\hline AGXT & 0.297151385416666 & 11.6572566317164 & 0.000105781433871816 & 122 & -0.0515 & 0.441 \\
\hline $\mathrm{ADH} 1 \mathrm{~B}$ & -0.420335135269047 & 11.6638200793358 & 0.000110152994436119 & 84 & 0.0624 & -0.5352 \\
\hline SMPD1 & 0.358212088783333 & 8.2429873758209 & 0.000113652333728275 & 103 & -0.0572 & 0.4904 \\
\hline GSTP1 & 0.543155824563095 & 7.42688227647015 & 0.000121066775940008 & 67 & -0.0705 & 0.6046 \\
\hline $\mathrm{HP}$ & -0.141445180166667 & 13.4230204505224 & 0.000122277914061661 & 224 & 0.0311 & -0.2662 \\
\hline PTPN1 & 0.322627943541666 & 7.27718730804478 & 0.000138886261048836 & 115 & -0.0536 & 0.4594 \\
\hline GC & -0.159884606273809 & 12.7991564915672 & 0.00014083122763445 & 209 & 0.0339 & -0.2903 \\
\hline HPD & 0.291697116154761 & \begin{tabular}{|l}
12.2249192255224 \\
\end{tabular} & 0.000143360521398941 & 124 & -0.0504 & 0.4323 \\
\hline TAT & 0.54189327895238 & 12.4078977260448 & 0.000167627290450569 & 70 & -0.0696 & 0.5966 \\
\hline $\mathrm{CFH}$ & -0.254308941535714 & 11.6732786213433 & 0.000175716233958485 & 142 & 0.0461 & -0.3953 \\
\hline SOCS1 & 0.565960373196428 & 5.8146556351194 & 0.000175757524775664 & 65 & -0.0708 & 0.6069 \\
\hline SLC22A1 & -0.367494349690476 & 11.8327093847761 & 0.000180175195103295 & 104 & 0.0572 & -0.49 \\
\hline KLF6 & 1.09118751625833 & 7.6004089331194 & 0.000180175195103295 & 29 & -0.0898 & 0.7694 \\
\hline FABP1 & -0.369297595261905 & 12.0579092829851 & 0.000207168264509191 & 105 & 0.0571 & -0.4892 \\
\hline VIM & 0.55934368475357 & 9.08367247161194 & 0.000225651610777606 & 68 & -0.0697 & 0.5979 \\
\hline $\mathrm{ADH} 1 \mathrm{C}$ & -0.469624440559524 & 11.8172474003582 & 0.000228414571329886 & 80 & 0.0643 & -0.5512 \\
\hline LOX & 0.660780969274999 & 4.41239920941045 & 0.000228617913598376 & 56 & -0.0747 & 0.6403 \\
\hline CYP8B1 & 0.302114337555952 & 10.6197026276791 & 0.000229136827457663 & 123 & -0.0508 & 0.4352 \\
\hline KRT18 & 0.600607857790475 & 9.40987241576119 & 0.000277167619946329 & 63 & -0.0713 & 0.611 \\
\hline ANGPT2 & -0.863459607657142 & 4.90330938798507 & 0.000295203370338608 & 46 & 0.0815 & -0.6984 \\
\hline PKD1 & -0.393824832654762 & 7.03502683654478 & 0.000295204972866838 & 99 & 0.0583 & -0.4999 \\
\hline CTNNB1 & -0.281363959194048 & 10.2481889333657 & 0.00032348008610387 & 133 & 0.0482 & -0.4132 \\
\hline C5 & -0.261539200333333 & 11.4438758942537 & 0.000324049273317314 & 143 & 0.0461 & -0.3953 \\
\hline ACACB & -0.445772936514286 & 9.82315152892537 & 0.000376869108113019 & 87 & 0.0615 & -0.5275 \\
\hline
\end{tabular}




\begin{tabular}{|c|c|c|c|c|c|c|}
\hline $\mathrm{NOTCH} 3$ & 0.458883079129762 & 5.96997005670895 & 0.000583931045345888 & 88 & -0.0615 & 0.5268 \\
\hline GLIS3 & 0.451191169496428 & 6.15668248949254 & 0.000600717708196675 & 91 & -0.0609 & 0.5219 \\
\hline CCL13 & 0.224525783110714 & 3.10549420581343 & 0.000925566956958317 & 169 & -0.0408 & 0.3498 \\
\hline CD24 & 1.05736137905714 & 5.67967420664179 & 0.000925566956958317 & 43 & -0.0819 & 0.7022 \\
\hline MMP2 & 0.689846856960714 & 6.24550500654478 & 0.00102984950506741 & 64 & -0.0711 & 0.6094 \\
\hline SDC2 & -0.270308771947619 & 10.3761006362537 & 0.00110120764979831 & 148 & 0.0454 & -0.3894 \\
\hline TMEM30A & -0.290712684916666 & 9.18011395583582 & 0.00110305345197997 & 136 & 0.0474 & -0.4059 \\
\hline JUN & 1.17303463242976 & 8.14793930976119 & 0.00112165942195904 & 40 & -0.0832 & 0.7128 \\
\hline IL6ST & 0.309274613392857 & 10.386394966791 & 0.00112165942195904 & 131 & -0.049 & 0.4198 \\
\hline CD14 & 0.355423840261904 & 11.0379942252239 & 0.00137233955989253 & 118 & -0.0524 & 0.449 \\
\hline COL1A1 & 0.752719768015475 & 7.21903858666418 & 0.00138552343053512 & 61 & -0.0721 & 0.6179 \\
\hline CD5L & -1.01720103627143 & 9.14099316156716 & 0.00147620108454795 & 50 & 0.0787 & -0.6747 \\
\hline LTBP3 & 0.495092561020238 & 8.33054669987313 & 0.00147620108454795 & 89 & -0.0611 & 0.5235 \\
\hline PPARGC1A & 0.710520541098809 & 8.97196702512687 & 0.00179278027859444 & 69 & -0.0697 & 0.5972 \\
\hline PNPLA3 & -0.558192026590476 & 9.11620864879104 & 0.00179290263997411 & 81 & 0.0636 & -0.5453 \\
\hline INSR & 0.271565138574999 & 8.88289361308955 & 0.0018245483702322 & 151 & -0.0447 & 0.3831 \\
\hline HFE & 0.29582425065238 & 6.17992753289552 & 0.00199803740074926 & 138 & -0.0467 & 0.4007 \\
\hline ITGB6 & 0.315041207470238 & 3.07936574197761 & 0.00204741735024337 & 132 & -0.0483 & 0.4141 \\
\hline NQO1 & 0.620282626085714 & 4.60228334020149 & 0.00218518738575201 & 78 & -0.0655 & 0.5617 \\
\hline FOXA2 & 0.403993981267856 & 7.25078328727612 & 0.00247122260364367 & 114 & -0.0543 & 0.4656 \\
\hline TNF & -0.383799183407143 & 4.66795605690298 & 0.00247122260364367 & 116 & 0.053 & -0.4542 \\
\hline IL18 & 0.609623117636904 & 4.28889905980597 & 0.00251975361629049 & 79 & -0.0646 & 0.5533 \\
\hline TIMP1 & 0.676402594288095 & 10.2276795850448 & 0.00252653693756574 & 77 & -0.067 & 0.5741 \\
\hline TJP1 & 0.293762043123809 & 8.68556554947761 & 0.00252653693756574 & 144 & -0.0461 & 0.3948 \\
\hline JAK2 & -0.425884876754762 & 6.24672368229851 & 0.00255144507601513 & 110 & 0.0555 & -0.4757 \\
\hline VEGFA & -0.426501964159524 & 9.28195125231343 & 0.00257485937219156 & 111 & 0.0555 & -0.4756 \\
\hline FGL2 & -0.723646514135714 & 8.00708630266418 & 0.00266280571972731 & 75 & 0.0682 & -0.5848 \\
\hline ICAM1 & 0.843361983080952 & 7.97919057812687 & 0.00278771748253761 & 62 & -0.0714 & 0.612 \\
\hline RXRA & -0.294093300491667 & 9.91201777926866 & 0.00336376406690643 & 147 & 0.0455 & -0.3901 \\
\hline ASS1 & 0.275900212464286 & 12.3274900635821 & 0.00353856675757111 & 155 & -0.0438 & 0.3759 \\
\hline TGFB2 & 0.557219470588094 & 4.65288492359702 & 0.00361843748611693 & 90 & -0.061 & 0.5226 \\
\hline SPP1 & 1.18662012062143 & 7.40273990243284 & 0.00385511488186322 & 55 & -0.0764 & 0.6547 \\
\hline PON1 & -0.280146381809523 & 11.3631959147015 & 0.00398822266767109 & 153 & 0.044 & -0.3769 \\
\hline APOE & 0.246712974892857 & 11.5550814497015 & 0.00409641825016325 & 168 & -0.0409 & 0.3504 \\
\hline PRDX4 & -0.291395728422619 & 8.68729633587313 & 0.0040997672914095 & 149 & 0.0448 & -0.3844 \\
\hline LIPA & -0.494037271129762 & 9.61420247326119 & 0.0044476870927764 & 102 & 0.0574 & -0.4916 \\
\hline LDLR & 0.871278608734523 & 9.62877280828358 & 0.00471167065724233 & 72 & -0.0695 & 0.5954 \\
\hline $\mathrm{CDH} 1$ & -0.257189063504762 & 10.2019006687612 & 0.004841006742395 & 165 & 0.0415 & -0.356 \\
\hline ANGPT1 & 0.360085246053571 & 3.64963814797761 & 0.00495916623493312 & 128 & -0.0495 & 0.4241 \\
\hline MAPK8 & -0.249568587133333 & 7.71696756263433 & 0.00572462069899759 & 171 & 0.0405 & -0.3475 \\
\hline $\mathrm{CTH}$ & 0.484650008519048 & 9.21078201878358 & 0.00615375995611532 & 107 & -0.0558 & 0.4781 \\
\hline ALB & -0.0799170856785724 & 13.3152228429851 & 0.00618422974816703 & 331 & 0.0186 & -0.1598 \\
\hline APOA1 & -0.181764262773809 & 12.691742208806 & 0.00623940243539471 & 211 & 0.0334 & -0.2859 \\
\hline C8G & 0.362946827075 & 9.6169401146791 & 0.00626880806755297 & 130 & -0.049 & 0.42 \\
\hline AHCY & 0.343345867655952 & 9.61579138688806 & 0.00688417529055885 & 134 & -0.0475 & 0.4069 \\
\hline
\end{tabular}




\begin{tabular}{|c|c|c|c|c|c|c|}
\hline FOXA1 & -0.29987517700238 & 8.30017826334328 & 0.00756520968740015 & 152 & 0.0441 & -0.3782 \\
\hline CYP3A7 & -0.585310553890475 & 9.3497110701194 & 0.00756520968740015 & 97 & 0.059 & -0.506 \\
\hline PDCD4 & -0.335110342239285 & 7.31228748423134 & 0.00783271245971037 & 139 & 0.0466 & -0.399 \\
\hline NGF & -0.242930225166666 & 4.66614034687313 & 0.00790099148020295 & 181 & 0.0393 & -0.3366 \\
\hline CYR61 & 1.11199727061428 & 6.98450883791791 & 0.00802204656524097 & 66 & -0.0706 & 0.6054 \\
\hline FGF7 & 0.249809154994048 & 3.36898810324627 & 0.00820346772822923 & 180 & -0.0398 & 0.3411 \\
\hline ATF4 & -0.261592290128571 & 8.19694160442537 & 0.00843982595587419 & 170 & 0.0408 & -0.3493 \\
\hline ERRFI1 & 0.345652490738095 & 11.5810331546269 & 0.00847686520949062 & 137 & -0.047 & 0.4026 \\
\hline SLCO1B3 & -0.572666923472618 & 10.1374389161567 & 0.00882017175216568 & 100 & 0.0578 & -0.4955 \\
\hline LUM & 0.661474916894046 & 7.92533464956716 & 0.00921025519770478 & 92 & -0.0605 & 0.5186 \\
\hline EHHADH & -0.254597385239286 & 10.319606094209 & 0.00921419768125727 & 177 & 0.0399 & -0.3423 \\
\hline STAT4 & -0.547082757755952 & 5.76226403647761 & 0.00929165599931861 & 106 & 0.0566 & -0.4849 \\
\hline SELP & 0.343737988308333 & 5.18001983334328 & 0.00929165599931861 & 141 & -0.0465 & 0.3988 \\
\hline EPHX2 & -0.298900827332142 & 9.21953272132836 & 0.0093950674748333 & 158 & 0.0434 & -0.3719 \\
\hline CDKN2A & 0.302636367315476 & 4.55011508298507 & 0.00942949780846537 & 157 & -0.0436 & 0.374 \\
\hline ACTA2 & 0.394189685825 & 6.12645973420149 & 0.00959295226785569 & 129 & -0.0494 & 0.4234 \\
\hline HMGCS1 & -0.659752871669047 & 9.4010145443806 & 0.0103758468915622 & 94 & 0.0597 & -0.512 \\
\hline PRKCSH & 0.201252520648809 & 10.4791370199627 & 0.0104783744762407 & 204 & -0.0346 & 0.2967 \\
\hline FN1 & -0.135353179319048 & 8.72332516608955 & 0.0104783744762407 & 261 & 0.0268 & -0.2301 \\
\hline VCAM1 & -0.59920756902857 & 7.58338644573134 & 0.0104783744762407 & 101 & 0.0577 & -0.4949 \\
\hline OCLN & 0.464040920819046 & 9.26995547971642 & 0.0106873601683184 & 117 & -0.0524 & 0.4494 \\
\hline STAT6 & 0.219761607486904 & 8.77380466438806 & 0.0107069499815171 & 193 & -0.0364 & 0.3118 \\
\hline $\mathrm{FECH}$ & 0.213801101482143 & 7.00353087088806 & 0.010917837764074 & 195 & -0.0358 & 0.3065 \\
\hline HSPA5 & -0.341240700869048 & 10.9544406180597 & 0.0109847712474079 & 145 & 0.0457 & -0.392 \\
\hline NAMPT & 0.522605490086904 & 10.3935425749328 & 0.0112832382211275 & 113 & -0.0546 & 0.4679 \\
\hline $\mathrm{XDH}$ & -0.242462544878572 & 9.10421601727612 & 0.0114048938496317 & 186 & 0.0383 & -0.3282 \\
\hline SIRT1 & -0.283872815258333 & 6.95072090092537 & 0.0114048938496317 & 164 & 0.0416 & -0.3569 \\
\hline MTHFR & -0.266473411285714 & 6.45999983634328 & 0.0120566722827458 & 175 & 0.0402 & -0.3442 \\
\hline $\mathrm{NR} 1 \mathrm{H} 2$ & 0.209216622476191 & 7.22433489976119 & 0.0124830505084972 & 202 & -0.035 & 0.3003 \\
\hline GYS2 & 0.272765739207142 & 10.3460599562015 & 0.013434858339696 & 174 & -0.0404 & 0.3462 \\
\hline CD69 & 0.579394236849999 & 4.36352608888806 & 0.013806945356894 & 108 & -0.0556 & 0.477 \\
\hline KRT19 & 0.47701967012619 & 4.73806561 & 0.0140553973922545 & 121 & -0.0518 & 0.4438 \\
\hline HAVCR1 & 0.237457976669047 & 2.96358434595522 & 0.015619688540108 & 192 & -0.0372 & 0.3186 \\
\hline TNFAIP3 & 0.496494124146428 & 7.36031487935821 & 0.0163391131722684 & 120 & -0.0519 & 0.4452 \\
\hline SCTR & 0.318267912880952 & 3.98764098482836 & 0.016594402303981 & 160 & -0.043 & 0.3685 \\
\hline FNDC5 & 0.906493419092856 & 7.89100666121642 & 0.0168577647474898 & 85 & -0.0622 & 0.5328 \\
\hline SMAD2 & -0.261492350883333 & 6.83299287102239 & 0.0171120181407762 & 183 & 0.0389 & -0.3333 \\
\hline MAPK14 & -0.19462833652619 & 7.63785294206716 & 0.0173178175229552 & 216 & 0.033 & -0.2825 \\
\hline NPC2 & 0.247167416802381 & 9.4560049034403 & 0.0177761447781849 & 190 & -0.0376 & 0.3226 \\
\hline RPLPO & -0.144646259190476 & 11.381380355597 & 0.0179801647224517 & 254 & 0.0273 & -0.2344 \\
\hline RBP4 & 0.086938295178572 & 13.1588885617164 & 0.0182355384128878 & 324 & -0.0191 & 0.1641 \\
\hline $\mathrm{ABCB} 4$ & 0.374419925466666 & 9.89013820420896 & 0.0190461256172034 & 146 & -0.0457 & 0.3919 \\
\hline TP53 & 0.279512344282143 & 7.50218523481343 & 0.0194122184047791 & 179 & -0.0398 & 0.3415 \\
\hline AGT & -0.161898926785715 & 11.7141416392537 & 0.0201264928129573 & 238 & 0.0292 & -0.2503 \\
\hline FASLG & -0.174626354619048 & 4.1799887553209 & 0.0201264928129573 & 228 & 0.0306 & -0.2623 \\
\hline
\end{tabular}




\begin{tabular}{|c|c|c|c|c|c|c|}
\hline ATP7B & -0.230170105420238 & 7.76245281003731 & 0.0203583393396195 & 194 & 0.0359 & -0.3073 \\
\hline TGFB3 & 0.488930226833333 & 5.81335600967164 & 0.0220896511989663 & 125 & -0.0501 & 0.4297 \\
\hline CD163 & -0.543722463629761 & 7.8060080745 & 0.0221231857209349 & 119 & 0.052 & -0.4457 \\
\hline APOB & -0.0689624926428569 & 12.8945403539552 & 0.0232760258453351 & 355 & 0.0159 & -0.1367 \\
\hline SLC2A2 & -0.172547587678572 & 11.7192592813433 & 0.0250690544867704 & 233 & 0.03 & -0.2571 \\
\hline PDCD1LG2 & -0.504650194617857 & 6.06897231327612 & 0.0264007459308898 & 127 & 0.0498 & -0.4272 \\
\hline DKK1 & 0.206129870557143 & 2.56545417392537 & 0.0271643855849209 & 214 & -0.0331 & 0.2837 \\
\hline HNF1A & 0.263336276907143 & 6.83705366529851 & 0.0273382638371063 & 189 & -0.0377 & 0.3229 \\
\hline TRAPPC11 & -0.170224921713095 & 7.07221086032836 & 0.0285225944230309 & 237 & 0.0295 & -0.2529 \\
\hline SERPINA12 & 0.324817773804762 & 3.64991878808955 & 0.0285225944230309 & 166 & -0.0415 & 0.3554 \\
\hline AHR & -0.300898194492856 & 9.70732151155224 & 0.0299649963205464 & 178 & 0.0399 & -0.3417 \\
\hline TXNIP & 0.395622433838095 & 10.9647146473507 & 0.030928120668662 & 150 & -0.0447 & 0.3834 \\
\hline VWF & 0.438358827172619 & 4.96440029047761 & 0.030928120668662 & 140 & -0.0465 & 0.3989 \\
\hline GPBAR1 & 0.197064517483333 & 4.53188852270149 & 0.0316773231581402 & 221 & -0.0319 & 0.2734 \\
\hline IGFBP1 & 1.00551220627024 & 11.0371807237463 & 0.0332458882200971 & 98 & -0.0585 & 0.5014 \\
\hline CNR1 & 0.141140503525 & 2.77966759973134 & 0.0332458882200971 & 268 & -0.026 & 0.2229 \\
\hline SULT2A1 & 0.285791623584524 & 10.390915422903 & 0.0338583246583664 & 184 & -0.0384 & 0.3296 \\
\hline TNFRSF10A & 0.212698247448809 & 5.79385911536567 & 0.0338583246583664 & 215 & -0.0331 & 0.2836 \\
\hline SERPINB3 & 0.285083344483333 & 2.81977852455224 & 0.0353548934615998 & 188 & -0.0383 & 0.3279 \\
\hline VDR & 0.239937650705952 & 3.95249142076866 & 0.0365550044839184 & 201 & -0.035 & 0.3004 \\
\hline MAPK9 & 0.238878110765476 & 7.59497359064925 & 0.0371203219865998 & 203 & -0.0349 & 0.2993 \\
\hline IL4 & 0.140509170517857 & 2.52856189371642 & 0.0377171430381646 & 270 & -0.0257 & 0.2203 \\
\hline AGER & -0.192084730777381 & 5.13882168102985 & 0.0391211275734287 & 225 & 0.0309 & -0.265 \\
\hline CCND1 & 0.205887967725 & 6.94957280497015 & 0.0393704683270882 & 220 & -0.0321 & 0.2752 \\
\hline BCL2L11 & -0.380125913370238 & 6.25483811727612 & 0.0410315106589983 & 161 & 0.0427 & -0.3659 \\
\hline SERPINE1 & 0.934867324191666 & 9.16031041532089 & 0.0427513524728875 & 109 & -0.0556 & 0.4762 \\
\hline HSP90B1 & -0.146911343809524 & 11.5791771752985 & 0.0427513524728875 & 266 & 0.0262 & -0.2246 \\
\hline HHEX & -0.256133971665476 & 7.77054767061194 & 0.0446200528280309 & 198 & 0.0355 & -0.3047 \\
\hline S100A4 & 0.255227548883333 & 4.47852170792537 & 0.0448831690257445 & 200 & -0.0355 & 0.3039 \\
\hline AREG & 0.530584298516666 & 3.55755005392537 & 0.0457767247395846 & 135 & -0.0474 & 0.4064 \\
\hline CXCL10 & 0.942670117776191 & 7.3499191106194 & 0.0457767247395846 & 112 & -0.055 & 0.4715 \\
\hline TREM1 & 0.42598045447619 & 4.10301807428358 & 0.0462557149635531 & 154 & -0.0439 & 0.3763 \\
\hline AQP1 & 0.41767792925 & 6.90573852835821 & 0.0493400270402151 & 159 & -0.0433 & 0.3709 \\
\hline HES1 & 0.396285186980952 & 7.56781356671642 & 0.0495692202365003 & 162 & -0.0424 & 0.3633 \\
\hline PPARA & -0.189075189498809 & 8.93218809236567 & 0.0495692202365003 & 232 & 0.03 & -0.2573 \\
\hline TNFSF13B & -0.354265093641667 & 5.79875526766418 & 0.0497926208383706 & 172 & 0.0405 & -0.3473 \\
\hline SREBF1 & 0.310045042410714 & 7.636426654 & 0.0500233469597791 & 187 & -0.0383 & 0.328 \\
\hline ARNT & -0.153980971121429 & 9.02850370240298 & 0.0500233469597791 & 262 & 0.0266 & -0.2281 \\
\hline F3 & 0.263545131889285 & 4.68796545684328 & 0.0509584519904039 & 199 & -0.0355 & 0.304 \\
\hline CYP27A1 & 0.206513858914285 & 11.0086666866119 & 0.0525156138472724 & 222 & -0.0313 & 0.2682 \\
\hline DDIT3 & 0.2415082974 & 6.77935393127612 & 0.0525156138472724 & 208 & -0.0339 & 0.2905 \\
\hline IL6 & 0.309971382820238 & 3.19139126145522 & 0.0625745924349403 & 191 & -0.0374 & 0.3202 \\
\hline TTR & -0.0991655703928571 & 12.9259340712687 & 0.0644387421541354 & 319 & 0.0197 & -0.169 \\
\hline CYP4B1 & 0.131179569253571 & 3.09977601930597 & 0.0688462228323515 & 285 & -0.0236 & 0.2019 \\
\hline CXCL9 & 0.816859002615475 & 6.16610278012687 & 0.0690820623568819 & 126 & -0.05 & 0.4286 \\
\hline
\end{tabular}




\begin{tabular}{|c|c|c|c|c|c|c|}
\hline TYMP & 0.260033099615476 & 6.89146193401493 & 0.0697183609568669 & 207 & -0.0341 & 0.2924 \\
\hline PGF & 0.18448740542619 & 4.56498826076866 & 0.0709882872284985 & 242 & -0.0286 & 0.2456 \\
\hline ANXA1 & 0.45081395910119 & 8.16120718641791 & 0.0711786976088793 & 163 & -0.0423 & 0.3624 \\
\hline CCL5 & 0.388904011129762 & 7.09366201279851 & 0.071333679474352 & 176 & -0.0401 & 0.3439 \\
\hline CD40 & 0.167327239195238 & 5.34202885709701 & 0.0717683619684477 & 256 & -0.0271 & 0.2322 \\
\hline ABCB1 & 0.232782000008333 & 7.88897849895522 & 0.0717908691470863 & 219 & -0.0322 & 0.2761 \\
\hline IL1B & -0.511580559315476 & 4.79993909580597 & 0.0730153779543959 & 156 & 0.0437 & -0.3749 \\
\hline EDEM1 & -0.159685752582143 & 6.63149488313433 & 0.0730153779543959 & 264 & 0.0263 & -0.2255 \\
\hline DCLK1 & 0.175224088592857 & 3.0205049033806 & 0.0758716903023264 & 252 & -0.0276 & 0.2367 \\
\hline CXCR3 & 0.18637224645119 & 5.11040392793284 & 0.0769852944184032 & 245 & -0.0285 & 0.2444 \\
\hline ELOVL6 & -0.3749813521 & 8.22396675441045 & 0.0772940992602196 & 182 & 0.0391 & -0.3348 \\
\hline CD274 & 0.357411451998809 & 4.51478436434328 & 0.0780447781274727 & 185 & -0.0383 & 0.3283 \\
\hline ACOX3 & 0.1568888872458332 & 7.62297875277612 & 0.0801207639578954 & 269 & -0.0258 & 0.2211 \\
\hline PTEN & -0.134402819966667 & 9.95681642029851 & 0.0813948841113709 & 286 & 0.0235 & -0.2015 \\
\hline ALMS1 & -0.170677640939285 & 6.21532355338806 & 0.0832657141373954 & 260 & 0.0269 & -0.2309 \\
\hline KEAP1 & 0.151940791645238 & 7.67551786535075 & 0.0853495585717802 & 275 & -0.0252 & 0.2157 \\
\hline BID & 0.194602931997619 & 7.85595109832836 & 0.085620507006878 & 241 & -0.0288 & 0.2468 \\
\hline SERPINA1 & 0.103055869202381 & 11.254724796194 & 0.0856542834332554 & 318 & -0.0198 & 0.1694 \\
\hline COMMD1 & -0.137601224351191 & 7.52704110088806 & 0.0857096780411972 & 283 & 0.0237 & -0.2032 \\
\hline STAT3 & 0.209690018028571 & 10.4353256262313 & 0.0862100400768974 & 235 & -0.0298 & 0.2558 \\
\hline DHCR7 & 0.284562098415476 & 9.36775247943284 & 0.0862100400768974 & 206 & -0.0344 & 0.2946 \\
\hline ADIPOR1 & 0.191472749452381 & 9.68186068494776 & 0.0887989765321462 & 246 & -0.0284 & 0.2434 \\
\hline SHBG & 0.480568337428571 & 7.58958372076866 & 0.0893097148513208 & 167 & -0.0413 & 0.3543 \\
\hline NDOR1 & 0.175072007658333 & 5.2067996506194 & 0.0893097148513208 & 258 & -0.027 & 0.2318 \\
\hline EGF & 0.125533337628571 & 2.86032628663433 & 0.0954374839771327 & 295 & -0.0222 & 0.1901 \\
\hline TIMP2 & 0.271035658210714 & 8.15563282639552 & 0.0969256867023045 & 213 & -0.0331 & 0.2838 \\
\hline YAP1 & 0.161570296246428 & 8.64835466403731 & 0.098221715545508 & 271 & -0.0256 & 0.2194 \\
\hline IDO1 & -0.328622998986904 & 3.87342398989552 & 0.098221715545508 & 196 & 0.0357 & -0.3061 \\
\hline NR3C2 & -0.215097458623809 & 6.78796451295522 & 0.103127885535844 & 236 & 0.0295 & -0.2531 \\
\hline NOS2 & -0.194569433366666 & 3.98110194711194 & 0.105280947316144 & 251 & 0.028 & -0.2402 \\
\hline ANGPTL2 & -0.275274219159524 & 6.72562096047015 & 0.105463194574172 & 217 & 0.0329 & -0.2819 \\
\hline CLU & 0.0693577995119045 & 12.4916406741791 & 0.108304647261217 & 367 & -0.0147 & 0.1263 \\
\hline GPC3 & 0.512841119634524 & 5.31033102949254 & 0.111554245576625 & 173 & -0.0405 & 0.3468 \\
\hline GSK3B & -0.137874177335714 & 7.46675806475373 & 0.114349811811252 & 290 & 0.023 & -0.1971 \\
\hline SAAL1 & -0.150022636154762 & 5.9971179403209 & 0.115080288603776 & 280 & 0.0241 & -0.2067 \\
\hline CAV1 & 0.296165605541666 & \begin{tabular}{|l}
7.88059622416418 \\
\end{tabular} & 0.117933442424765 & 212 & -0.0333 & 0.2853 \\
\hline STK11 & -0.160692945513095 & 6.67618493273881 & 0.11895388626582 & 277 & 0.0249 & -0.2137 \\
\hline MAPK3 & 0.145204441910714 & 7.50368378120895 & 0.125894258987446 & 287 & -0.0234 & 0.2007 \\
\hline TGFB1 & 0.244198324530953 & 6.84547973447015 & 0.126064216101296 & 230 & -0.0304 & 0.2603 \\
\hline IL1R1 & 0.192638200670238 & 8.83740329843284 & 0.127981507661246 & 255 & -0.0271 & 0.2325 \\
\hline CD36 & -0.29121451007619 & 7.75465477335821 & 0.128233148879223 & 218 & 0.0326 & -0.2791 \\
\hline SOD2 & 0.265434811330952 & 9.49430953559702 & 0.129811910314401 & 223 & -0.0313 & 0.2681 \\
\hline CCR5 & -0.131492114296429 & 4.06307426963433 & 0.129811910314401 & 298 & 0.022 & -0.1886 \\
\hline F2R & 0.254890826073809 & 6.995599991059702 & 0.129811910314401 & 227 & -0.0307 & 0.2634 \\
\hline CX3CL1 & 0.247143020402381 & 6.96999823796269 & 0.139958465546539 & 234 & -0.03 & 0.2569 \\
\hline
\end{tabular}




\begin{tabular}{|c|c|c|c|c|c|c|}
\hline CNR2 & -0.117205103555952 & 4.05226015301493 & 0.141374057864564 & 310 & 0.0204 & -0.1745 \\
\hline SLC10A2 & 0.0999517808821426 & 3.18354433735075 & 0.151459113436598 & 335 & -0.0183 & 0.1569 \\
\hline PRF1 & 0.235214958685714 & 5.75763836277612 & 0.151459113436598 & 239 & -0.029 & 0.2481 \\
\hline TNFRSF1B & 0.223333672404762 & 6.97046291642537 & 0.157615693910426 & 250 & -0.0281 & 0.241 \\
\hline ITGAV & 0.227278641249999 & 7.82247797743284 & 0.158810396159408 & 247 & -0.0283 & 0.2424 \\
\hline CLDN1 & 0.139624068309524 & 11.2481469380597 & 0.162008727646207 & 296 & -0.0221 & 0.1897 \\
\hline SREBF2 & -0.179000550334524 & 8.22397731265672 & 0.162360229992693 & 274 & 0.0252 & -0.2159 \\
\hline CASP8 & -0.113665133545238 & 6.37660966890298 & 0.163369092113311 & 320 & 0.0196 & -0.1681 \\
\hline TGFBR1 & 0.119374812477381 & 7.37630218364179 & 0.163369092113311 & 312 & -0.0202 & 0.173 \\
\hline CASP1 & -0.244158751907143 & 5.46939866171642 & 0.163369092113311 & 240 & 0.0289 & -0.2481 \\
\hline HPSE2 & 0.0831263427226189 & 3.14623096026866 & 0.163768573303158 & 354 & -0.0161 & 0.1377 \\
\hline TJP2 & -0.154997404096428 & 8.27195368088806 & 0.166586577460363 & 289 & 0.0233 & -0.1994 \\
\hline CCR2 & -0.213089317566666 & 4.26329921703731 & 0.170418843773816 & 257 & 0.027 & -0.2318 \\
\hline CX3CR1 & -0.211879217922619 & 4.653629488 & 0.170418843773816 & 259 & 0.027 & -0.2312 \\
\hline SLC39A14 & 0.127777945749999 & 10.8066304938806 & 0.17150324119053 & 307 & -0.0208 & 0.1785 \\
\hline FGFR4 & 0.166682351097619 & 8.00470888061194 & 0.17229780466976 & 281 & -0.024 & 0.2057 \\
\hline G6PC & 0.288528979145237 & 11.619212751 & 0.1760980783663 & 229 & -0.0304 & 0.2605 \\
\hline GALC & 0.186023178290476 & 6.6279125495 & 0.176163453750256 & 273 & -0.0252 & 0.2161 \\
\hline TLR9 & -0.13270733847619 & 4.59365414702239 & 0.177155913290023 & 305 & 0.0211 & -0.1812 \\
\hline GPX1 & -0.109928946414286 & 8.44892549989552 & 0.179834451234612 & 327 & 0.0189 & -0.1622 \\
\hline TGFBR2 & 0.171188136304761 & 8.9557288409403 & 0.190231224079201 & 282 & -0.0239 & 0.2048 \\
\hline CBS & 0.128295453085714 & 9.88791037976119 & 0.192781995319782 & 308 & -0.0205 & 0.1756 \\
\hline MCL1 & 0.235314505770238 & 9.53975323677612 & 0.196122627967702 & 253 & -0.0274 & 0.2347 \\
\hline HGF & 0.261932461539286 & 7.53382644174627 & 0.196122627967702 & 244 & -0.0286 & 0.2449 \\
\hline NOTCH1 & -0.1387250327 & 7.15521978606716 & 0.196740918342779 & 303 & 0.0213 & -0.1825 \\
\hline $\mathrm{BCL} 2$ & 0.1286993198 & 5.70751113229105 & 0.198225286389254 & 309 & -0.0204 & 0.1749 \\
\hline FABP4 & -0.449404220294047 & 5.13765175227612 & 0.205702053492177 & 210 & 0.0336 & -0.2879 \\
\hline CCNG1 & 0.185663284211905 & 9.61810877127612 & 0.208352366936575 & 279 & -0.0243 & 0.2087 \\
\hline STK25 & 0.0847667499902381 & 7.35963581698507 & 0.208352366936575 & 358 & -0.0158 & 0.1351 \\
\hline ONECUT2 & 0.170794110372619 & 7.65557836062687 & 0.208949732544786 & 288 & -0.0234 & 0.2003 \\
\hline MMP3 & 0.0909707818202379 & 2.92957249364925 & 0.208949732544786 & 350 & -0.0165 & 0.1411 \\
\hline PKHD1 & -0.276438520351191 & 5.91993493525373 & 0.209693475962963 & 243 & 0.0286 & -0.2452 \\
\hline EGFR & -0.121949304735714 & 8.96002614031343 & 0.211357808808679 & 321 & 0.0195 & -0.1675 \\
\hline IL21 & 0.104335899965476 & 2.5514004650597 & 0.211357808808679 & 338 & -0.0178 & 0.153 \\
\hline HSD11B1 & 0.11619019525119 & 10.0581026695224 & 0.211357808808679 & 325 & -0.019 & 0.1628 \\
\hline CTLA4 & 0.109909789064286 & 3.2164535249403 & 0.211357808808679 & 334 & -0.0184 & 0.1576 \\
\hline SMAD3 & 0.127592652026191 & 7.70377691020149 & 0.211357808808679 & 313 & -0.02 & 0.1714 \\
\hline IGF1 & 0.326322535358333 & 8.12989737785821 & 0.211357808808679 & 231 & -0.0301 & 0.2581 \\
\hline APLN & -0.102836753347619 & 3.74141523772388 & 0.212003053367852 & 341 & 0.0177 & -0.1513 \\
\hline HAVCR2 & -0.237961266532143 & 5.37276937931343 & 0.222582536688757 & 263 & 0.0265 & -0.2274 \\
\hline NR1/2 & -0.166632535425 & 7.66076378105224 & 0.224065770025983 & 293 & 0.0227 & -0.1943 \\
\hline CYP1A2 & -0.28313390180119 & 11.7808542132388 & 0.22496398297865 & 249 & 0.0282 & -0.2418 \\
\hline EGR1 & 0.747589413289285 & 9.59004463903731 & 0.235054695911498 & 197 & -0.0356 & 0.3055 \\
\hline TLR8 & -0.171409614666667 & 4.65925249246269 & 0.242392745217144 & 294 & 0.0226 & -0.1937 \\
\hline UGT1A1 & -0.102489450007143 & 9.01308145466418 & 0.247310905461416 & 342 & 0.0172 & -0.1473 \\
\hline
\end{tabular}




\begin{tabular}{|c|c|c|c|c|c|c|}
\hline REG3A & 0.133467041147619 & 2.79990174729851 & 0.252828291154336 & 316 & -0.0198 & 0.1697 \\
\hline FLT1 & -0.237908255620238 & 6.74477514278358 & 0.258782946612798 & 272 & 0.0255 & -0.2187 \\
\hline AFP & -0.199647517222619 & 4.0060523261791 & 0.262492734490762 & 284 & 0.0237 & -0.2031 \\
\hline IRF1 & -0.26352706665 & 7.64717809604478 & 0.265991714468573 & 265 & 0.0263 & -0.2252 \\
\hline GSTT1 & 0.833820031401191 & 8.61730183191045 & 0.266197466241779 & 205 & -0.0346 & 0.2966 \\
\hline NCAN & 0.102691167865476 & 3.68650149697761 & 0.266197466241779 & 347 & -0.0169 & 0.1452 \\
\hline SPHK1 & 0.184743914127381 & 4.01439626162687 & 0.266197466241779 & 291 & -0.0228 & 0.1953 \\
\hline MYD88 & -0.129802364578572 & 8.52780406707463 & 0.267347156537808 & 323 & 0.0192 & -0.1648 \\
\hline ITPA & -0.1322392035 & 5.50104385831343 & 0.267347156537808 & 322 & 0.0194 & -0.1662 \\
\hline MERTK & 0.160122157289285 & 8.29622230403731 & 0.267347156537808 & 302 & -0.0213 & 0.1826 \\
\hline FGF2 & -0.160881005464286 & 5.83553225599254 & 0.275232217128996 & 304 & 0.0212 & -0.1817 \\
\hline MC4R & 0.105325945832143 & 2.6260966348209 & 0.276202971884456 & 344 & -0.017 & 0.1459 \\
\hline ENTPD2 & 0.0970627483130951 & 4.83897336041045 & 0.277335974668142 & 353 & -0.0162 & 0.1391 \\
\hline CYP3A4 & -0.143023909404762 & 12.4895699993284 & 0.277335974668142 & 314 & 0.02 & -0.1712 \\
\hline LEPR & 0.175409084345237 & 9.60490203632836 & 0.279419075785307 & 300 & -0.0219 & 0.1878 \\
\hline PLIN2 & 0.230382429866667 & 9.84989155723881 & 0.28076292017958 & 278 & -0.0244 & 0.2094 \\
\hline ADIPOQ & -0.0875764880904762 & 2.95894164739552 & 0.280888674273392 & 364 & 0.0152 & -0.1303 \\
\hline LECT2 & -0.182367950138096 & 10.9743902071418 & 0.285472503894445 & 297 & 0.0221 & -0.1895 \\
\hline FAS & 0.161121942872619 & 7.21225851566418 & 0.285472503894445 & 306 & -0.0209 & 0.1794 \\
\hline SAA1 & 0.550468647434524 & 5.7036898824403 & 0.28654786350421 & 226 & -0.0309 & 0.2649 \\
\hline LPIN1 & 0.12958469445 & 7.68710469047015 & 0.28654786350421 & 329 & -0.0188 & 0.1611 \\
\hline AHSG & 0.0701158422738088 & 12.1866152613433 & 0.28654786350421 & 383 & -0.0132 & 0.1127 \\
\hline HMGCR & -0.259092783670237 & 7.98710285193284 & 0.29227731653174 & 276 & 0.0251 & -0.2148 \\
\hline FUT2 & 0.0891472233190473 & 3.56091712447015 & 0.297355842987885 & 365 & -0.0152 & 0.1299 \\
\hline $\mathrm{FOXO3}$ & 0.121306599458333 & 5.87111874286567 & 0.301939775854683 & 337 & -0.0179 & 0.1536 \\
\hline EPCAM & 0.309355458965476 & 4.67690671298507 & 0.301939775854683 & 267 & -0.0262 & 0.2245 \\
\hline CCL25 & 0.0956293862095236 & 4.52379241933582 & 0.302156502235437 & 359 & -0.0157 & 0.1347 \\
\hline HAMP & -0.431387559064286 & 11.0124726403582 & 0.312901085563641 & 248 & 0.0282 & -0.2419 \\
\hline IL13 & 0.105442766545238 & 3.72006392432836 & 0.321083769935639 & 352 & -0.0164 & 0.1403 \\
\hline ABCG8 & 0.154304682946428 & 9.64191138191045 & 0.321083769935639 & 317 & -0.0198 & 0.1695 \\
\hline TERT & 0.0948066564452378 & 4.71526247304478 & 0.333562093631407 & 363 & -0.0153 & 0.1311 \\
\hline PROM1 & 0.159735798022619 & 5.22548954691045 & 0.336653213057345 & 315 & -0.0198 & 0.1697 \\
\hline ADD3 & -0.110090956114286 & 6.95143279720896 & 0.349886768361213 & 351 & 0.0164 & -0.1404 \\
\hline SCD & -0.213330354923809 & 8.37624559452985 & 0.350260348247919 & 299 & 0.0219 & -0.1879 \\
\hline IL2 & 0.0491524252440475 & 2.25011532105224 & 0.356635212917085 & 410 & -0.01 & 0.0854 \\
\hline NFKB1 & 0.100352818610714 & \begin{tabular}{|l}
7.88546330551493 \\
\end{tabular} & 0.357561495512231 & 361 & -0.0155 & 0.1327 \\
\hline ABCG5 & -0.131917433792858 & 8.80987566019403 & 0.357561495512231 & 340 & 0.0178 & -0.1523 \\
\hline MET & 0.120554924526191 & 9.27643885473881 & 0.359046828569645 & 346 & -0.017 & 0.1455 \\
\hline TLR4 & -0.152765451483333 & 7.89133981992537 & 0.359046828569645 & 326 & 0.0189 & -0.1624 \\
\hline SLC10A1 & 0.121528358175 & 11.2909774914701 & 0.359404369356038 & 345 & -0.017 & 0.1459 \\
\hline NLRP3 & -0.118332463083333 & 4.46201101392537 & 0.366809507454208 & 348 & 0.0167 & -0.1431 \\
\hline GLS & -0.150646619875 & 6.02480492828358 & 0.368058643569372 & 330 & 0.0186 & -0.1598 \\
\hline IL1RN & 0.214942461546428 & 7.65023188061194 & 0.373265985796418 & 301 & -0.0213 & 0.1828 \\
\hline OTC & 0.106640382798809 & 10.1604122555896 & 0.37599766726985 & 360 & -0.0157 & 0.1345 \\
\hline S1PR2 & 0.0922209649309522 & 5.74624970197015 & 0.384498990443134 & 371 & -0.0144 & 0.1236 \\
\hline
\end{tabular}




\begin{tabular}{|c|c|c|c|c|c|c|}
\hline IL22 & 0.0543084330476191 & 2.9151741595597 & 0.384498990443134 & 403 & -0.0105 & 0.0896 \\
\hline DMBT1 & 0.0845033703749997 & 3.65100523396269 & 0.384498990443134 & 374 & -0.0137 & 0.1177 \\
\hline IKBKB & 0.0772845568023809 & 6.77484510491791 & 0.387750903199722 & 384 & -0.013 & 0.1115 \\
\hline GNMT & 0.280936135552381 & 9.46524956128358 & 0.38805644249187 & 292 & -0.0227 & 0.1948 \\
\hline LEP & 0.0857087122047618 & 3.5478975659403 & 0.388087330427783 & 373 & -0.0138 & 0.1181 \\
\hline Sox17 & 0.106589959225 & 5.53803113167164 & 0.388087330427783 & 362 & -0.0155 & 0.1326 \\
\hline CXCL12 & -0.111780866516667 & 9.97350896202239 & 0.391568659346461 & 357 & 0.0158 & -0.1353 \\
\hline NCOA1 & -0.080937856961905 & 7.95899148352239 & 0.391568659346461 & 379 & 0.0133 & -0.1138 \\
\hline ENTPD1 & 0.167044863817857 & 6.30885641128358 & 0.395567183599155 & 328 & -0.0188 & 0.1611 \\
\hline CST3 & 0.086385095607143 & 10.8096642607463 & 0.396898572310403 & 375 & -0.0137 & 0.1175 \\
\hline ALOX5 & 0.136468524459524 & 6.09480467422388 & 0.404146706721002 & 343 & -0.0171 & 0.1466 \\
\hline HIF1A & 0.151121192303571 & 8.91758396091045 & 0.409234521540082 & 339 & -0.0178 & 0.1524 \\
\hline CYP2D6 & -0.222738713478571 & 9.3346272346791 & 0.421232392298457 & 311 & 0.0202 & -0.1735 \\
\hline FGA & -0.0388260664404764 & 13.0393234488806 & 0.421232392298457 & 428 & 0.0081 & -0.0695 \\
\hline AGPAT4 & 0.0914595971928573 & 4.24822275075373 & 0.4212348572818 & 372 & -0.0138 & 0.1187 \\
\hline PLAU & 0.100661157939285 & 5.07097384817164 & 0.421472351618369 & 369 & -0.0145 & 0.1246 \\
\hline TGFA & 0.0881095774583333 & 4.85615553478358 & 0.429571470422862 & 377 & -0.0135 & 0.1155 \\
\hline PRDX3 & -0.0569396948214295 & 10.7652216979104 & 0.4348137034449997 & 404 & 0.0104 & -0.0891 \\
\hline ZNF267 & -0.123962586186905 & 5.19406150728358 & 0.434813703444997 & 356 & 0.0158 & -0.1358 \\
\hline DPP4 & -0.13838759357381 & 9.16506212741791 & 0.443646830426147 & 349 & 0.0165 & -0.1413 \\
\hline BMP7 & 0.0561859164559523 & 2.91524484213433 & 0.450352843065234 & 409 & -0.0102 & 0.0874 \\
\hline $\mathrm{ABCC} 3$ & 0.0893495860500001 & 8.86576548082836 & 0.455216714351103 & 380 & -0.0133 & 0.1137 \\
\hline HSD3B7 & -0.089452823669048 & 8.07896378398507 & 0.45562631932361 & 381 & 0.0133 & -0.1136 \\
\hline CFTR & 0.209739445036904 & 4.84167986123881 & 0.475222332250368 & 332 & -0.0185 & 0.1586 \\
\hline CASP3 & -0.070398848836905 & 6.18460045579851 & 0.475222332250368 & 392 & 0.0115 & -0.0982 \\
\hline GGT1 & 0.119194724119047 & 7.36955726029851 & 0.480187377253043 & 366 & -0.0149 & 0.1274 \\
\hline CXCL11 & 0.219457129778571 & 3.87886159199254 & 0.484498693034177 & 333 & -0.0185 & 0.1586 \\
\hline NPC1 & 0.0844065539595235 & 7.42727762437313 & 0.508472523069608 & 387 & -0.0123 & 0.1054 \\
\hline FOXL1 & 0.0616966096261904 & 4.52959340845522 & 0.513533857843241 & 406 & -0.0103 & 0.0884 \\
\hline PCSK9 & 0.102731149509523 & 6.55805648730597 & 0.519277503352977 & 378 & -0.0134 & 0.1146 \\
\hline IFNAR1 & 0.0819329960773809 & 8.49252682097761 & 0.521228106277386 & 389 & -0.012 & 0.1025 \\
\hline HNF4A & -0.0756019204071428 & 10.0572953539478 & 0.522335152762262 & 393 & 0.0114 & -0.0981 \\
\hline ACE & 0.0495026669345237 & 4.66737542913433 & 0.527628561033882 & 423 & -0.0089 & 0.0764 \\
\hline MKI67 & 0.110711937842857 & 3.7686525801791 & 0.534318113564693 & 376 & -0.0136 & 0.1163 \\
\hline JAG1 & 0.131583548903571 & 6.72281229098507 & 0.538201285972226 & 370 & -0.0145 & 0.1243 \\
\hline SOCS3 & 0.272471747870238 & 5.83536710009701 & 0.542687042308827 & 336 & -0.018 & 0.1545 \\
\hline TFF3 & -0.140114686330952 & 5.22446113579104 & 0.544254299683263 & 368 & 0.0147 & -0.1261 \\
\hline BLVRA & 0.0704027081166667 & 5.06301931770149 & 0.548230314759976 & 398 & -0.0107 & 0.0919 \\
\hline TNNI3 & -0.0507539648166667 & 4.34253431177612 & 0.549791778087928 & 424 & 0.0089 & -0.0761 \\
\hline SMN1 & 0.0654094731154761 & 7.15741881079104 & 0.554263601304626 & 408 & -0.0102 & 0.0878 \\
\hline PF4 & 0.0521053760309524 & 4.4045767209403 & 0.563041920148878 & 422 & -0.0089 & 0.0765 \\
\hline NOX4 & 0.0755691315071427 & 3.34589537324627 & 0.565901746571614 & 395 & -0.0109 & 0.0935 \\
\hline ITGB2 & -0.101710839771429 & 7.60105192555224 & 0.565901746571614 & 385 & 0.0125 & -0.1075 \\
\hline NFE2L2 & 0.0881033284630956 & 9.378469887597015 & 0.567580708617001 & 390 & -0.0117 & 0.1005 \\
\hline TM6SF2 & 0.11975204785119 & 8.85938945427612 & 0.578036862533456 & 382 & -0.0132 & 0.1132 \\
\hline
\end{tabular}




\begin{tabular}{|c|c|c|c|c|c|c|}
\hline TIMP3 & 0.0845294905559521 & 7.91147952997015 & 0.592871153250506 & 394 & -0.0112 & 0.0959 \\
\hline MUC16 & 0.0459310639083334 & 3.48160065196269 & 0.59514665295054 & 430 & -0.008 & 0.0689 \\
\hline CD1D & -0.0593038453464285 & 7.17347000563433 & 0.59514665295054 & 416 & 0.0093 & -0.0798 \\
\hline PDGFC & -0.0591987033642859 & 6.96931904749254 & 0.595167555015543 & 417 & 0.0093 & -0.0796 \\
\hline ENG & 0.102510142365476 & 8.99433384136567 & 0.603771060631101 & 388 & -0.012 & 0.1026 \\
\hline C3 & -0.015981004511905 & 13.2201415108955 & 0.605347225383798 & 467 & 0.0038 & -0.0325 \\
\hline MMP1 & 0.0419823769023808 & 3.09025334586567 & 0.607262194143086 & 434 & -0.0075 & 0.0643 \\
\hline $\mathrm{NOTCH} 4$ & 0.0750711177166667 & 5.90999465138806 & 0.611815239002884 & 407 & -0.0103 & 0.088 \\
\hline CPT1A & -0.0974081698059529 & 9.51146226612687 & 0.611815239002884 & 391 & 0.0115 & -0.0989 \\
\hline VPS33B & -0.0450952141059523 & 5.68389323711194 & 0.611815239002884 & 431 & 0.0078 & -0.0667 \\
\hline ITGB4 & 0.0687910846047617 & 4.63095052520895 & 0.6143795983118 & 413 & -0.0098 & 0.0839 \\
\hline CALCA & -0.0577232170547619 & 3.69455450311194 & 0.623619067148061 & 425 & 0.0088 & -0.0759 \\
\hline BCS1L & -0.0466547899011903 & 6.71429301072388 & 0.635657416404078 & 432 & 0.0078 & -0.0666 \\
\hline PROX1 & 0.0619064193357135 & 9.23217045380597 & 0.641123370656377 & 421 & -0.009 & 0.0772 \\
\hline POSTN & 0.132752088996428 & 5.84154263981343 & 0.64406690597194 & 386 & -0.0123 & 0.1056 \\
\hline HPX & 0.0303359404404764 & 12.5727448940299 & 0.644291067986128 & 450 & -0.0059 & 0.0504 \\
\hline NR113 & -0.0788091418630955 & 8.98290843135075 & 0.652489881810128 & 412 & 0.0099 & -0.0852 \\
\hline PARVB & -0.0601179031107143 & 7.07163276237313 & 0.658703961796268 & 427 & 0.0087 & -0.0742 \\
\hline $\mathrm{ABCC} 4$ & 0.0949384094202377 & 4.96712460280597 & 0.658703961796268 & 401 & -0.0106 & 0.0911 \\
\hline MIF & 0.106224119380953 & 9.48657328065672 & 0.670704121720404 & 396 & -0.0109 & 0.0931 \\
\hline F5 & -0.0412009969666672 & 10.656998543194 & 0.670704121720404 & 436 & 0.0069 & -0.0594 \\
\hline IL12B & 0.0350490620785714 & 3.16368775115672 & 0.670704121720404 & 448 & -0.0063 & 0.0539 \\
\hline MAT1A & -0.0403209954523811 & 11.8518390998507 & 0.676246346074837 & 439 & 0.0068 & -0.0583 \\
\hline ARG1 & 0.0492382114607145 & 10.4552084235746 & 0.684515667080014 & 433 & -0.0075 & 0.0644 \\
\hline CPS1 & 0.0324284685476188 & 12.3506472061194 & 0.684515667080014 & 449 & -0.0059 & 0.0504 \\
\hline $\mathrm{CP}$ & 0.0272446989523811 & 12.5199615414179 & 0.684515667080014 & 455 & -0.0053 & 0.045 \\
\hline TLR3 & -0.0785155262476192 & 8.28718751685075 & 0.684515667080014 & 415 & 0.0093 & -0.0801 \\
\hline PDGFRB & -0.0730218898642853 & 7.33472198947761 & 0.684515667080014 & 420 & 0.0091 & -0.0776 \\
\hline MACC1 & -0.0769609451761905 & 3.76545721026866 & 0.691602152564866 & 419 & 0.0092 & -0.0785 \\
\hline MTOR & 0.0437373633714286 & 8.07918388847761 & 0.696138231619637 & 438 & -0.0069 & 0.0592 \\
\hline FGF21 & -0.126446297010714 & 5.38644866307463 & 0.696138231619637 & 397 & 0.0108 & -0.0928 \\
\hline CXCR2 & 0.100897255677381 & 3.59675890643284 & 0.70125571725802 & 411 & -0.01 & 0.0853 \\
\hline IL33 & 0.0862045754333333 & 5.93547307774627 & 0.708065667747015 & 418 & -0.0093 & 0.0796 \\
\hline UCP2 & 0.0939344193428567 & 7.22064413361194 & 0.710769481142648 & 414 & -0.0095 & 0.0816 \\
\hline GOLM1 & 0.1277030018 & 7.28275777936567 & 0.719580125961173 & 405 & -0.0103 & 0.0885 \\
\hline IFNG & -0.0454501675392858 & 2.93933436135821 & 0.726552366397051 & 442 & 0.0067 & -0.0578 \\
\hline PSMD10 & 0.044296729377381 & 7.72669076241045 & 0.728396408512551 & 443 & -0.0066 & 0.0568 \\
\hline ATP8B1 & -0.0313892402666666 & 6.26486223090298 & 0.728396408512551 & 453 & 0.0054 & -0.0467 \\
\hline CRP & -0.168345280091667 & 10.7246069782463 & 0.732071662198825 & 400 & 0.0107 & -0.0917 \\
\hline CXCL13 & -0.172342391705952 & 3.78729386747015 & 0.738313778877388 & 402 & 0.0106 & -0.0907 \\
\hline CHI3L1 & 0.198500750617858 & 7.95947890741791 & 0.744073837965965 & 399 & -0.0107 & 0.0919 \\
\hline PCK1 & 0.0732408992297616 & 11.8772930785896 & 0.745124619372517 & 429 & -0.008 & 0.0689 \\
\hline TLR7 & -0.0519510354178573 & 3.78061851865672 & 0.760342132965275 & 440 & 0.0067 & -0.0578 \\
\hline DLK1 & 0.0570278788869047 & 4.67596630064179 & 0.760342132965275 & 435 & -0.007 & 0.0603 \\
\hline F2 & 0.0266572272976187 & 11.7016734918657 & 0.760342132965275 & 460 & -0.0047 & 0.0404 \\
\hline
\end{tabular}




\begin{tabular}{|c|c|c|c|c|c|c|}
\hline HBEGF & 0.120511047189285 & 5.93074080894776 & 0.773122901700071 & 426 & -0.0088 & 0.0757 \\
\hline APLNR & -0.0514710949142852 & 7.4508507861194 & 0.781012122472672 & 445 & 0.0064 & -0.055 \\
\hline IL2RA & 0.0579382513095237 & 4.70300065897015 & 0.781012122472672 & 441 & -0.0067 & 0.0578 \\
\hline GPX4 & -0.0281333474999994 & 11.3135517075373 & 0.781012122472672 & 461 & 0.0047 & -0.0403 \\
\hline LBP & 0.0383088840595237 & 11.648307168806 & 0.782854689934759 & 452 & -0.0055 & 0.0475 \\
\hline LGALS3 & -0.0523509998511903 & 5.53007165526119 & 0.782854689934759 & 446 & 0.0064 & -0.0548 \\
\hline STAT1 & 0.0551642287833332 & 9.304399566110448 & 0.790732545259505 & 444 & -0.0064 & 0.0551 \\
\hline SLC27A5 & 0.0389944527488097 & 10.1986881579104 & 0.803094215490467 & 454 & -0.0054 & 0.046 \\
\hline MST1 & -0.0344035276154769 & 9.91003401335075 & 0.805526028537823 & 458 & 0.005 & -0.0429 \\
\hline MPO & 0.0378422344226189 & 3.71581862657463 & 0.812671550517039 & 456 & -0.0052 & 0.0442 \\
\hline IL17A & -0.0220524269964287 & 3.95805772851493 & 0.812671550517039 & 466 & 0.0038 & -0.0328 \\
\hline CXCL2 & -0.0820651115154758 & 8.45482374378358 & 0.812671550517039 & 437 & 0.0069 & -0.0593 \\
\hline MBL2 & -0.0641683111749999 & 10.7078374602612 & 0.813138873737447 & 447 & 0.0064 & -0.0544 \\
\hline ACE2 & -0.0518047316059521 & 5.63714470824627 & 0.817866191049633 & 451 & 0.0058 & -0.0495 \\
\hline NR5A2 & 0.0258405146892853 & 8.94291250079104 & 0.833866297149495 & 465 & -0.004 & 0.0344 \\
\hline CXCL16 & 0.0424238138833334 & 7.2954830328806 & 0.833866297149495 & 457 & -0.0051 & 0.0437 \\
\hline ONECUT1 & -0.0306621572535714 & 9.41820636785821 & 0.842423626623655 & 463 & 0.0043 & -0.0368 \\
\hline EZR & 0.0220529710809524 & 7.66777120388806 & 0.853461987704616 & 470 & -0.0035 & 0.0302 \\
\hline $\mathrm{NOTCH} 2$ & 0.0164086490607144 & 8.84303240619403 & 0.860781670501423 & 477 & -0.0029 & 0.0249 \\
\hline PTPRO & 0.0194915775642856 & 3.88685132762687 & 0.870546941224976 & 474 & -0.0031 & 0.027 \\
\hline CXCL3 & 0.0241803401166664 & 4.86635372457463 & 0.870546941224976 & 469 & -0.0035 & 0.0302 \\
\hline TLR2 & 0.0385108976988094 & 5.40718882278358 & 0.870546941224976 & 462 & -0.0043 & 0.0371 \\
\hline NPPB & -0.0174240505428569 & 3.61812246620896 & 0.871022564058828 & 476 & 0.0029 & -0.025 \\
\hline BHMT & -0.024637113672619 & 11.8656515911567 & 0.878429860460012 & 472 & 0.0034 & -0.0294 \\
\hline LYZ & 0.0622068991047623 & 9.20094891223134 & 0.878429860460012 & 459 & -0.0049 & 0.0417 \\
\hline TNFAIP8L2 & -0.0182591335095236 & 4.83456712370149 & 0.888881577391701 & 478 & 0.0028 & -0.0243 \\
\hline HMOX1 & 0.0432795220190472 & 8.24304956637313 & 0.890223932108496 & 464 & -0.0041 & 0.0352 \\
\hline NCF1 & 0.0230657531785714 & 5.8379397805597 & 0.896614580372748 & 475 & -0.0031 & 0.0265 \\
\hline NR1H3 & -0.0138399287619049 & 7.15686303062687 & 0.90374031469662 & 481 & 0.0023 & -0.0198 \\
\hline CYP7B1 & -0.0169289319571433 & 8.37005425121642 & 0.914600977757212 & 480 & 0.0025 & -0.0212 \\
\hline NOX1 & -0.00896245997619049 & 3.18018607141791 & 0.914600977757212 & 490 & 0.0017 & -0.0144 \\
\hline CD44 & -0.0318211268511902 & 8.38638876986567 & 0.914600977757212 & 473 & 0.0032 & -0.027 \\
\hline GFER & 0.0133962262392853 & 5.51469317670149 & 0.914600977757212 & 484 & -0.0021 & 0.0183 \\
\hline CCL2 & 0.0542809860059522 & 7.7686881395 & 0.914600977757212 & 468 & -0.0036 & 0.0312 \\
\hline PTGS2 & -0.0454644690511903 & 5.49191296875373 & 0.914600977757212 & 471 & 0.0035 & -0.0297 \\
\hline PARP1 & 0.0151623832940476 & 7.74480460758955 & 0.914600977757212 & 482 & -0.0022 & 0.0192 \\
\hline SLC40A1 & -0.0151401280464287 & 10.3941305725522 & 0.914600977757212 & 483 & 0.0022 & -0.0191 \\
\hline SMAD7 & -0.012443632607143 & 6.13823056323134 & 0.927063725088991 & 486 & 0.0019 & -0.0163 \\
\hline PDGFB & -0.0123636447619047 & 4.58011073725373 & 0.927063725088991 & 487 & 0.0019 & -0.0162 \\
\hline MMP9 & -0.0327138597226191 & 4.59747275806716 & 0.927063725088991 & 479 & 0.0028 & -0.024 \\
\hline NOS3 & -0.0127774251309522 & 5.56593831605224 & 0.933191773623727 & 488 & 0.0018 & -0.0156 \\
\hline PPARG & -0.0126946449297621 & 7.12303202167164 & 0.933191773623727 & 489 & 0.0018 & -0.0156 \\
\hline EPO & 0.0173009869654761 & 5.22336722769403 & 0.933742644472953 & 485 & -0.0021 & 0.0177 \\
\hline PDCD1 & 0.00992990592142844 & 4.29050097750746 & 0.937468992301964 & 494 & -0.0015 & 0.0132 \\
\hline CDK1 & 0.0106335902773809 & 2.68010164712687 & 0.938594809746976 & 492 & -0.0016 & 0.0134 \\
\hline
\end{tabular}


bioRxiv preprint doi: https://doi.org/10.1101/2021.09.06.459063; this version posted September 6, 2021. The copyright holder for this preprint (which was not certified by peer review) is the author/funder. All rights reserved. No reuse allowed without permission.

\begin{tabular}{|l|c|c|c|c|c|c|} 
AGTR1 & -0.0124548317547619 & 8.31198545910448 & 0.94736599397164 & 491 & 0.0016 & -0.0136 \\
\hline IL10 & -0.0132236207738094 & 4.15915672359701 & 0.951217304177938 & 493 & 0.0016 & -0.0134 \\
\hline PEMT & 0.00863647137499999 & 11.0084380715299 & 0.952476659299077 & 496 & -0.0013 & 0.0107 \\
\hline IL23A & -0.00416362960714275 & 4.12666963317164 & 0.952476659299077 & 498 & 0.0008 & -0.0069 \\
\hline ENPP2 & 0.0182474916059518 & 8.02844441945522 & 0.965743200631755 & 495 & -0.0014 & 0.0119 \\
\hline ABCC1 & 0.00694043438928573 & 6.06111009633582 & 0.965743200631755 & 497 & -0.001 & 0.0082 \\
\hline CD40LG & 0.00456193552499985 & 3.20529193410448 & 0.979077344016075 & 501 & -0.0006 & 0.0055 \\
\hline MX1 & -0.00604163464761933 & 6.53515132287313 & 0.979077344016075 & 500 & 0.0007 & -0.006 \\
\hline PLAUR & 0.009011871655595247 & 5.633326500888806 & 0.979077344016075 & 499 & -0.0008 & 0.0066 \\
\hline EDNRA & -0.00533701776547612 & 6.46687138903731 & 0.979077344016075 & 502 & 0.0006 & -0.0054 \\
\hline RETN & -0.00267083347738083 & 4.20917191438806 & 0.979077344016075 & 503 & 0.0004 & -0.0038 \\
\hline SLC4A2 & -0.00230366552380932 & 7.46496963219403 & 0.980741196117166 & 504 & 0.0004 & -0.0032 \\
\hline CCNA1 & 0.00169430916190471 & 3.10310191264179 & 0.980741196117166 & 506 & -0.0003 & 0.0026 \\
\hline DKC1 & 0.002319289333809521 & 6.294365166544478 & 0.980741196117166 & 505 & -0.0004 & 0.003 \\
\hline ABCC2 & 0.00107757839404723 & 10.256582001709 & 0.988819475802519 & 507 & -0.0002 & 0.0015 \\
\hline
\end{tabular}


Supplemental table 3

\begin{tabular}{|c|c|c|}
\hline gene symbol & PTM.R.values & PTM.p.Values \\
\hline TNF & 0.658481 & 0.019895649 \\
\hline NR1H4 & 0.8987596 & 7.051707E-5 \\
\hline TNFRSF1A & 0.606782 & 0.036433317 \\
\hline MIF & -0.8562979 & 3.7723436E-4 \\
\hline IFNG & -0.62242836 & 0.03065831 \\
\hline ABCB11 & 0.85081524 & $4.5052322 \mathrm{E}-4$ \\
\hline IL1RN & 0.608279 & 0.035849635 \\
\hline IL1R1 & 0.582369 & 0.04693469 \\
\hline LDLR & 0.6105657 & 0.03497096 \\
\hline CYP7A1 & -0.64633787 & 0.023148783 \\
\hline TLR3 & 0.8164091 & 0.0011961266 \\
\hline STAT1 & 0.6501415 & 0.022091046 \\
\hline CD274 & 0.69797415 & 0.011598374 \\
\hline NR112 & 0.746237 & 0.0053126863 \\
\hline KRT18 & 0.8542353 & 4.0362534E-4 \\
\hline HIF1A & -0.57752514 & 0.049247243 \\
\hline C3 & 0.7234944 & 0.007823705 \\
\hline CoL1A1 & -0.6196485 & 0.03163258 \\
\hline CTNNB1 & -0.57828486 & 0.04887935 \\
\hline MET & 0.70211357 & 0.010909507 \\
\hline ANXA1 & -0.58730876 & 0.044656325 \\
\hline NROB2 & 0.5881663 & 0.044268925 \\
\hline CD36 & 0.83506334 & $7.237027 \mathrm{E}-4$ \\
\hline PTEN & 0.65347886 & 0.021192333 \\
\hline KRT8 & 0.82332855 & 9.993713E-4 \\
\hline PPARGC1A & 0.8274073 & $8.957106 \mathrm{E}-4$ \\
\hline TIMP3 & -0.8154762 & 0.00122478 \\
\hline PKHD1 & 0.66367006 & 0.018612774 \\
\hline CASP8 & -0.5922592 & 0.04245258 \\
\hline CEBPA & -0.8422907 & 5.859107E-4 \\
\hline IGF1 & 0.58007735 & 0.048019014 \\
\hline NOX4 & 0.69428235 & 0.012239512 \\
\hline SLC10A2 & 0.80886513 & 0.0014432635 \\
\hline SPHK1 & -0.6650197 & 0.018289283 \\
\hline KLF6 & 0.5804634 & 0.047835127 \\
\hline IRF1 & 0.83695173 & $6.8550755 \mathrm{E}-4$ \\
\hline ADIPOR2 & -0.72480935 & 0.007658131 \\
\hline TGFBR1 & -0.8153795 & 0.0012277792 \\
\hline TIMP2 & -0.7353816 & 0.0064204982 \\
\hline GOLM1 & 0.717523 & 0.008609553 \\
\hline ENPP2 & 0.7837536 & 0.0025568428 \\
\hline SLC4A2 & -0.7578264 & 0.0042959247 \\
\hline
\end{tabular}




\begin{tabular}{|c|c|c|}
\hline RARRES2 & 0.8541692 & 4.0449417E-4 \\
\hline FABP1 & -0.68153894 & 0.014655506 \\
\hline PDGFRB & -0.84134036 & $6.0276163 \mathrm{E}-4$ \\
\hline CYP1A2 & -0.6274603 & 0.028949907 \\
\hline GPT & 0.85449815 & 4.0018465E-4 \\
\hline BCL2L11 & -0.85782444 & 3.5858207E-4 \\
\hline NQO1 & 0.891083 & $1.002805 \mathrm{E}-4$ \\
\hline MCL1 & 0.65469617 & 0.020871263 \\
\hline TERT & 0.78323156 & 0.0025854032 \\
\hline AQP1 & -0.6413803 & 0.024582073 \\
\hline CLU & 0.6294113 & 0.028306369 \\
\hline MST1 & 0.71179026 & 0.009418075 \\
\hline CXCL11 & 0.8591084 & $3.4345628 \mathrm{E}-4$ \\
\hline GSTP1 & -0.69853413 & 0.011503345 \\
\hline VIM & -0.7176681 & 0.008589781 \\
\hline MERTK & -0.6456616 & 0.023340631 \\
\hline BLVRA & 0.5798412 & 0.048131745 \\
\hline NPC1 & -0.7495386 & 0.005006292 \\
\hline MTTP & -0.66207 & 0.019001702 \\
\hline SREBF2 & 0.6123078 & 0.034311987 \\
\hline CTH & 0.6153943 & 0.033166353 \\
\hline FABP4 & -0.75353557 & 0.0046534413 \\
\hline MAT1A & 0.69206965 & 0.012636151 \\
\hline $\mathrm{HPX}$ & 0.70675 & 0.01017455 \\
\hline MX1 & -0.59253365 & 0.042332724 \\
\hline CST3 & 0.7293835 & 0.0071025416 \\
\hline PSMD10 & 0.73154086 & 0.0068512647 \\
\hline LPIN1 & -0.6256573 & 0.02955394 \\
\hline G6PC & -0.6595853 & 0.019617384 \\
\hline DGAT2 & -0.6934754 & 0.012383074 \\
\hline SLC27A5 & 0.6131261 & 0.034005534 \\
\hline CYP8B1 & -0.66253716 & 0.018887538 \\
\hline AKR1D1 & 0.7719539 & 0.0032640218 \\
\hline GGT1 & 0.7714968 & 0.0032941196 \\
\hline HMGCS1 & 0.62166053 & 0.030925227 \\
\hline LUM & -0.6428049 & 0.024163788 \\
\hline TTR & -0.66472155 & 0.018360388 \\
\hline GSTT1 & -0.6366131 & 0.026020017 \\
\hline $\mathrm{BCHE}$ & 0.7080673 & 0.0099726245 \\
\hline PEMT & 0.85449916 & 4.001714E-4 \\
\hline DCLK1 & 0.6461042 & 0.023214936 \\
\hline NCOA1 & -0.5952546 & 0.04115723 \\
\hline GALC & -0.8746848 & $1.9648828 \mathrm{E}-4$ \\
\hline ALPL & -0.6931997 & 0.012432414 \\
\hline CCNA1 & -0.5844916 & 0.04594588 \\
\hline
\end{tabular}


bioRxiv preprint doi: https://doi.org/10.1101/2021.09.06.459063; this version posted September 6, 2021. The copyright holder for this preprint (which was not certified by peer review) is the author/funder. All rights reserved. No reuse allowed without permission.

\begin{tabular}{|l|l|l|} 
PRDX3 & -0.8598483 & $3.349677 \mathrm{E}-4$ \\
\hline C8G & 0.7698487 & 0.0034043903 \\
\hline MBOAT7 & 0.6840867 & 0.014146564 \\
\hline EDEM1 & 0.68641347 & 0.013693266 \\
\hline
\end{tabular}


bioRxiv preprint doi: https://doi.org/10.1101/2021.09.06.459063; this version posted September 6, 2021. The copyright holder for this preprint (which was not certified by peer review) is the author/funder. All rights reserved. No reuse allowed without permission.

Supplemental Table 4

\begin{tabular}{|c|c|c|c|c|}
\hline gene symbol & num_cells_expressed & proportion positive & P-values & Q-values \\
\hline Sox9 & 1027 & 78.0395136778116 & $7.19816577256051 \mathrm{E}-246$ & $8.86526096548553 \mathrm{E}-242$ \\
\hline NrOb2 & 457 & 34.726443768997 & 7.80162701157387E-243 & 4.80424191372719E-239 \\
\hline Malat1 & 1310 & 99.5440729483283 & $1.76035519723752 \mathrm{E}-071$ & $2.16805346091773 \mathrm{E}-068$ \\
\hline Spp1 & 1303 & 99.0121580547113 & $5.39371002427612 \mathrm{E}-033$ & $5.53574438824873 \mathrm{E}-030$ \\
\hline Mgst1 & 1130 & 85.8662613981763 & $1.47597176822542 \mathrm{E}-031$ & $1.39831294595879 \mathrm{E}-028$ \\
\hline Tstd1 & 1014 & 77.0516717325228 & $2.3504283690298 \mathrm{E}-029$ & $2.06770541378365 \mathrm{E}-026$ \\
\hline Eef1a1 & 1229 & 93.3890577507599 & $1.22468548648441 \mathrm{E}-028$ & $1.0055484301028 \mathrm{E}-025$ \\
\hline Pebp1 & 1144 & 86.9300911854103 & 4.02906802976077E-027 & $3.10137511590835 \mathrm{E}-024$ \\
\hline Fth1 & 1239 & 94.1489361702128 & $1.59753258665412 \mathrm{E}-026$ & $1.09306729651289 \mathrm{E}-023$ \\
\hline Bsg & 1171 & 88.9817629179331 & $1.88253916849066 \mathrm{E}-026$ & $1.22028170521742 \mathrm{E}-023$ \\
\hline Ubb & 1219 & 92.629179331307 & $8.97572503677164 \mathrm{E}-026$ & $5.52725147764397 \mathrm{E}-023$ \\
\hline Gm42418 & 1299 & 98.7082066869301 & $2.40003585125472 \mathrm{E}-025$ & $1.40756388305015 \mathrm{E}-022$ \\
\hline Ttr & 1198 & 91.0334346504559 & 4.63013967737829E-025 & $2.59203637575414 \mathrm{E}-022$ \\
\hline Sorbs2 & 1099 & 83.5106382978723 & $6.2231374271315 \mathrm{E}-025$ & $3.33235480663268 \mathrm{E}-022$ \\
\hline Mpc2 & 943 & 71.6565349544073 & $1.78839080918745 \mathrm{E}-023$ & $8.81032848238105 \mathrm{E}-021$ \\
\hline Tpt1 & 1211 & 92.0212765957447 & $2.76716898382304 \mathrm{E}-023$ & $1.31078666172172 \mathrm{E}-020$ \\
\hline Birc2 & 640 & 48.6322188449848 & $5.18588759305987 \mathrm{E}-023$ & $2.28104969986162 \mathrm{E}-020$ \\
\hline Dbi & 1188 & 90.273556231003 & $8.89634465670118 \mathrm{E}-022$ & 3.49103392867551E-019 \\
\hline Eno1 & 1013 & 76.9756838905775 & $9.07056558278793 \mathrm{E}-022$ & $3.49103392867551 \mathrm{E}-019$ \\
\hline Fau & 1193 & 90.6534954407295 & $2.2382807335081 \mathrm{E}-021$ & $8.35353500420782 \mathrm{E}-019$ \\
\hline $\mathrm{C} 3$ & 1165 & 88.5258358662614 & $5.89060939166066 \mathrm{E}-021$ & $1.96077689912683 \mathrm{E}-018$ \\
\hline Anxa5 & 1241 & 94.3009118541034 & 1.17336272031784E-020 & 3.80293033248275E-018 \\
\hline Atp5g2 & 1060 & 80.5471124620061 & $3.55598889703684 \mathrm{E}-020$ & $1.06818437209526 \mathrm{E}-017$ \\
\hline Tmem45a & 1092 & 82.9787234042553 & $9.19970538855949 \mathrm{E}-020$ & $2.63496678059299 \mathrm{E}-017$ \\
\hline Tmem176b & 1243 & 94.4528875379939 & $1.30116534563943 \mathrm{E}-019$ & 3.56114497708782E-017 \\
\hline Agrn & 422 & 32.0668693009119 & 5.40861819889119E-019 & $1.4172881220754 \mathrm{E}-016$ \\
\hline Apoc1 & 979 & 74.3920972644377 & $7.18057911761755 \mathrm{E}-019$ & $1.80481657984853 \mathrm{E}-016$ \\
\hline Ppia & 1199 & 91.1094224924012 & $2.38690303927379 \mathrm{E}-018$ & $5.65328804455693 \mathrm{E}-016$ \\
\hline Cox4i1 & 1157 & 87.9179331306991 & $3.66700241608377 \mathrm{E}-018$ & $8.21141850117958 \mathrm{E}-016$ \\
\hline Mrpl14 & 693 & 52.6595744680851 & $3.65807587824824 \mathrm{E}-018$ & $8.21141850117958 \mathrm{E}-016$ \\
\hline Tmem59 & 1078 & 81.9148936170213 & $6.07997019984648 \mathrm{E}-018$ & $1.33715916038052 \mathrm{E}-015$ \\
\hline Dad1 & 955 & 72.5683890577508 & $1.22868758853049 \mathrm{E}-017$ & $2.60905454143819 \mathrm{E}-015$ \\
\hline Oaz1 & 1118 & 84.9544072948328 & $1.66390966513063 \mathrm{E}-017$ & 3.47334092131336E-015 \\
\hline Ndufa4 & 1117 & 84.8784194528875 & $2.3471410683842 \mathrm{E}-017$ & $4.81789823303664 \mathrm{E}-015$ \\
\hline Ndfip1 & 938 & 71.2765957446809 & $3.04424805295822 \mathrm{E}-017$ & $6.04725145487637 \mathrm{E}-015$ \\
\hline Gstm1 & 1068 & 81.1550151975684 & $3.99563738498861 \mathrm{E}-017$ & 7.68910469273745E-015 \\
\hline Tle5 & 1086 & 82.5227963525836 & $4.11244014866789 \mathrm{E}-017$ & 7.79212505707595E-015 \\
\hline Kcnq1 ot1 & 444 & 33.7386018237082 & $1.34139849502433 \mathrm{E}-016$ & 0.0000000000000241456125018703 \\
\hline Gsta3 & 763 & 57.9787234042553 & $1.56486849650494 \mathrm{E}-016$ & 0.0000000000000275327434327927 \\
\hline Id2 & 1079 & 81.9908814589666 & $1.60027828590538 \mathrm{E}-016$ & 0.0000000000000277591934777614 \\
\hline Col18a1 & 525 & 39.8936170212766 & $2.38652233320723 \mathrm{E}-016$ & 0.000000000000040263574049014 \\
\hline Clu & 1308 & 99.3920972644377 & $2.386246313162 \mathrm{E}-016$ & 0.000000000000040263574049014 \\
\hline Onecut2 & 1043 & 79.2553191489362 & $2.62852289518562 \mathrm{E}-016$ & 0.0000000000000437471459150083 \\
\hline Afdn & 775 & 58.8905775075988 & $3.10321434495412 \mathrm{E}-016$ & 0.0000000000000509589171632732 \\
\hline Btf3 & 1064 & 80.8510638297872 & 4.5698036112189E-016 & 0.0000000000000721560272766307 \\
\hline Ubl5 & 810 & 61.5501519756839 & 4.98414268966564E-016 & 0.0000000000000777021536277494 \\
\hline Tns3 & 340 & 25.8358662613982 & $6.21310483604413 \mathrm{E}-016$ & 0.0000000000000933178038545359 \\
\hline Gnas & 1105 & 83.9665653495441 & $8.09022339241375 \mathrm{E}-016$ & 0.000000000000120047218434901 \\
\hline Hmgn1 & 1071 & 81.3829787234043 & $8.28106180554255 \mathrm{E}-016$ & 0.000000000000121416139520312 \\
\hline Tmem176a & 1155 & 87.7659574468085 & $9.30796209618464 \mathrm{E}-016$ & 0.000000000000134866895501894 \\
\hline
\end{tabular}




\begin{tabular}{|c|c|c|c|c|}
\hline Itgav & 406 & 30.8510638297872 & $1.23611514030828 \mathrm{E}-015$ & 0.000000000000177023186837637 \\
\hline Park7 & 1019 & 77.4316109422492 & $1.51670376190678 \mathrm{E}-015$ & 0.000000000000211516259636277 \\
\hline Phip & 512 & 38.9057750759878 & 1.95567822990597E-015 & 0.000000000000267623700883577 \\
\hline Zbtb20 & 1134 & 86.1702127659575 & $3.01202663322541 \mathrm{E}-015$ & 0.000000000000398883010911873 \\
\hline Tia1 & 383 & 29.1033434650456 & $4.40807968443344 \mathrm{E}-015$ & 0.000000000000577552227590236 \\
\hline Edf1 & 994 & 75.531914893617 & 0.0000000000000121470419875807 & 0.00000000000151378699336682 \\
\hline Ambp & 1177 & 89.4376899696049 & 0.0000000000000121683105182945 & 0.00000000000151378699336682 \\
\hline Ppib & 1085 & 82.4468085106383 & 0.0000000000000133471343504952 & 0.00000000000164383306660699 \\
\hline Tmsb10 & 1139 & 86.5501519756839 & 0.000000000000017399819163864 & 0.00000000000210094287080538 \\
\hline $\operatorname{Tm} 4 \mathrm{sf} 4$ & 1257 & 95.516717325228 & 0.0000000000000206759587053386 & 0.00000000000247228259626166 \\
\hline Cst3 & 1140 & 86.6261398176292 & 0.0000000000000244009517551099 & 0.00000000000287166925463271 \\
\hline Rack1 & 1129 & 85.790273556231 & 0.0000000000000244824027067583 & 0.00000000000287166925463271 \\
\hline $\mathrm{H} 3 \mathrm{f3a}$ & 1158 & 87.9939209726444 & 0.0000000000000302081712786963 & 0.00000000000350091193403735 \\
\hline Rplp2 & 1107 & 84.1185410334347 & 0.0000000000000304155226487493 & 0.00000000000350091193403735 \\
\hline Hint1 & 1062 & 80.6990881458967 & 0.0000000000000325722449467062 & 0.00000000000371444230336698 \\
\hline Hsp90ab1 & 1243 & 94.4528875379939 & 0.0000000000000329568107766566 & 0.00000000000372381726170003 \\
\hline Ahnak & 454 & 34.4984802431611 & 0.0000000000000361240236362452 & 0.00000000000404457704639996 \\
\hline Esd & 866 & 65.8054711246201 & 0.000000000000042992116755536 & 0.00000000000477018837802867 \\
\hline Alb & 1237 & 93.9969604863222 & 0.0000000000000533275363098816 & 0.00000000000576124506309212 \\
\hline Prdx1 & 1176 & 89.3617021276596 & 0.0000000000000550453645654987 & 0.00000000000589511921729288 \\
\hline Scp2 & 948 & 72.0364741641337 & 0.0000000000000678746453288678 & 0.00000000000720641492991668 \\
\hline Nbeal1 & 458 & 34.8024316109422 & 0.000000000000106482357589854 & 0.0000000000111138704752258 \\
\hline $\operatorname{ltm} 2 b$ & 1204 & 91.4893617021277 & 0.000000000000109809295914124 & 0.0000000000113648007435156 \\
\hline Gabarap & 978 & 74.3161094224924 & 0.000000000000171153306958712 & 0.0000000000175660344041958 \\
\hline Tesc & 813 & 61.7781155015198 & 0.000000000000228189862389689 & 0.000000000023199144406452 \\
\hline Rps15a & 1143 & 86.854103343465 & 0.000000000000229806399609219 & 0.000000000023199144406452 \\
\hline Cystm1 & 819 & 62.2340425531915 & 0.000000000000277847080048863 & 0.0000000000278208507144861 \\
\hline Atp5a1 & 1022 & 77.6595744680851 & 0.000000000000298869170958368 & 0.000000000029684457334865 \\
\hline Mgst3 & 574 & 43.6170212765957 & 0.00000000000033594717616705 & 0.0000000000330637385195228 \\
\hline Uqcrh & 965 & 73.3282674772037 & 0.000000000000338261696448512 & 0.0000000000330637385195228 \\
\hline Atp5e & 907 & 68.9209726443769 & 0.000000000000413607235844545 & 0.0000000000401101316272553 \\
\hline Spcs1 & 960 & 72.9483282674772 & 0.000000000000427869995695632 & 0.0000000000409405729229211 \\
\hline Son & 805 & 61.1702127659575 & 0.000000000000432143104902545 & 0.0000000000409405729229211 \\
\hline Akr1a1 & 954 & 72.4924012158055 & 0.000000000000470313678377402 & 0.0000000000442166661289777 \\
\hline Gstt1 & 787 & 59.8024316109423 & 0.000000000000477629644427471 & 0.0000000000443156911359423 \\
\hline H2afv & 965 & 73.3282674772037 & 0.00000000000095513615713678 & 0.0000000000877869918753477 \\
\hline Gm609 & 510 & 38.7537993920973 & 0.00000000000112124703209021 & 0.000000000102290951460912 \\
\hline Gng5 & 1079 & 81.9908814589666 & 0.00000000000133235499026606 & 0.000000000119775796059247 \\
\hline Myl6 & 1170 & 88.9057750759879 & 0.00000000000183397692982801 & 0.000000000163675796143202 \\
\hline Selenbp1 & 682 & 51.8237082066869 & 0.00000000000189327518882542 & 0.000000000167752354140819 \\
\hline $\mathrm{B} 2 \mathrm{~m}$ & 1138 & 86.4741641337386 & 0.00000000000232533628988685 & 0.000000000201681984128496 \\
\hline Vps28 & 744 & 56.5349544072948 & 0.00000000000231200813012127 & 0.000000000201681984128496 \\
\hline A830018L16Rik & 224 & 17.0212765957447 & 0.00000000000362271435796281 & 0.000000000307705862294276 \\
\hline Naca & 1123 & 85.3343465045593 & 0.00000000000440331702141948 & 0.000000000371446934491797 \\
\hline $\mathrm{Cd} 24 \mathrm{a}$ & 1003 & 76.2158054711246 & 0.00000000000482931042720233 & 0.000000000404610797424652 \\
\hline Psma2 & 853 & 64.8176291793313 & 0.00000000000538666625747623 & 0.000000000448257983966738 \\
\hline Swi5 & 810 & 61.5501519756839 & 0.00000000000594324699257001 & 0.000000000486501171492006 \\
\hline Gstm5 & 694 & 52.7355623100304 & 0.00000000000596473505158274 & 0.000000000486501171492006 \\
\hline Etfb & 859 & 65.273556231003 & 0.00000000000611738031878882 & 0.000000000495668789514495 \\
\hline Psenen & 764 & 58.0547112462006 & 0.00000000000665385458286256 & 0.000000000535613549297617 \\
\hline
\end{tabular}

